Provided for non-commercial research and education use. Not for reproduction, distribution or commercial use.

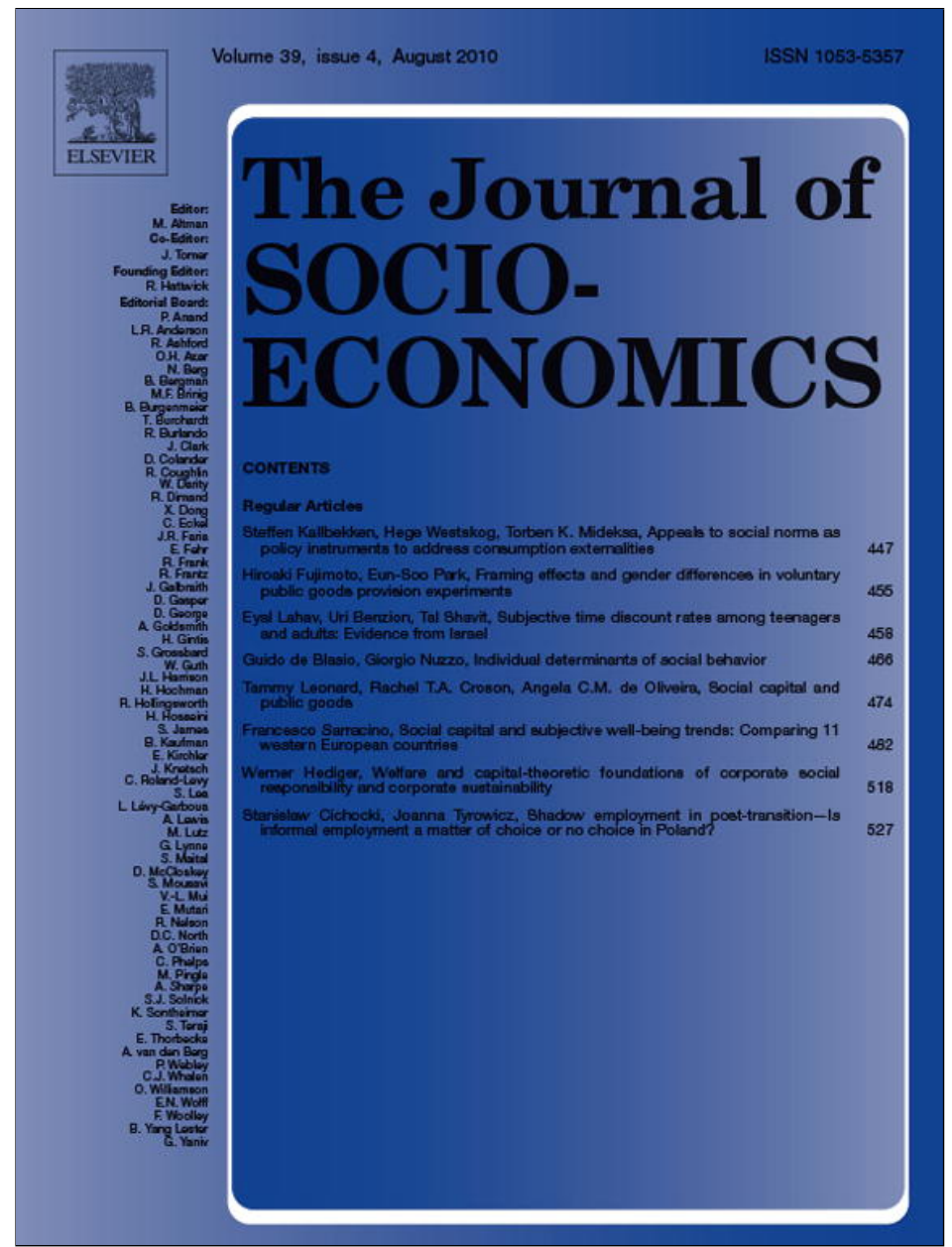

This article appeared in a journal published by Elsevier. The attached copy is furnished to the author for internal non-commercial research and education use, including for instruction at the authors institution and sharing with colleagues.

Other uses, including reproduction and distribution, or selling or licensing copies, or posting to personal, institutional or third party websites are prohibited.

In most cases authors are permitted to post their version of the article (e.g. in Word or Tex form) to their personal website or institutional repository. Authors requiring further information regarding Elsevier's archiving and manuscript policies are encouraged to visit:

http://www.elsevier.com/copyright 


\title{
Social capital and subjective well-being trends: Comparing 11 western European countries
}

\author{
Francesco Sarracino* \\ University of Firenze, Economic Sciences, via delle Pandette, 32, 50127 Firenze, Italy
}

\section{A R T I C L E I N F O}

\section{Article history:}

Received 29 April 2009

Received in revised form

18 September 2009

Accepted 26 October 2009

Social capital

Subjective well-being

Time-trends

Easterlin paradox

\begin{abstract}
A B S T R A C T
Discovering whether social capital endowments in modern societies have been subjected or not to a process of gradual erosion is one of the most debated topics in recent economic literature. This new stream of research has been inaugurated by Putnam's pioneering studies about social capital trends in the United States. Recently, a considerable work by Stevenson and Wolfers (2008) put a new emphasis on this topic contending Easterlin's assessment. Present work is aimed at analyzing the relationship between changes in social capital and subjective well-being in western Europe considering 11 different countries. In particular, I would like to answer questions such as: (1) is social capital in western Europe declining? Is such erosion a general trend of modern societies or is it a characteristic feature of only some of them? (2) social capital trend can help to explain subjective well-being trend? In so doing, my research considers four different set of proxies of social capital controlling for time and socio-demographic aspects in eleven different western European countries using World Values Survey (WVS) data between 1980 and 2000. My results are encouraging, showing evidence of a probable relationship between social capital and happiness. Furthermore, my results show that during last 20 years western European citizens have persistently lost confidence in the judicial system, in the church, in armed forces and the police. Finally, considering single countries, we discover that United Kingdom is the only country, among the investigated ones, with a negative pattern for social capital: the majority of the proxies of social capital in UK declined over the considered period.
\end{abstract}

(C) 2009 Elsevier Inc. All rights reserved.

\section{Introduction}

Discovering whether social capital (SC) endowments in modern societies have been subjected or not to a process of gradual erosion is one of the most debated topics in recent economic literature. This new stream of research has been inaugurated by Putnam's pioneering studies about SC trends in the United States. Considering numerous proxies of SC, Putnam (2000) argues that during last 30 years USA experienced a decline in social relationships and in its system of shared values and beliefs. From this point, much of the literature on SC tries to find evidence to support or to contend this statement. For a comprehensive review of such literature see Stolle and Hooghe (2004). Putnam's finding has been carefully scrutinised by Paxton (1999); Robinson and Jackson (2001); Costa and Kahn (2003), and Bartolini et al. (2008), while Ladd (1996) criticised this evidence. "On balance, social capital has been confirmed as declining in the US, although not so dramatically as Putnam claimed." All these studies are focused on the USA since similar research asks

\footnotetext{
* Corresponding aruthor.

E-mail address: f.sarracino@gmail.com.

1 See Bartolini et al. (2008).
}

for a generous database and the US General Social Survey (GSS) offers a long lasting temporal data-series. Consequently, we do not have much information about what happened in other countries in the same period Putnam (2002), Van Deth et al. (2000). For that reason the first question I would like to give an answer is: how is doing Europe? is SC declining? is such erosion a general trend of western societies or is it a characteristic feature of the American one? To my knowledge only a few authors payed attention to this aspect since only a few datasets are useful to establish a clear longterm pattern Arts and Halman (2004), Van OOrschot et al. (2006). In $2001 \mathrm{OECD}^{2}$ dedicated to this topic a publication in which, beyond others, dealt with the theme of trends in five European countries: United Kingdom, Netherlands, Sweden, France and Germany. The report assesses that in general SC declined, in particular in United Kingdom, while remaining countries show a more mixed pattern.

Another general perspective is offered by Leigh (2003). Contributing to an entry on "Trends in social capital" he identifies three common patterns of declining trust, political participation and organizational activity across industrialized countries in the

\footnotetext{
${ }^{2}$ See OECD (2001b), Centre for Educational Research and Innovation, Paris.
} 
period between 1980 and 1990. Among the five reviewed European countries (Britain, France, Germany, Spain and Sweden) only the Scandinavian one seems to have a positive trend even if civic engagement is declining. Further studies have been conducted by Norris (2004); Delhey and Newton (2005) but these studies focused on particular indexes of SC or only on generalised trust and were based on old data from the World Values Survey (WVS). A deeper analysis was conducted by Morales (2004) on trends and levels of associational participation in Europe. Looking at trends between 1980 and 2002 from the WVS and the European Social Survey (ESS) she concludes that it is not possible to state whether a clear increase or decrease in general levels of membership exists. Anyway, her analysis is merely descriptive and, even if she focuses on a broad set of countries, her conclusions do not account for other aspects, such as socio-demographic variables, that can affect SC trends. Finally, a more recent article by Adam (2008) observes trends of generalized trust and membership in voluntary organizations using data from WVS in the period 1980-2000. The author finds evidence of a non-eroding SC in Europe even if he warns about signs of decline as well as improvement. He states that decline in trust in individuals is quite visible, while associational involvement shows a more complex but on average positive trend.

Adam's work is, to my knowledge, the most up-to-date and complete research on European trends of SC. Anyway, it suffers some limitations. First of all it is based on mean variations between the starting and ending period. This is quite comprehensible since the second aim of the author was to test the reliability of the WVS vis-a-vis other databases (i.e. ESS), but in general this approach does not allow to check for other factors; secondly the author adopts only some of the available proxies of SC, namely generalized trust, membership in voluntary organizations and unpaid voluntary work; finally, Adam focuses on a large number of European countries including transition countries: this is an interesting point, but misses to account for different economic realities (developed and transition countries) preventing a more detailed knowledge of what happened to SC during last 20 years.

In order to overcome these limitations, my research considers four different set of proxies of SC controlling for time and socio-demographic aspects in eleven different western European countries. Data are drawn from the WVS, a dataset composed of four waves between 1980 and 2000. In so doing, I am able to investigate trends on a 20 years period.

The second question I would like to answer is whether SC trends can help to explain subjective well-being (SWB) trends. In a pioneering work Easterlin (1974) discovered that, using cross-section data, on average richer people are also happier than poorer ones; but a life-cycle analysis on the same sample shows that during time income grew up while happiness stayed constant. Such a puzzle is actually known as the "Easterlin paradox." Starting from this point an even more consistent part of the economic literature flourished trying to solve the problem Blanchower and Oswald (2004). Many different theories coming from manifold scientific fields have been advanced so far, but until now they failed to fully explain the paradox. ${ }^{3}$ Recently, Stevenson and Wolfers (2008) revive the debate challenging the existence of the paradox. Considering Europe and Japan they argue that societies get happier as they become richer. That is to say that "money can buy happiness." Unfortunately, at the same time they state that "the failure of happiness to rise in the United States remains a puzzling outlier." In this way the Easterlin paradox remains unsolved and also its nonexistence is not demonstrated. There is a need to further look into the "black box" of the American case. From this point of view, some recent contributions by Helliwell $(2001,2002,2006)$ propose SC as an important aspect for SWB arguing that money cannot explain the whole variation in people well-being. To my knowledge, the paper tackling most successfully with the challenge settled by Helliwell is Bartolini et al. (2008) $)^{5}$ which argues that SC, and in particular relational goods, is important for SWB. They do not deny the importance of income for happiness, but using data from the American GSS between 1975 and 2004 they find out that U.S. SWB is largely explained by four forces acting in different directions: (1) income growth; (2) decreasing relational goods; (3) decreasing confidence in institutions; (4) social comparisons. These four groups of variables allow to explain quite the whole variation in SWB. In other words, the three authors suggests that American happiness did not grow up together with economic growth because the positive effect of income growth was counterbalanced by the declining availability of SC which negatively affects SWB. In this way they provide a convincing and powerful explanation of the Easterlin paradox giving SC a new role: a higher income increases happiness as long as it does not undermine SC Bartolini and Bonatti (2003). Whenever this hypothesis would be corroborated by further research, policy agendas will have to consider also the effects of economic policy on the preservation and the provision of social capital. Hence, SC can become an important aspect of future development policies.

The theory proposed by Bartolini et al. (2008) can help to explain what happened in USA. A few example can probably be convincing. Estimates from the three authors suggest that in presence of a stable endowment of SC, and in particular of relational goods, American SWB would have been higher than the actual one. Similarly, if income growth should compensate for the effect of the reduction of SC on happiness, keeping this variable stable to its 1975 levels, then the growth rate of GDP should have been more than $10 \%$. Finally, they also estimate that the positive effect of income growth on SWB has been counterbalanced by the increase of other's people income (which offsets $2 / 3$ of the effect of income growth) and by the decrease in relational goods and confidence in institutions (which accounts for $5 / 6$ of the total effect of social comparisons on SWB).

Concluding, the contribution by Bartolini et al. (2008) seems to suggest that differences in SC trends can help to explain differences in SWB trends. The aim of present work is to provide further evidence to support this hypothesis looking at some European countries. Main results of my research are the following:

1. SC trends in the majority of the western European countries are different from the American ones. Great Britain is the country with the worst trend, among the investigated ones, for SC.

2. SWB trends in present sample of countries are generally positive with the only exception of Great Britain.

3. SC and SWB trends for investigated European countries are compatible with a relational explanation of the Easterlin paradox.

Present work is structured in four sections: the first section outlined my research questions and motivations behind them; the second section points out data adopted for my research and methodological aspects; the following section reports results from different regressions considering various proxies of SC as dependent variable and adopting time dummies and socio-economic conditions as independent variables. Finally, some concluding remarks will follow.

\footnotetext{
${ }^{3}$ For a review of the main theories advanced so far please refer to Sarracino (2008).

${ }^{4}$ See Stevenson and Wolfers (2008), p. 16.
} 


\section{Data and methodological aspects}

The analysis of SC trends for different European countries asks for a generous dataset. From this point of view, probably, the most comprehensive database is represented by WVS. It is a wide compilation of surveys collected in more than 80 countries representing more than $80 \%$ of the world's population. It collects information on sociocultural and political change observed on a randomly selected sample of 300 to 4000 individuals per country. In particular the database provides information on "individual beliefs about politics, the economy, religious, social and ethical topics, personal finances, familial and social relationships, happiness and life satisfaction." 6 Data have been collected in four waves (1980-1982; 1990-1991; 1995-1997 and 1999-2001) for a total of 267,870 observations covering quite a long period of time-about 20 years. Anyway, the sample available for present study is smaller since I focus on the trend of SC indicators in a small subset of countries for which I have enough observations during time. Furthermore, since my aim is to check whether different economic systems have different performances comparing Western Europe and USA, I also exclude all those countries that have been subjected to any recent institutional shock. ${ }^{7}$ Considered countries are: Italy, France, the Netherlands, Belgium, United Kingdom, Ireland, Germany, Denmark, Sweden, Norway and Finland.

Although SC has been longly a much debated topic, actually it still lacks a commonly agreed definition Durlauf and Fafchamps, 2004, Tinggaard Svendsen et al., 2009. This topic has been developed and applied in many different social disciplines hence different definitions have been advanced so far. Some of the fathers of this concept propose different definitions for it. For example, Pierre Bourdieu, probably the first scientist introducing this term, defines social capital as "the aggregate of the actual or potential resources which are linked to possession of a durable network of more or less institutionalized relationships of mutual acquaintance and recognition ... which provides each of its members with the backing of collectively-owned capital." ${ }^{8}$ Such a definition focuses on three important aspects of social capital: (1) the existence of a network of individuals; (2) participation in this network; and (3) social capital as a public good. Nonetheless, Bourdieu misses to precisely identify social capital pointing on its sources: "the network of relationships." Differently, James Coleman proposes the following definition: "social capital is the set of resources that inhere in family relations and in community social organization and that are useful for the cognitive or social development of a child or a young person." 9 In Coleman's view the network aspect is less emphasized while he stresses the importance of the group in which social relations constitute useful capital resources. Such a concept can be related to the category of "bonding" social capital in contrast with that one of "bridging" social capital. Bonding refers typically to "relations among members of families and ethnic groups. Bridging social capital refers to relations with distant friends, associates and colleagues." ${ }^{10}$ These are two different forms of social capital that should be considered mutual. In fact, while the first form gives particular groups of people "a sense of identity and common purpose, without bridging ties that transcend various social divides (e.g. religion, ethnicity, socio-economic status), bonding ties can become a basis for the pursuit of narrow interests, and can actively exclude outsiders." 10 Such groups can

\footnotetext{
6 Bruni and Stanca (2006), p. 6.

7 Countries excluded from the sample are Spain, Portugal, Greece and Luxembourg.

${ }^{8}$ Quoted in Schuller et al. (2000), p. 5.

9 Quoted in Schuller et al. (2000), p. 6.

10 See OECD (2001b), p. 42.
}

be characterized by strong and co-operative norms, but low trust and co-operation with the rest of society becoming a barrier to social cohesion and personal development. Taking this aspect to the extreme, strong group ties can bring to neglect wider "public" interests promoting socially destructive "rent-seeking" activities (Olson, 1982). Finally, Robert Putnam defines social capital the "features of social life-networks, norms, and trust-that enable participants to act together more effectively to pursue shared objectives." 11 In this way the author identifies crucial aspects of social capital specifying their role in social relationships: they enable different people to co-operate (even unconsciously) to reach common goals Putnam (2001). Nonetheless, given the empirical nature of present work, I opted for a more operating definition such as the one proposed by Bartolini et al. (2008) who define SC as "the stock of both non-market relations and beliefs concerning institutions that affect either utility or production functions." ${ }^{12}$ In this way the authors do not focus solely on particular aspects of SC-networks, norms and trust-but comprise all those aspects-material and immaterial-that can contribute to develop mutual trust and co-operation. In particular, they point to two main aspects of SC: (1) every non-market relationships among individuals which allow people to communicate each other and to develop mutual trust. They define this aspect relational SC; (2) the system of values or believes that makes people act coherently. Moreover, the authors propose a further distinction in intrinsically and extrinsically motivated relational SC depending on whether the incentives to act come from within or outside the individual. They define intrinsic SC (alternatively defined as relational goods) those components "that enter into people's utility function"13; by extrinsic SC they mean those components that do not "directly enter into people's utility functions but are instrumental to something else that may be considered valuable." This distinction allows to go deeper in the analysis of the category of relational SC. In fact, quoting Deci's work (1971), they focus on the non-instrumental nature of intrinsic motivated activities. This peculiarity allows to focus on a broader point: non-market relations are not always intrinsic; there can be extrinsic relational SC (or purely extrinsic) as well as intrinsic one. ${ }^{14}$

A further critical aspect about SC is how to measure it Durlauf (2002). Different proposals have been advanced, but generally there are some agreed proxies of SC. For example, following Putnam (2000) main measures of SC centre around proxies of trust and levels of engagement or interaction in social or group activities. When trying to measure SC we should keep in mind particular aspects (OECD, 2001a):

- we should pay attention to causal connections since sources, functions and outcomes may be confused;

- SC is mainly characterized by tacit and relational aspects which are naturally difficult to observe, to measure and to codify;

- usual variables of SC (trust, membership, voting, etc.) provide proxy measures and should not be confused with the underlying concept.

According to the vast majority of the literature on SC (Paxton, 1999; Costa and Kahn, 2003; Van and Schaik, 2002), I observe the beliefs component through several reports of confidence in institutions, namely armed forces, police, parliament, civil services, press, ecclesiastic, judicial system, education system, labour unions and major companies. Answers to these questions range on a 1 to 4 point scale going from none at all to a great deal. To measure

\footnotetext{
11 Putnam (1993), p. 56.

12 See Bartolini et al. (2008), p. 5

13 See Bartolini et al. (2008), pp. 5-6

14 Please refer to Table A.1 in Appendix A for a summarizing scheme.
} 
non-market relations, I use trust in individuals (represented by a dummy variable), membership and unpaid voluntary work in various groups and organizations. Given the multiple nature of the last two proxies, I adopt the mentioned distinction between intrinsically and extrinsically motivated group participation (Bartolini et al., 2008). Groups and organizations entering the first set are labelled Putnam's groups while those comprised in the second one are named Olson's group (Knack, 2003). This distinction is based on the works of the two authors: Olson (1982) emphasizes the tendency of associations to act as lobbies to get policies that protect the interest of special groups at the expenses of the society as a whole. Consequently, I include in Olson's groups all those groups and organizations which are extrinsically motivated since it is supposed they are experienced only for instrumental reasons. On the contrary, Putnam (1993) identifies in associations a source of general trust and of social ties leading to governmental and economic efficiency (Bartolini et al., 2008). In this paper putnamian groups are interpreted as intrinsic SC supposing they are experienced only for the pleasure of being a member. Among Putnam's group I include social welfare service for elderly, church organizations, sport clubs, art and literature clubs, fraternal groups and youth associations, human and animal rights, peace movements and environmental groups. Among Olson's groups I include fraternity associations, unions, professional organizations and farm organizations, organization concerned with health and consumer groups. Finally, there are some groups that were left unclassified and labeled as other groups because it is not clear whether they constitute intrinsic or extrinsic RSC, although they are part of RSC. In this latter group I included veterans associations, political parties and "other groups." Each option between these three groups of variables is expressed as a dummy variable.

Finally, SWB is proxied by the variable happiness that is measured on a scale ranging from 1 to 4 and is based on answers to the following question: "All considered you would say that you are: 1. very happy; 2. pretty happy; 3. not too happy; 4. not at all happy?".

In order to study SC and SWB trends during the last 20 years for each of the considered European countries, I follow two approaches ${ }^{15}$ : I first regress the proxies of SC and SWB on time dummy variables. In this way trends are based on mean values; than I regress the same proxies on different groups of control variables (age, gender, familiar status and education) to check whether such trends depend on peculiar individual and social aspects. In particular, age is considered linearly and with its square; a dummy on male is introduced; familiar status is controlled through three proxies: the number of children, a variable ranging between zero and twenty, and two dummy variables for single and married; finally, education includes a dummy for illiterate.

This model is repeated for each considered country. Formally, I estimate the following:

$$
\begin{aligned}
\text { Proxy }_{i t}^{j}= & \alpha+\beta_{1} \cdot D_{i, w_{2}}+\beta_{2} \cdot D_{i, w_{3}}+\beta_{3} \cdot D_{i, w_{4}}+\gamma_{1} \cdot \text { Age }_{i t} \\
& +\gamma_{2} \cdot \text { Age }_{i t}^{2}+\gamma_{3} \cdot \text { Male }_{i}+v_{1} \cdot \text { NChild }_{i t}+v_{2} \cdot \text { Single }_{i t} \\
& +v_{3} \cdot \text { Married }_{i t}+\delta_{1} \cdot \text { Illiterate }_{i t}
\end{aligned}
$$

where index $j$ stands for the different proxies of SC and SWB, index $t$ represents the various waves and index $i$ stands for each individual. In each equation three dummy variables have been introduced to account for the four waves. Where possible I kept the first wave as the reference period. When information about the first waves where not available, I adopted the second wave as reference period.

Since I have different indicators of SC and one proxy of SWB, my regression methodology varies following the specifities of each depending variable: in the case of generalized trust, participa-

\footnotetext{
15 See Aguiar and Hurst (2006).
}

tion in voluntary organizations and unpaid voluntary work, that are expressed in the form of dummies, I adopted a logit model; when studying confidence in institutions or happiness, which are ordered variables, I used an ordered logit model. Tables A.2-A.12 in Appendix A report summary statistics for each considered country.

When dealing with these data we have to be careful because, although the WVS is the most complete database on our topic, it has some deficiencies. In particular, we have to keep in mind that observations about Italy, Ireland, Denmark, France, The Netherlands and Belgium are missing in the third wave; similarly, data about Finland are not collected in the first wave, while Norway is not observed in the fourth wave. Finally, the third wave does not contain information about trust in the United Kingdom and about confidence in the educational system in Sweden, Norway, Finland and Germany. Overall, the pooled dataset contains 48,340 observations.

\section{Results}

\subsection{Social capital trends in western Europe}

I report and discuss results from several regressions relative to Eq. (1). Results about each regression are reported in Appendix A in Tables A.13-A.23. Here I discuss directly my conclusive results which are summarized in charts in Appendix B.

A first interesting aspect emerging from my regressions is that SC trend in considered European countries is mainly positive. Hence, the picture about western Europe appears different from the American one. There is only one country that seems more similar to USA, the Great Britain. In this case the majority of the considered proxies of SC is declining meaning that during last 20 years Great Britain experienced an erosion of SC. Charts from Figs. B. 1 to B.7 show this result. On the $x$-axis I report the time from 1980 to 2000. Each point on the $x$-axis corresponds to a wave in the WVS. On the $y$-axis I report coefficients of the time dummies originating from regressions. The point on the $x$-axis corresponding to zero represents the reference year, while other points in the charts defining trends corresponds to the coefficients of the time dummies. Finally, each chart reports more than one line. Each line represents results from regressions with different sets of control variables, coherently with the adopted model. Charts suggest that in Great Britain SC, and in particular membership in groups or organizations and trust in others, decreases strongly during all the considered period. Similarly, every proxy of beliefs in institutions declines steadily all along the last 20 years. This picture changes if we turn considering unpaid voluntary work. Figs. B.1(c) and B.2(c) and (d) suggest that all these proxies have been increasing during last 20 years in stark contrast with the other proxies of relational SC.

Overall, the evolution in time of British SC seems to be similar to the American one for what concern trust, membership in groups and associations and trust in others, while a more optimistic conclusions may be drawn considering unpaid voluntary work.

The picture is completely different if we consider remaining countries. First of all, the strong contradiction between membership and unpaid voluntary work observed for Great Britain disappears: looking at charts from Figs. B.8(c) to B.14(c) we observe that in all these cases the trends of the two proxies are concordant. Secondly, trends about relational goods are generally positive. Here I will discuss only results for some of the major countries of the sample. Considering membership in Putnam's groups, charts from Figs. B.8(a) to B.10(a) suggest that Italy, the Netherlands and Sweden from 1980 to 2000 experienced a growing trend. Figs. B.11(a) and B.12(a) show that the same trend is positive also in France and in Denmark, even if in these two cases relative growth rate reduces since 1990. Considering Norway, Fig. B.13(a) suggests a positive trend, but in this case available data do not allow to set a clear 
pattern. I can only conclude that in this case the trend between 1980 and 1990 is positive. Finally, the chart about Germany ${ }^{16}$ (Fig. B.14(a)) points out that overall from 1980 to 2000 membership in Putnam's groups is positive, but I have to remark that the trend reverted since 1990 .

Considering the other component of relational goods, that is to say trust in others, the picture emerging from regressions is more homogeneous, since it grows up in every of the mentioned countries. I have only to highlight two cases: (1) Italy, in which the overall trend is positive although the growth rate of trust in other's slightly reduces starting from 1990; (2) France, which emerges as the only Continental European country, among the investigated ones, with a decreasing trend of trust in others during last 20 years (please, consider (b) charts from Figs. B.8 to B.14.

Let's turn now to the second component of SC: beliefs in institutions. In this case trends are more mixed among both variables and countries. In any case, some general trends arise quite clearly indicating a worrying trend for confidence in some institutions: in particular, it seems that during last 20 years European citizens have persistently lost confidence in the judicial system, in religious institutions, in armed forces and in police.

Overall, we can state that, although some specificities and a mixed pattern regarding confidence in institutions, results suggest that the evolution of SC during time in the considered European countries is different from the American one. In this framework, the experience of Great Britain appears as peculiar and, at least regarding the majority of the considered proxies, more similar to the American one.

\subsection{Social capital and subjective well-being in western Europe}

Previous results conveyed a framework in which western European countries appear as very different from the USA. For quite every considered country, relational SC increased from 1980 to 2000. Regressions about the trend of SWB in the same countries confirm a similar pattern. In fact, SWB increases in every considered country with the exception of Great Britain in which SWB is strongly decreasing between 1980 and 1995. Unfortunately, data about the fourth wave are not available in this case (see Figs. B.15 to B.19 in Appendix B). Charts about remaining considered European countries show an overall positive pattern, even if single trends may differ. For example, France, Norway, Denmark and Netherlands have a steady growing trend (see Figs. B.17(b), B.18(a) and (b), and B.16(b)); trends for Germany and Italy are positive too, but the growth rate reduces significantly between 1990 and 2000 (see Figs. B.16(a) and B.19 in Appendix B); finally, Sweden's trend has a U-shaped outline (see Fig. B.17(a)), even if the net result is positive.

\section{Conclusions}

The aim of present study was to point out trends of social capital in western European countries finding evidence to support the thesis that SC trends can help to explain SWB trends. In this way SC gains a new dimension: it can give further meaning to the widely used term well-being. Whenever present thesis would be corroborated by further research, SC would acquire a central role in the definition of our policy agenda. For example, future economic policies should not only focus on ways to promote economic growth, but should pay attention also to their effects on SC.

Using different regression techniques, following the nature of dependent variables, I tried to assess the trends of four proxies of
SC for each country in the period between 1980 and 2000. Following a broadly accepted approach in the literature, I adopted the following variables: trust in individuals, membership in eighteen different voluntary organizations, performing unpaid voluntary work in 18 organizations and confidence in ten institutions. Results are quite innovative for at least two reasons: (1) contemporary literature largely focused on trends in USA rather than in European countries. This is mainly due to the fact that USA have large databases allowing such studies for longer periods of time (for example the U.S. GSS); (2) following the debate on the Easterlin paradox, my results suggest that we cannot discard the hypothesis that the trend of SC is important for the trend of SWB. From this point of view, it is important to stress that I am not performing a causal analysis, but I am simply assessing SC and SWB trends and notice that in 10 out of 11 countries signs of SC trends are concordant with signs of SWB trends. Such finding implies also that the theoretical predictions of the NEG model are largely met confirming the relevance of the model as explanatory tool. Moreover, whether such evidence would be substantiated by future research, we could say that USA do not represent a "puzzling outlier" since "income growth is desirable as far as it is not associated with a deterioration of SC." 17 Nonetheless, the question about whether SC trend can help to explain SWB trend is still an open question asking for further and deeper research.

Summarizing, my findings are the following:

1. Trends for SC in the analysed European countries are mainly positive (in particular for relational goods).

2. Although the trends of membership and unpaid voluntary work in Great Britain are contrasting, still this country appears as an exception in the European landscape with declining trends for the majority of the SC proxies.

3. All the considered countries seem affected by a general crisis of some particular institutions.

4. Given the concordance between SC and SWB trends in 10 out of 11 cases, we cannot reject the hypothesis that SC can help to explain SWB.

Concluding, present research allows to remark a few aspects: the first one is that the majority of the western European countries and USA are not exactly following the same pattern. While both regions have experienced an institutional crisis during last 20 years, relational social capital and subjective well-being in western Europe increased. Nonetheless, we should take in mind that these figures need further investigation to extend both the number of observed countries and the length of the considered period. By now, present results suggesting a quite different pattern between USA and the western European sample push future research in two main directions: (1) to enlarge present research to discover trends relative to other countries; (2) to investigate the causes of such a different performance. Which forces have pushed toward an increasing erosion of social capital in USA? Is European social capital subjected to the same erosive forces? (3) Do SC trends explain SWB trends in Europe?

\section{Acknowledgements}

The author is really grateful to Stefano Bartolini and Ennio Bilancini for their precious comments and advices on every step of present work. Special thanks also to Malgorzata Mikucka for her support and useful hints. The usual disclaimers apply. 


\section{Appendix A.}

Tables A.1-A.23

Table A.1

Summarizing scheme of the different constituents of social capital.

\begin{tabular}{|c|c|c|}
\hline \multirow{3}{*}{ 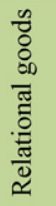 } & \multicolumn{2}{|c|}{ trust in others } \\
\hline & \multicolumn{2}{|c|}{ membership in putnamian groups } \\
\hline & \multicolumn{2}{|c|}{ Unpaid voluntary work in putnamian groups } \\
\hline \multirow{2}{*}{ 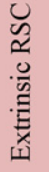 } & \multicolumn{2}{|c|}{ membership in olsonian groups } \\
\hline & \multicolumn{2}{|c|}{ Unpaid voluntary work in olsonian groups } \\
\hline \multirow{2}{*}{ 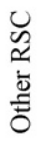 } & \multicolumn{2}{|c|}{ membership in other groups } \\
\hline & \multicolumn{2}{|c|}{ unpaid voluntary work in other groups } \\
\hline \multirow{10}{*}{$\begin{array}{l}u \\
\widetilde{Z} \\
\dot{1} \\
\tilde{0} \\
z\end{array}$} & \multirow{10}{*}{$\begin{array}{l}\Xi \\
0 \\
0 \\
0 \\
0 \\
0 \\
0 \\
0 \\
0\end{array}$} & Church \\
\hline & & Armed forces \\
\hline & & Educational System \\
\hline & & Press \\
\hline & & Labor Unions \\
\hline & & Police \\
\hline & & Parliament \\
\hline & & Civil services \\
\hline & & Major Companies \\
\hline & & Judicial System \\
\hline
\end{tabular}

Table A.2

Descriptive statistics about Italy.

\begin{tabular}{|c|c|c|c|c|c|c|c|c|c|c|c|c|c|c|c|c|}
\hline \multirow{2}{*}{$\begin{array}{l}\text { Italy } \\
\text { Variable }\end{array}$} & \multicolumn{5}{|c|}{ Wave 1} & \multicolumn{5}{|c|}{ Wave 2} & \multirow{2}{*}{$\begin{array}{l}\text { Wave } 3 \\
\text { Obs }\end{array}$} & \multicolumn{5}{|c|}{ Wave 4} \\
\hline & Obs & Mean & Std. Dev. & Min & Max & Obs & Mean & Std. Dev. & Min & Max & & Obs & Mean & Std. Dev. & Min & Max \\
\hline Happiness & 1324 & 2.879 & 0.632 & 21 & 4 & 1971 & 2.990 & 0.602 & 1 & 4 & 0 & 1975 & 2.952 & 0.693 & 1 & 4 \\
\hline Trust in others & 1302 & 0.268 & 0.443 & 0 & 1 & 1932 & 0.353 & 0.478 & 0 & 1 & 0 & 1946 & 0.326 & 0.469 & 0 & 1 \\
\hline Putnam's group & 1348 & 0.127 & 0.333 & 30 & 1 & 2018 & 0.246 & 0.431 & 0 & 1 & 0 & 2000 & 0.314 & 0.464 & 0 & 1 \\
\hline Olson's group & 1348 & 0.103 & 0.304 & 40 & 1 & 2018 & 0.123 & 0.328 & 0 & 1 & 0 & 2000 & 0.171 & 0.377 & 0 & 1 \\
\hline Other groups & 1348 & 0.084 & 0.277 & $7 \quad 0$ & 1 & 2018 & 0.108 & 0.310 & 0 & 1 & 0 & 2000 & 0.108 & 0.310 & 0 & 1 \\
\hline Unpaid work in putnamian groups & 1348 & 0.103 & 0.304 & 40 & 1 & 2018 & 0.184 & 0.388 & 0 & 1 & 0 & 2000 & 0.212 & 0.408 & 0 & 1 \\
\hline Unpaid work in olsonian groups & 1348 & 0.062 & 0.240 & 0 & 1 & 2018 & 0.065 & 0.247 & 0 & 1 & 0 & 2000 & 0.074 & 0.262 & 0 & 1 \\
\hline Unpaid work in other groups & 1348 & 0.045 & 0.206 & 0 & 1 & 2018 & 0.060 & 0.237 & 0 & 1 & 0 & 2000 & 0.054 & 0.226 & 0 & 1 \\
\hline \multicolumn{17}{|l|}{ Confidence in } \\
\hline Church & 1348 & 2.628 & 1.065 & 51 & 4 & 2016 & 2.724 & 0.991 & 1 & 4 & 0 & 1975 & 2.870 & 0.891 & 1 & 4 \\
\hline Armed forces & 1348 & 2.542 & 0.954 & 1 & 4 & 2012 & 2.352 & 0.859 & 1 & 4 & 0 & 1948 & 2.524 & 0.825 & 1 & 4 \\
\hline Educational system & 1348 & 2.568 & 0.872 & 21 & 4 & 2017 & 2.453 & 0.813 & 1 & 4 & 0 & 1966 & 2.596 & 0.816 & 1 & 4 \\
\hline Press & 1348 & 2.131 & 0.814 & 1 & 4 & 2013 & 2.281 & 0.778 & 1 & 4 & 0 & 1954 & 2.271 & 0.754 & 1 & 4 \\
\hline Labour Unions & 1348 & 2.020 & 0.858 & 1 & 4 & 2009 & 2.156 & 0.809 & 1 & 4 & 0 & 1927 & 2.090 & 0.804 & 1 & 4 \\
\hline Police & 1348 & 2.708 & 0.879 & 1 & 4 & 2012 & 2.701 & 0.746 & 1 & 4 & 0 & 1968 & 2.767 & 0.748 & 1 & 4 \\
\hline Parliament & 1348 & 2.082 & 0.847 & 1 & 4 & 2011 & 2.122 & 0.803 & 1 & 4 & 0 & 1944 & 2.222 & 0.780 & 1 & 4 \\
\hline Civil Services & 1348 & 2.022 & 0.827 & 1 & 4 & 2013 & 2.002 & 0.801 & 1 & 4 & 0 & 1944 & 2.216 & 0.738 & 1 & 4 \\
\hline Major Companies & 1348 & 2.073 & 0.880 & 1 & 4 & 2005 & 2.631 & 0.807 & 1 & 4 & 0 & 1879 & 2.444 & 0.779 & 1 & 4 \\
\hline Judicial system & 1348 & 2.372 & 0.880 & 1 & 4 & 2012 & 2.153 & 0.821 & 1 & 4 & 0 & 1946 & 2.184 & 0.808 & 1 & 4 \\
\hline Age & 1348 & 39.553 & 16.872 & 17 & 86 & 2018 & 41.353 & 16.094 & 18 & 88 & 0 & 2000 & 45.284 & 16.888 & 18 & 92 \\
\hline Age2 & 1348 & 1848.942 & 1478.435 & 289 & 7396 & 2018 & 1968.936 & 1455.248 & 324 & 7744 & 0 & 2000 & 2335.641 & 1617.433 & 324 & 8464 \\
\hline Male & 1348 & 0.493 & 0.500 & 0 & 1 & 2018 & 0.478 & 0.500 & 0 & 1 & 0 & 2000 & 0.480 & 0.500 & 0 & 1 \\
\hline No. of children & 766 & 2.275 & 1.311 & 1 & 8 & 1983 & 1.317 & 1.337 & 0 & 6 & 0 & 1850 & 1.402 & 1.330 & 0 & 9 \\
\hline Single & 1348 & 0.355 & 0.479 & 0 & 1 & 2018 & 0.315 & 0.464 & 0 & 1 & 0 & 2000 & 0.309 & 0.462 & 0 & 1 \\
\hline Married & 1348 & 0.564 & 0.496 & 0 & 1 & 2018 & 0.581 & 0.493 & 0 & 1 & 0 & 2000 & 0.584 & 0.493 & 0 & 1 \\
\hline Illiterate & 1348 & 0 & 0 & 0 & 0 & 2018 & 0 & 0 & 0 & 0 & 0 & 2000 & 0.065 & 0.247 & 0 & 1 \\
\hline
\end{tabular}




\section{Author's personal copy}

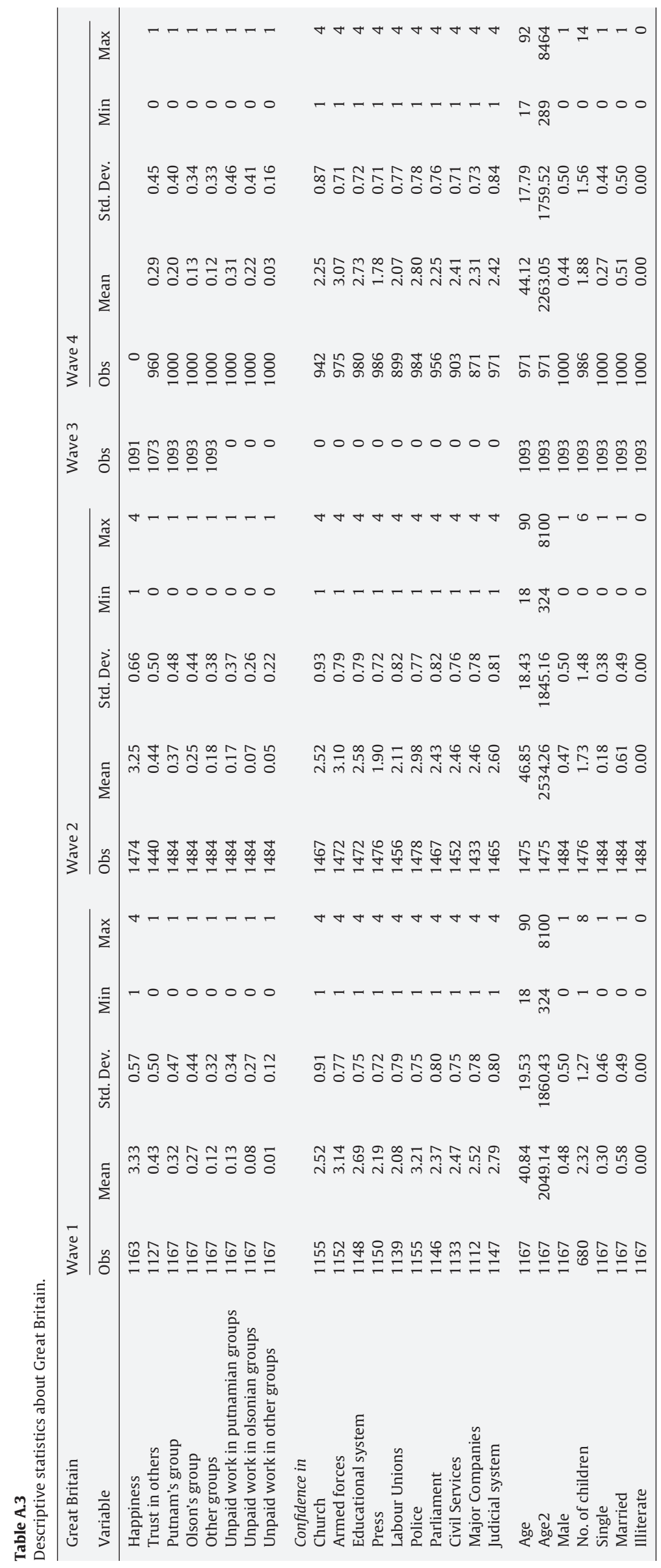




\section{Author's personal copy}

F. Sarracino / The Journal of Socio-Economics 39 (2010) 482-517

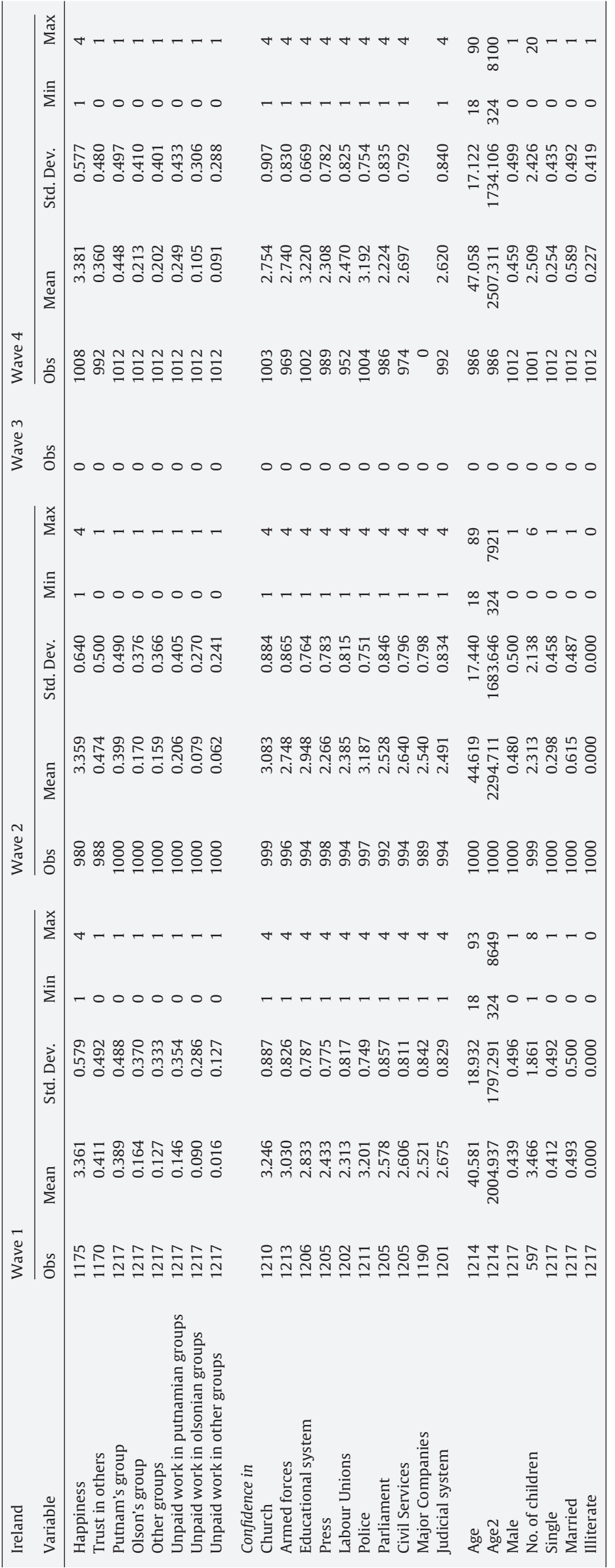




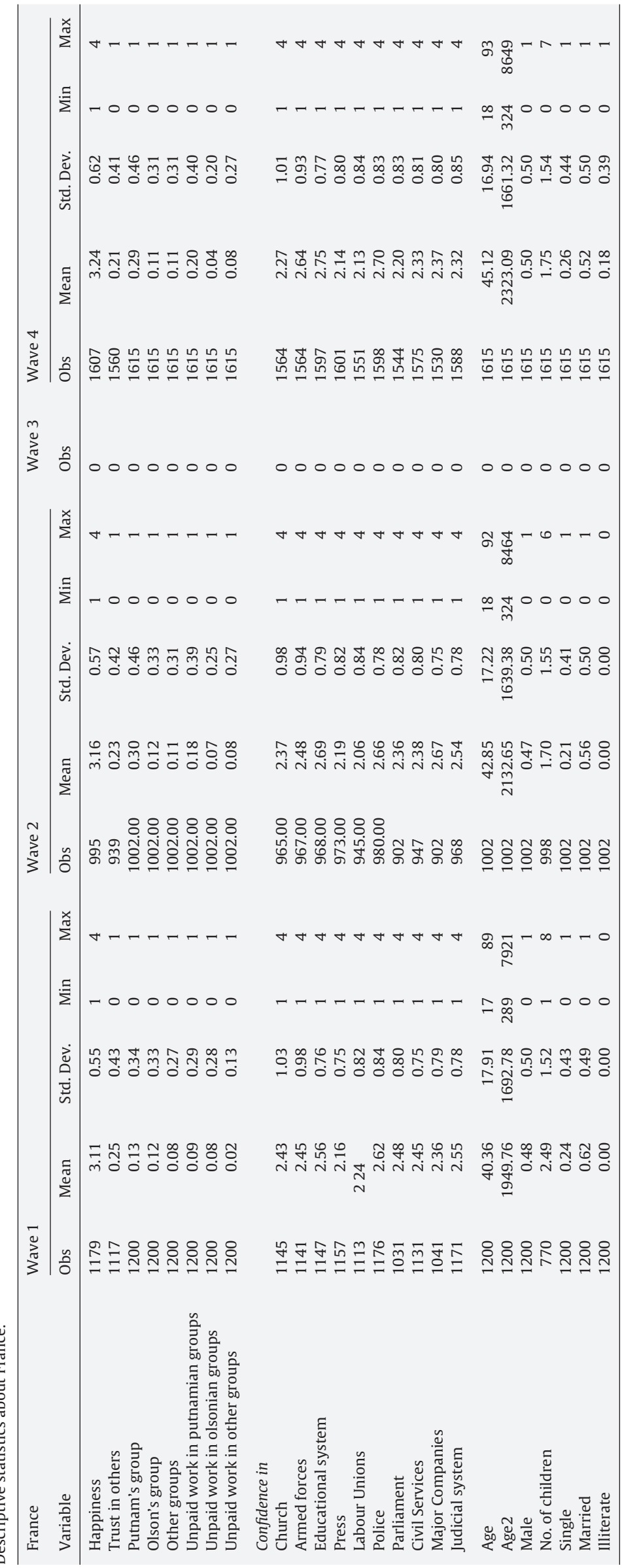




\section{Author's personal copy}

F. Sarracino / The Journal of Socio-Economics 39 (2010) 482-517

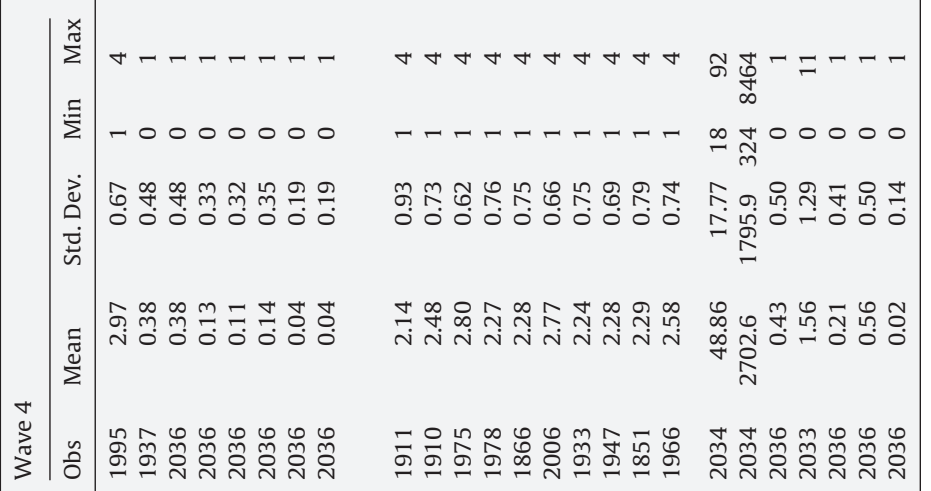

৪

$-0000$

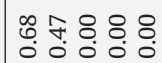

m

กิ่

获

ชิ

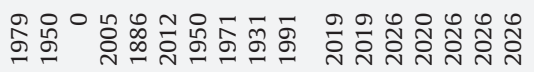

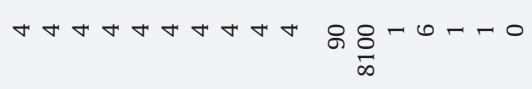

T-T-U-7-T

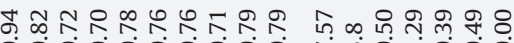

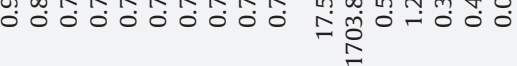

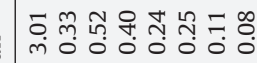

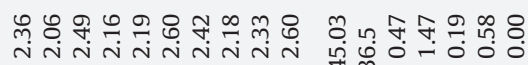

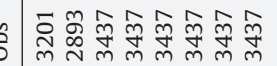

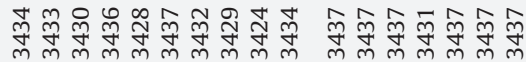

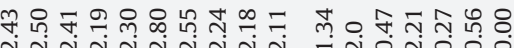
ㄱำ 


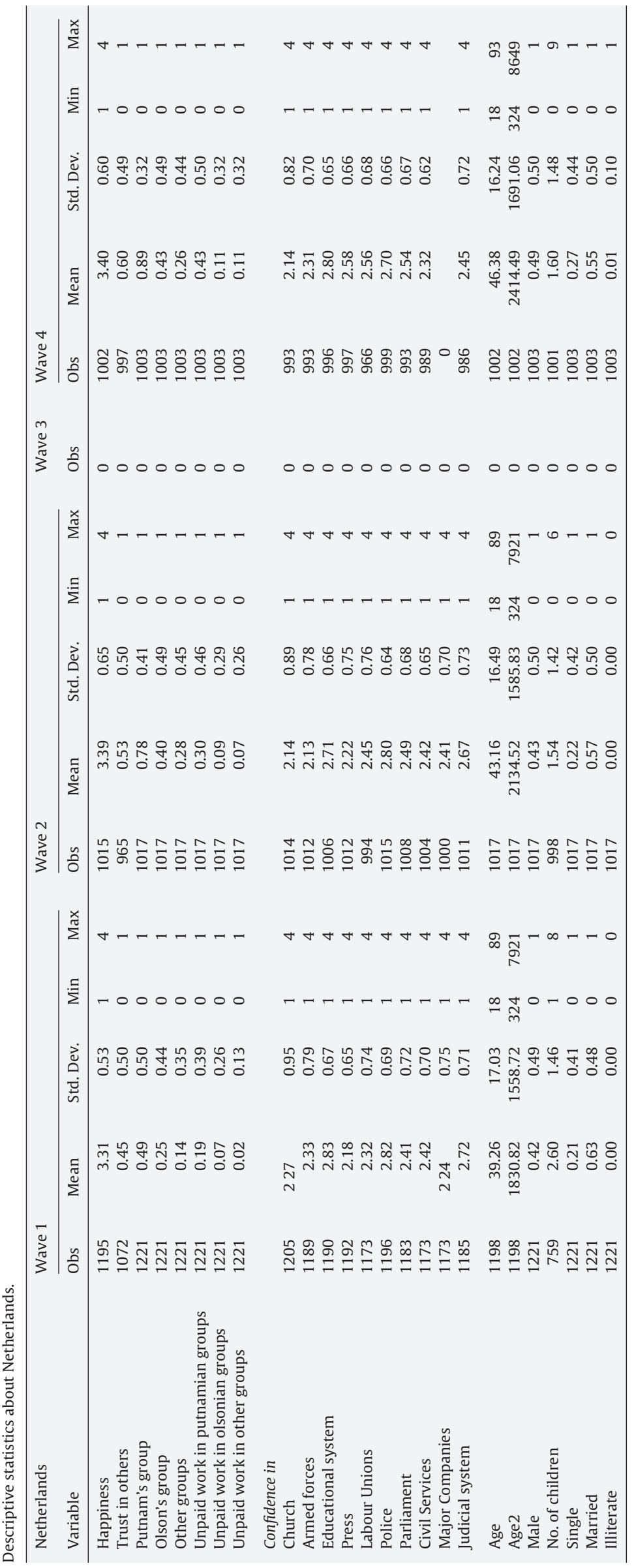




\section{Author's personal copy}

F. Sarracino / The Journal of Socio-Economics 39 (2010) 482-517

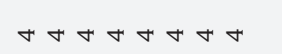

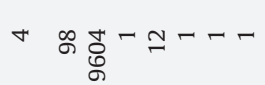

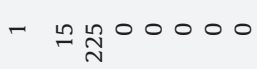

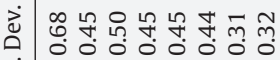

$r-r-r-r$

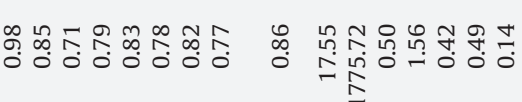

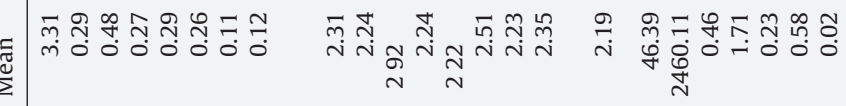

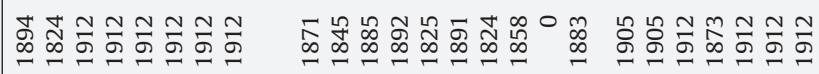

०ิ 00000000

00000000000000000

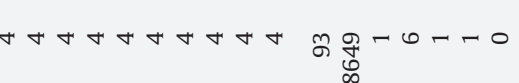

$-0000000$

- - - -

仓ัँ

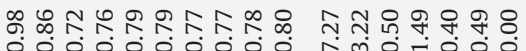
䓀

ॠ్ల

市

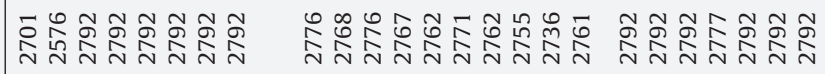

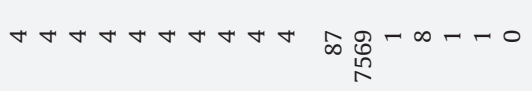

$\sum-0000000$

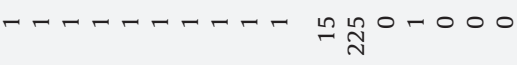

仓ั๊

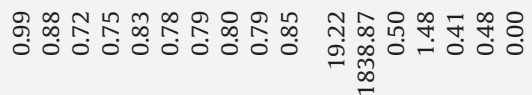

范

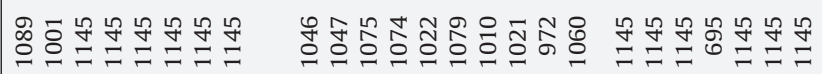




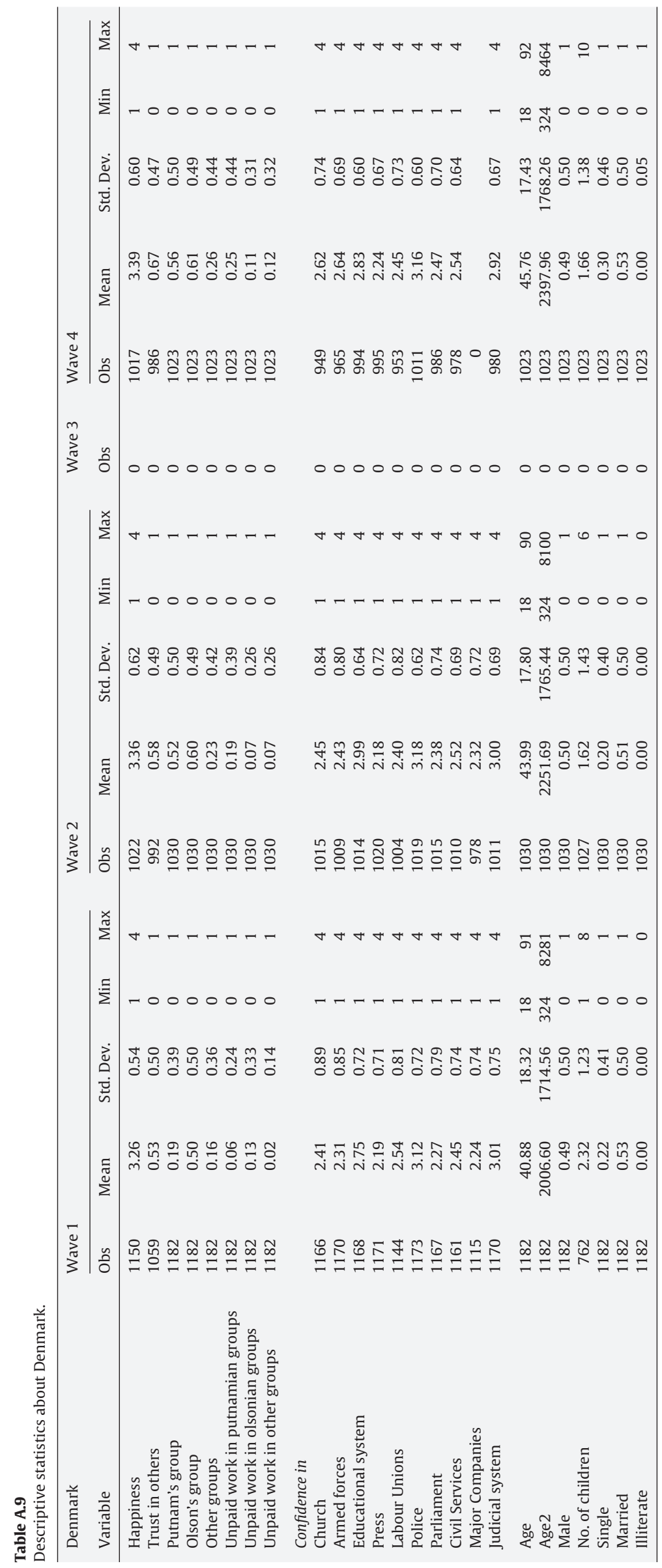




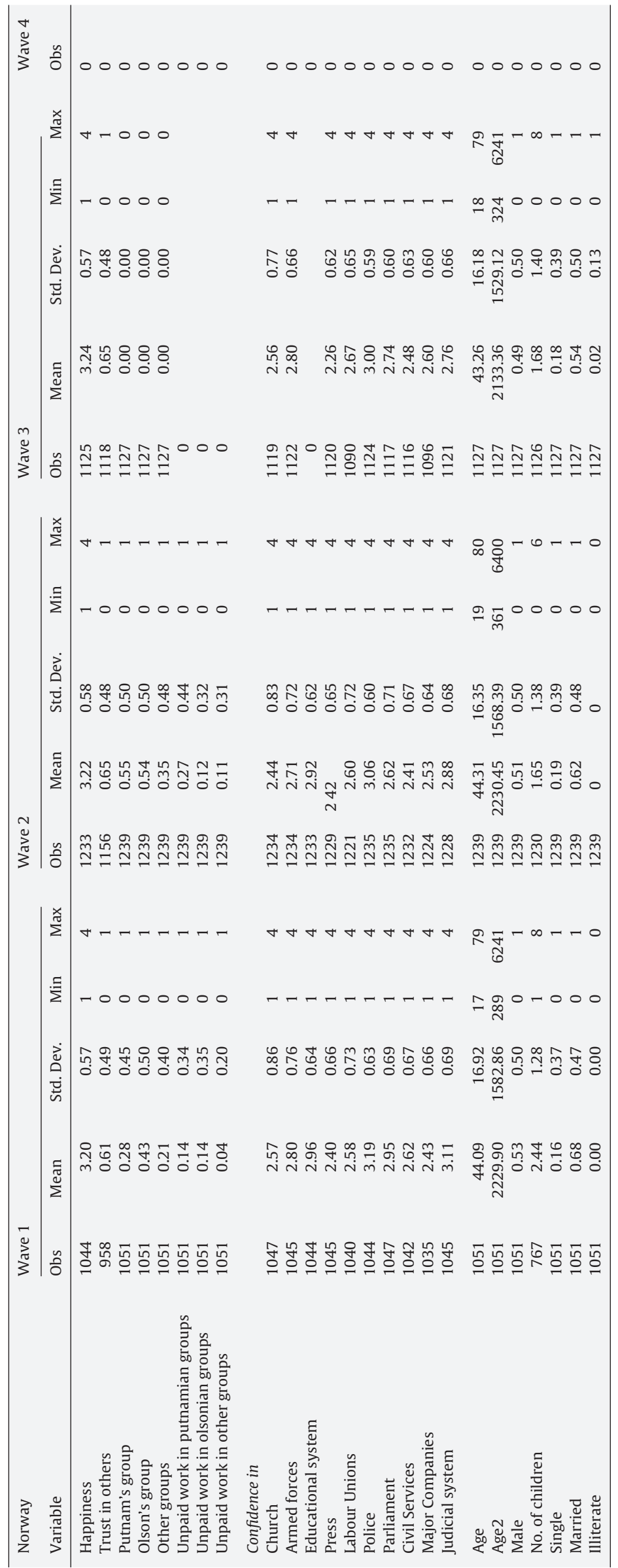




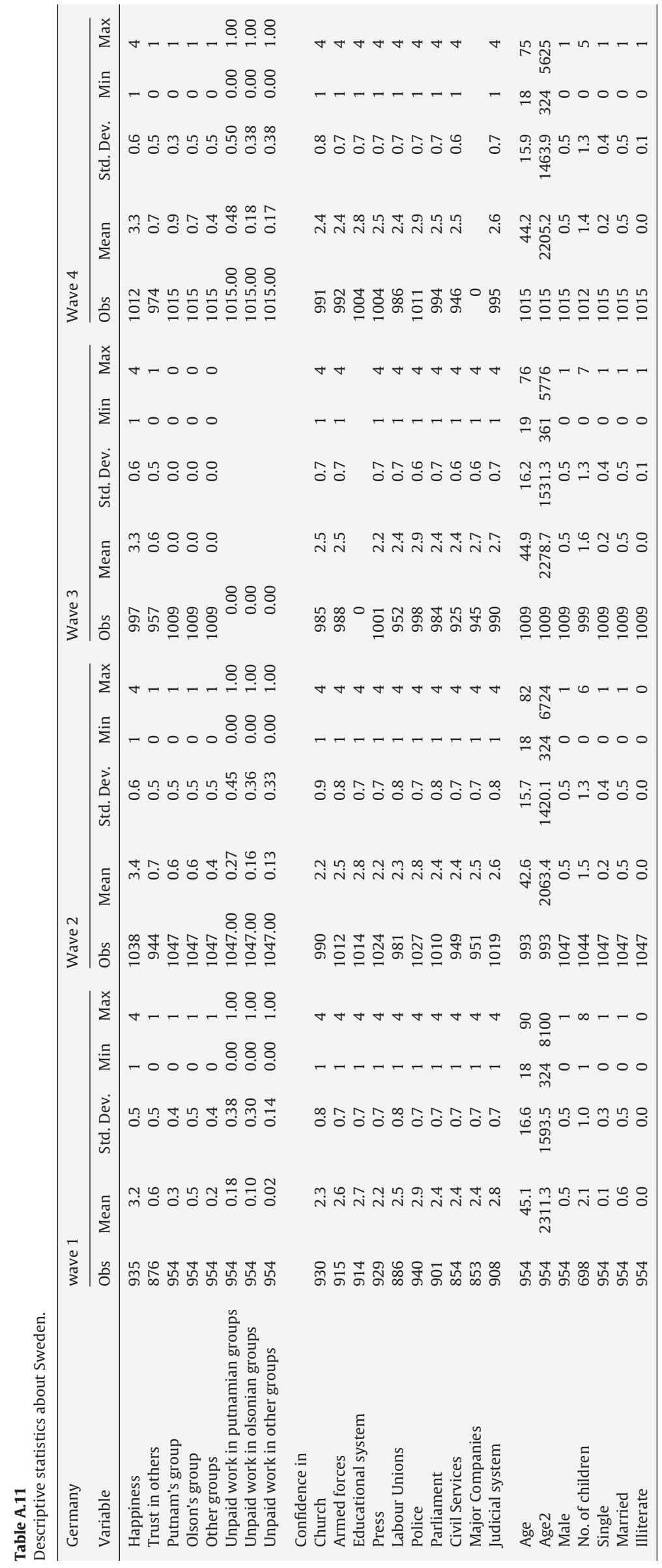




\section{Author's personal copy}

F. Sarracino / The Journal of Socio-Economics 39 (2010) 482-517

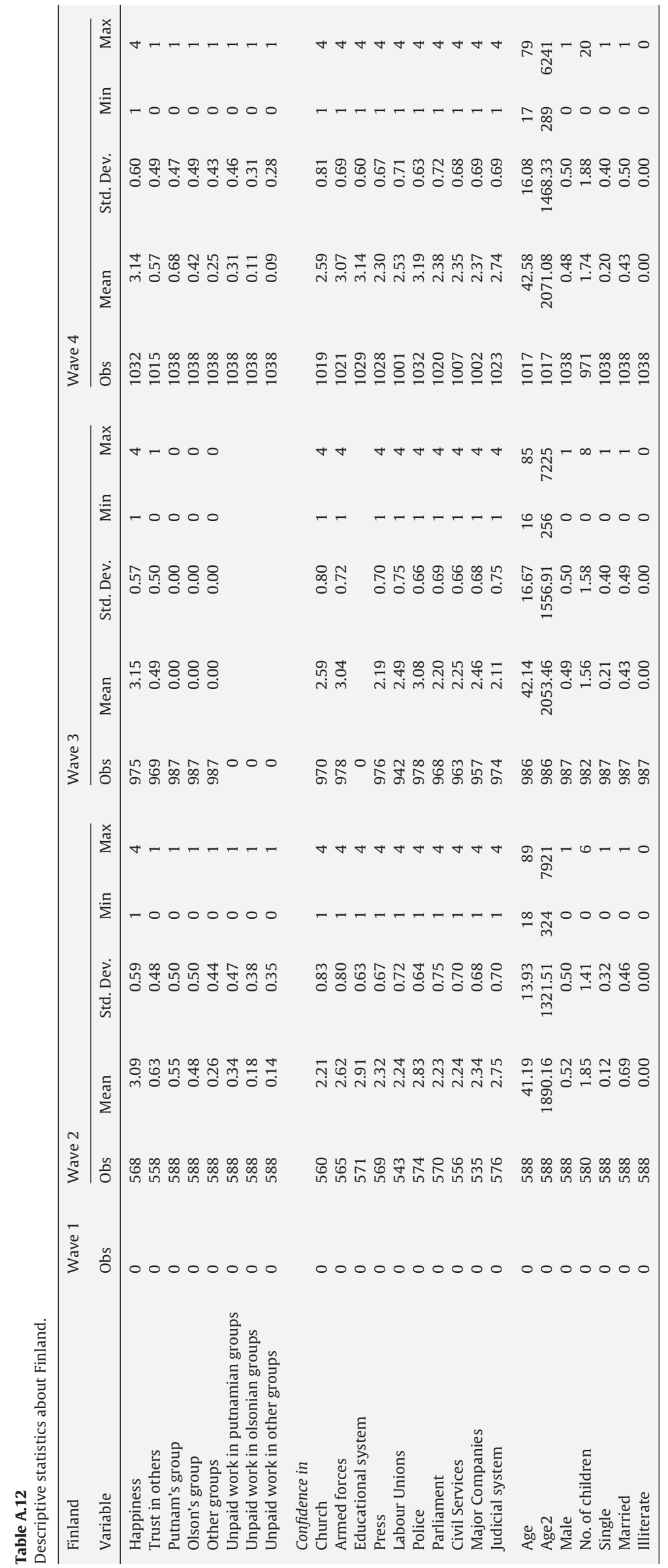


Table A.13

Logit regression about the trends of relational goods and subjective well-being in Italy.

\begin{tabular}{|c|c|c|c|c|c|c|c|c|}
\hline \multirow{2}{*}{$\begin{array}{l}\begin{array}{l}\text { Italy } \\
\text { Putnam's groups }\end{array} \\
\text { W2 }\end{array}$} & \multicolumn{2}{|l|}{ Mean values } & \multicolumn{2}{|c|}{ Demographic controls } & \multicolumn{2}{|l|}{ Familiar status } & \multicolumn{2}{|l|}{ Education } \\
\hline & 0.808 & {$[0.000]^{* * *}$} & 0.841 & {$[0.000]^{* * *}$} & 0.619 & {$[0.000]^{* * *}$} & 0.623 & {$[0.000]^{* * *}$} \\
\hline w4 & 1.145 & {$[0.000]^{* * *}$} & 1.233 & {$[0.000]^{* * *}$} & 1.015 & {$[0.000]^{* * *}$} & 1.035 & {$[0.000]^{* * *}$} \\
\hline Age & & & -0.00892 & {$[0.438]$} & 0.0344 & {$[0.022]^{* *}$} & 0.0320 & {$[0.035]^{* *}$} \\
\hline Age2 & & & -0.0000507 & {$[0.690]$} & -0.000414 & {$[0.008]^{* * *}$} & -0.000381 & {$[0.016]^{* *}$} \\
\hline Male & & & 0.203 & {$[0.002]^{* * *}$} & 0.245 & {$[0.001]^{* * *}$} & 0.241 & {$[0.001]^{* * *}$} \\
\hline No. of children & & & & & -0.0746 & {$[0.053]^{*}$} & -0.0715 & {$[0.065]^{*}$} \\
\hline Single & & & & & 0.482 & {$[0.003]^{* * *}$} & 0.474 & {$[0.003]^{* * *}$} \\
\hline Married & & & & & -0.122 & {$[0.346]$} & -0.131 & {$[0.313]$} \\
\hline Illiterate & & & & & & & -0.343 & [0.169] \\
\hline _cons & -1.929 & {$[0.000]^{* * *}$} & -1.605 & {$[0.000]^{* * *}$} & -2.501 & {$[0.000]^{* * *}$} & -2.462 & {$[0.000]^{* * *}$} \\
\hline No. of observations & 5366 & & 5366 & & 4599 & & 4599 & \\
\hline Pseudo R2 & 0.0278 & & 0.0373 & & 0.0456 & & 0.0460 & \\
\hline chi2 & 145.8 & & 183.6 & & 215.1 & & 218.2 & \\
\hline Olson's groups & Mean values & & Demographic co & atrols & Familiar status & & Education & \\
\hline w2 & 0.198 & {$[0.078]^{*}$} & 0.149 & [0.187] & -0.0145 & [0.912] & -0.0106 & [0.935] \\
\hline w4 & 0.585 & {$[0.000]^{* * *}$} & 0.553 & {$[0.000]^{* * *}$} & 0.393 & {$[0.002]^{* * *}$} & 0.414 & {$[0.002]^{* * *}$} \\
\hline Age & & & 0.150 & {$[0.000]^{* * *}$} & 0.135 & {$[0.000]^{* * *}$} & 0.132 & {$[0.000]^{* * *}$} \\
\hline Age2 & & & -0.00163 & {$[0.000]^{* * *}$} & -0.00146 & {$[0.000]^{* * *}$} & -0.00142 & {$[0.000]^{* * *}$} \\
\hline Male & & & 0.747 & {$[0.000]^{* * *}$} & 0.778 & {$[0.000]^{* * *}$} & 0.772 & {$[0.000]^{* * *}$} \\
\hline No. of children & & & & & -0.143 & {$[0.001]^{* * *}$} & -0.139 & {$[0.001]^{* * *}$} \\
\hline Single & & & & & -0.199 & {$[0.327]$} & -0.207 & {$[0.306]$} \\
\hline Married & & & & & -0.116 & {$[0.447]$} & -0.124 & {$[0.416]$} \\
\hline Illiterate & & & & & & & -0.464 & {$[0.180]$} \\
\hline _cons & -2.163 & {$[0.000]^{* * *}$} & -5.556 & {$[0.000]^{* * *}$} & -4.824 & {$[0.000]^{* * *}$} & -4.772 & {$[0.000]^{* * *}$} \\
\hline No. of observations & 5366 & & 5366 & & 4599 & & 4599 & \\
\hline Pseudo R2 & 0.00842 & & 0.0490 & & 0.0456 & & 0.0461 & \\
\hline chi2 & 35.73 & & 199.6 & & 172.4 & & 174.0 & \\
\hline Other groups & Mean values & & Demographic co & trols & Familiar status & & Education & \\
\hline w2 & 0.275 & {$[0.024]^{* *}$} & 0.292 & {$[0.017]^{* *}$} & 0.153 & {$[0.330]$} & 0.161 & [0.305] \\
\hline w4 & 0.280 & {$[0.022]^{* *}$} & 0.329 & {$[0.008]^{* * *}$} & 0.168 & {$[0.285]$} & 0.206 & {$[0.192]$} \\
\hline Age & & & 0.0162 & {$[0.333]$} & 0.0459 & {$[0.040]^{* *}$} & 0.0412 & {$[0.066]^{*}$} \\
\hline Age2 & & & -0.000280 & [0.130] & -0.000510 & {$[0.028]^{* *}$} & -0.000447 & {$[0.055]^{*}$} \\
\hline Male & & & 0.666 & {$[0.000]^{* * *}$} & 0.597 & {$[0.000]^{* * *}$} & 0.590 & {$[0.000]^{* * *}$} \\
\hline No. of children & & & & & -0.104 & {$[0.038]^{* *}$} & -0.0988 & {$[0.051]^{*}$} \\
\hline Single & & & & & 0.275 & {$[0.251]$} & 0.260 & {$[0.278]$} \\
\hline Married & & & & & 0.0384 & {$[0.837]$} & 0.0248 & {$[0.894]$} \\
\hline Illiterate & & & & & & & -0.977 & {$[0.059]^{*}$} \\
\hline _cons & -2.391 & {$[0.000]^{* * *}$} & -2.896 & {$[0.000]^{* * *}$} & -3.485 & {$[0.000]^{* * *}$} & -3.407 & {$[0.000]^{* * *}$} \\
\hline No. of observations & 5366 & & 5366 & & 4599 & & 4599 & \\
\hline Pseudo R2 & 0.00186 & & 0.0205 & & 0.0217 & & 0.0232 & \\
\hline chi2 & 6.296 & & 86.36 & & 80.05 & & 81.06 & \\
\hline Trust in others & Mean values & & Demographic cor & trols & Familiar status & & Education & \\
\hline w2 & 0.399 & {$[0.000]^{* * *}$} & 0.397 & {$[0.000]^{* * *}$} & 0.411 & {$[0.000]^{* * *}$} & 0.414 & {$[0.000]^{* * *}$} \\
\hline w4 & 0.280 & {$[0.000]^{* * *}$} & 0.306 & {$[0.000]^{* * *}$} & 0.299 & {$[0.004]^{* * *}$} & 0.317 & {$[0.002]^{* * *}$} \\
\hline Age & & & 0.0272 & {$[0.014]^{* *}$} & 0.0662 & {$[0.000]^{* * *}$} & 0.0642 & {$[0.000]^{* * *}$} \\
\hline Age2 & & & -0.000376 & {$[0.002]^{* * *}$} & -0.000709 & {$[0.000]^{* * *}$} & -0.000683 & {$[0.000]^{* * *}$} \\
\hline Male & & & 0.0569 & {$[0.342]$} & 0.0390 & {$[0.552]$} & 0.0360 & {$[0.582]$} \\
\hline No. of children & & & & & -0.0592 & {$[0.076]^{*}$} & -0.0568 & {$[0.090]^{*}$} \\
\hline Single & & & & & 0.300 & {$[0.039]^{* *}$} & 0.293 & {$[0.044]^{* *}$} \\
\hline Married & & & & & -0.00613 & {$[0.958]$} & -0.0132 & [0.909] \\
\hline Illiterate & & & & & & & -0.298 & {$[0.203]$} \\
\hline _cons & -1.005 & {$[0.000]^{* * *}$} & -1.418 & {$[0.000]^{* * *}$} & -2.401 & {$[0.000]^{* * *}$} & -2.368 & {$[0.000]^{* * *}$} \\
\hline No. of observations & 5180 & & 5180 & & 4441 & & 4441 & \\
\hline Pseudo R2 & 0.00407 & & 0.00783 & & 0.0122 & & 0.0125 & \\
\hline chi2 & 25.90 & & 49.19 & & 62.12 & & 62.28 & \\
\hline Unpaid putnamian work & Mean values & & Demographic & controls & Familiar status & & Education & \\
\hline w2 & 0.53 & {$[0.000]^{* * *}$} & 0.533 & {$[0.000]^{* * *}$} & 0.395 & {$[0.009]^{* * *}$} & 0.4 & {$[0.008]^{* * *}$} \\
\hline w4 & 0.77 & {$[0.000]^{* * *}$} & 0.779 & {$[0.000]^{* * *}$} & 0.635 & {$[0.000]^{* * *}$} & 0.661 & {$[0.000]^{* * *}$} \\
\hline Age & & & -0.00266 & {$[0.848]$} & 0.0305 & {$[0.101]$} & 0.0284 & {$[0.129]$} \\
\hline Age2 & & & -0.0001 & [0.519] & -0.000364 & {$[0.064]^{*}$} & -0.000335 & {$[0.091]^{*}$} \\
\hline Male & & & 0.231 & {$[0.003]^{* * *}$} & 0.255 & {$[0.002]^{* * *}$} & 0.251 & {$[0.003]^{* * *}$} \\
\hline No. of children & & & & & -0.0632 & {$[0.148]$} & -0.0602 & {$[0.171]$} \\
\hline Single & & & & & 0.478 & {$[0.014]^{* *}$} & 0.47 & {$[0.016]^{* *}$} \\
\hline Married & & & & & -0.0377 & {$[0.810]$} & -0.045 & {$[0.775]$} \\
\hline Illiterate & & & & & & & -0.445 & {$[0.142]$} \\
\hline _cons & -2.086 & {$[0.000]^{* * *}$} & -1.868 & {$[0.000]^{* * *}$} & -2.651 & {$[0.000]^{* * *}$} & -2.621 & {$[0.000]^{* * *}$} \\
\hline No. of observations & 5366 & & 5366 & & 4599 & & 4599 & \\
\hline Pseudo R2 & 0.0123 & & 0.021 & & 0.0291 & & 0.0297 & \\
\hline chi2 & 46.1 & & 62.11 & & 94.2 & & 98.5 & \\
\hline
\end{tabular}


Table A.13 (Continued)

\begin{tabular}{|c|c|c|c|c|c|c|c|c|}
\hline Happiness & Mean values & & Demograpl & trols & Familiar status & & Education & \\
\hline w2 & 0.356 & {$[0.000]^{* * *}$} & 0.380 & {$[0.000]^{* * *}$} & 0.327 & {$[0.000]^{* * *}$} & 0.330 & {$[0.000]^{* * *}$} \\
\hline w4 & 0.258 & {$[0.001]^{* * *}$} & 0.346 & {$[0.000]^{* * *}$} & 0.290 & {$[0.001]^{* * *}$} & 0.307 & {$[0.001]^{* * *}$} \\
\hline Age & & & 0.0205 & {$[0.042]^{* * *}$} & -0.0506 & {$[0.000]^{* * *}$} & -0.0523 & {$[0.000]^{* * *}$} \\
\hline Age2 & & & -0.000401 & {$[0.000]^{* * *}$} & 0.000316 & {$[0.024]^{* *}$} & 0.000338 & {$[0.017]^{* *}$} \\
\hline Male & & & 0.203 & {$[0.000]^{* * *}$} & 0.174 & {$[0.006]^{* * *}$} & 0.172 & {$[0.007]^{* * *}$} \\
\hline No. of children & & & & & -0.0441 & {$[0.182]$} & -0.0421 & {$[0.201]$} \\
\hline Single & & & & & 0.337 & {$[0.021]^{* *}$} & 0.330 & {$[0.023]^{* *}$} \\
\hline Married & & & & & 1.226 & {$[0.000]^{* * *}$} & 1.220 & {$[0.000]^{* * *}$} \\
\hline Illiterate & & & & & & & -0.238 & {$[0.292]$} \\
\hline cutl & -3.310 & {$[0.000]^{* * *}$} & -3.182 & {$[0.000]^{* * *}$} & -4.118 & {$[0.000]^{* * *}$} & -4.146 & {$[0.000]^{* * *}$} \\
\hline cut2 & -1.298 & {$[0.000]^{* * *}$} & -1.148 & {$[0.000]^{* * *}$} & -2.039 & {$[0.000]^{* * *}$} & -2.067 & {$[0.000]^{* * *}$} \\
\hline cut3 & 1.927 & {$[0.000]^{* * *}$} & 2.133 & {$[0.000]^{* * *}$} & 1.355 & {$[0.000]^{* * *}$} & 1.328 & {$[0.000]^{* * *}$} \\
\hline No. of observations & 5270 & & & & 4519 & & 4519 & \\
\hline Pseudo R2 & 0.00244 & & 0.380 & & 0.0338 & & 0.0340 & \\
\hline chi2 & 26.85 & & & & 271.9 & & 272.2 & \\
\hline
\end{tabular}

$p$-values in brackets: ${ }^{*} p<0.10,{ }^{* *} p<0.05,{ }^{* * *} p<0.01$.

Table A.14

Logit regression about the trends of relational goods and subjective well-being in Great Britain.

\begin{tabular}{|c|c|c|c|c|c|c|c|c|}
\hline \multicolumn{8}{|l|}{ United Kingdom } & Education \\
\hline w2 & 0.224 & {$[0.007]^{* * *}$} & 0.185 & {$[0.029]^{* *}$} & 0.159 & [0.117] & 0.159 & [0.117] \\
\hline w4 & -0.669 & {$[0.000]^{* * *}$} & -0.776 & {$[0.000]^{* * *}$} & -0.786 & {$[0.000]^{* * *}$} & -0.786 & {$[0.000]^{* * *}$} \\
\hline Age & & & 0.0239 & {$[0.035]^{* *}$} & 0.0507 & {$[0.001]^{* * *}$} & 0.0507 & {$[0.001]^{* * *}$} \\
\hline Age2 & & & -0.000201 & {$[0.081]^{*}$} & -0.000443 & {$[0.002]^{* * *}$} & -0.000443 & {$[0.002]^{* * *}$} \\
\hline Male & & & -0.203 & {$[0.006]^{* * *}$} & -0.135 & {$[0.096]^{*}$} & -0.135 & {$[0.096]^{*}$} \\
\hline No. of children & & & & & -0.141 & {$[0.000]^{* * *}$} & -0.141 & {$[0.000]^{* * *}$} \\
\hline Single & & & & & 0.239 & [0.119] & 0.239 & {$[0.119]$} \\
\hline Married & & & & & 0.101 & [0.339] & 0.101 & [0.339] \\
\hline \multicolumn{9}{|l|}{ Illiterate } \\
\hline _cons & -0.736 & {$[0.000]^{* * *}$} & -1.207 & {$[0.000]^{* * *}$} & -1.716 & {$[0.000]^{* * *}$} & -1.716 & {$[0.000]^{* * *}$} \\
\hline No. of observations & 3651 & & 3613 & & 3105 & & 3105 & \\
\hline Pseudo R2 & 0.0208 & & 0.0272 & & 0.0354 & & 0.0354 & \\
\hline chi2 & 87.18 & & 108.1 & & 129.2 & & 129.2 & \\
\hline Olson's groups & \multicolumn{2}{|l|}{ Mean values } & \multicolumn{2}{|c|}{ Demographic controls } & \multicolumn{2}{|l|}{ Familiar status } & \multicolumn{2}{|l|}{ Education } \\
\hline w2 & -0.0873 & {$[0.326]$} & -0.163 & {$[0.075]^{*}$} & -0.0588 & {$[0.590]$} & -0.0588 & {$[0.590]$} \\
\hline w4 & -0.888 & {$[0.000]^{* * *}$} & -1.003 & {$[0.000]^{* * *}$} & -0.864 & {$[0.000]^{* * *}$} & -0.864 & {$[0.000]^{* * *}$} \\
\hline Age & & & 0.121 & {$[0.000]^{* * *}$} & 0.159 & {$[0.000]^{* * *}$} & 0.159 & {$[0.000]^{* * *}$} \\
\hline Age2 & & & -0.00134 & {$[0.000]^{* * *}$} & -0.00166 & {$[0.000]^{* * *}$} & -0.00166 & {$[0.000]^{* * *}$} \\
\hline Male & & & 0.749 & {$[0.000]^{* * *}$} & 0.839 & {$[0.000]^{* * *}$} & 0.839 & {$[0.000]^{* * *}$} \\
\hline No. of children & & & & & -0.132 & {$[0.000]^{* * *}$} & -0.132 & {$[0.000]^{* * *}$} \\
\hline Single & & & & & 0.224 & {$[0.251]$} & 0.224 & {$[0.251]$} \\
\hline Married & & & & & 0.164 & {$[0.200]$} & 0.164 & {$[0.200]$} \\
\hline \multicolumn{9}{|l|}{ Illiterate } \\
\hline _cons & -0.986 & {$[0.000]^{* * *}$} & -3.593 & {$[0.000]^{* * *}$} & -4.663 & {$[0.000]^{* * *}$} & -4.663 & {$[0.000]^{* * *}$} \\
\hline No. of observations & 3651 & & 3613 & & 3105 & & 3105 & \\
\hline Pseudo R2 & 0.0194 & & 0.0658 & & 0.0802 & & 0.0802 & \\
\hline chi2 & 67.43 & & 186.1 & & 196.2 & & 196.2 & \\
\hline Other groups & \multicolumn{2}{|l|}{ Mean values } & \multicolumn{2}{|c|}{ Demographic controls } & \multicolumn{2}{|l|}{ Familiar status } & \multicolumn{2}{|l|}{ Education } \\
\hline w2 & 0.504 & {$[0.000]^{* * *}$} & 0.460 & {$[0.000]^{* * *}$} & 0.589 & {$[0.000]^{* * *}$} & 0.589 & {$[0.000]^{* * *}$} \\
\hline w4 & 0.0613 & {$[0.644]$} & -0.0314 & {$[0.817]$} & 0.145 & {$[0.375]$} & 0.145 & {$[0.375]$} \\
\hline Age & & & 0.0595 & {$[0.000]^{* * *}$} & 0.0811 & {$[0.000]^{* * *}$} & 0.0811 & {$[0.000]^{* * *}$} \\
\hline Age2 & & & -0.000636 & {$[0.000]^{* * *}$} & -0.000816 & {$[0.000]^{* * *}$} & -0.000816 & {$[0.000]^{* * *}$} \\
\hline Male & & & -0.00826 & {$[0.931]$} & -0.111 & {$[0.290]$} & -0.111 & {$[0.290]$} \\
\hline No. of children & & & & & -0.0712 & {$[0.060]^{*}$} & -0.0712 & {$[0.060]^{*}$} \\
\hline Single & & & & & -0.0736 & [0.717] & -0.0736 & {$[0.717]$} \\
\hline Married & & & & & 0.227 & {$[0.104]$} & 0.227 & {$[0.104]$} \\
\hline \multicolumn{9}{|l|}{ Illiterate } \\
\hline _cons & -2.026 & {$[0.000]^{* * *}$} & -3.163 & {$[0.000]^{* * *}$} & -3.821 & {$[0.000]^{* * *}$} & -3.821 & {$[0.000]^{* * *}$} \\
\hline No. of observations & 3651 & & 3613 & & 3105 & & 3105 & \\
\hline Pseudo R2 & 0.00843 & & 0.0149 & & 0.0220 & & 0.0220 & \\
\hline chi2 & 25.39 & & 41.24 & & 53.22 & & 53.22 & \\
\hline Trust in others & Mean values & & Demographic cont & rols & Familiar status & & Education & \\
\hline w2 & 0.0227 & [0.777] & -0.0367 & [0.655] & -0.0405 & [0.678] & -0.0434 & [0.657] \\
\hline w3 & -0.588 & {$[0.000]^{* * *}$} & -0.642 & {$[0.000]^{* * *}$} & -0.743 & {$[0.000]^{* * *}$} & -0.596 & {$[0.000]^{* * *}$} \\
\hline W4 & -0.626 & {$[0.000]^{* * *}$} & -0.660 & {$[0.000]^{* * *}$} & -0.646 & {$[0.000]^{* * *}$} & -0.644 & {$[0.000]^{* * *}$} \\
\hline Age & & & 0.0442 & {$[0.000]^{* * *}$} & 0.0610 & {$[0.000]^{* * *}$} & 0.0617 & {$[0.000]^{* * *}$} \\
\hline Age2 & & & -0.000409 & {$[0.000]^{* * *}$} & -0.000556 & {$[0.000]^{* * *}$} & -0.000549 & {$[0.000]^{* * *}$} \\
\hline Male & & & 0.265 & {$[0.000]^{* * *}$} & 0.275 & {$[0.000]^{* * *}$} & 0.267 & {$[0.000]^{* * *}$} \\
\hline
\end{tabular}


Table A.14 (Continued)

\begin{tabular}{|c|c|c|c|c|c|c|c|c|}
\hline \multirow{2}{*}{$\begin{array}{l}\text { Trust in others } \\
\text { No. of children }\end{array}$} & \multicolumn{2}{|l|}{ Mean values } & \multicolumn{2}{|c|}{ Demographic controls } & \multicolumn{2}{|l|}{ Familiar status } & \multicolumn{2}{|l|}{ Education } \\
\hline & & & & & -0.0820 & {$[0.002]^{* * *}$} & -0.0895 & {$[0.001]^{* * *}$} \\
\hline Single & & & & & 0.165 & {$[0.191]$} & 0.155 & {$[0.219]$} \\
\hline Married & & & & & 0.0949 & [0.279] & 0.0920 & {$[0.294]$} \\
\hline Illiterate & & & & & & & -0.538 & {$[0.001]^{* * *}$} \\
\hline _cons & -0.277 & {$[0.000]^{* * *}$} & -1.376 & {$[0.000]^{* * *}$} & -1.729 & {$[0.000]^{* * *}$} & -1.755 & {$[0.000]^{* * *}$} \\
\hline No. of observations & 4600 & & 4563 & & 4076 & & 4076 & \\
\hline Pseudo R2 & 0.0163 & & 0.0233 & & 0.0281 & & 0.0304 & \\
\hline chi2 & 96.70 & & 136.8 & & 147.3 & & 156.3 & \\
\hline Unpaid putnamian work & Mean values & & Demographi & ntrols & Familiar status & & Education & \\
\hline w2 & 0.295 & {$[0.015]^{* *}$} & 0.286 & {$[0.019]^{* *}$} & 0.254 & {$[0.068]^{*}$} & 0.254 & {$[0.068]^{*}$} \\
\hline w4 & 1.06 & {$[0.000]^{* * *}$} & 1.063 & {$[0.000]^{* * *}$} & 1.083 & {$[0.000]^{* * *}$} & 1.083 & {$[0.000]^{* * *}$} \\
\hline Age & & & 0.0823 & {$[0.000]^{* * *}$} & 0.0966 & {$[0.000]^{* * *}$} & 0.0966 & {$[0.000]^{* * *}$} \\
\hline Age2 & & & -0.00075 & {$[0.000]^{* * *}$} & -0.000872 & {$[0.000]^{* * *}$} & -0.000872 & {$[0.000]^{* * *}$} \\
\hline Male & & & -0.255 & {$[0.008]^{* * *}$} & -0.228 & {$[0.025]^{* *}$} & -0.228 & {$[0.025]^{* *}$} \\
\hline No. of children & & & & & -0.12 & {$[0.002]^{* * *}$} & -0.12 & {$[0.002]^{* * *}$} \\
\hline Single & & & & & 0.179 & {$[0.345]$} & 0.179 & {$[0.345]$} \\
\hline Married & & & & & 0.243 & {$[0.079]^{*}$} & 0.243 & {$[0.079]^{*}$} \\
\hline Illiterate & & & & & & & & \\
\hline _cons & -1.844 & {$[0.000]^{* * *}$} & -3.678 & {$[0.000]^{* * *}$} & -4.009 & {$[0.000]^{* * *}$} & -4.009 & {$[0.000]^{* * *}$} \\
\hline No. of observations & 3651 & & 3613 & & 3105 & & 3105 & \\
\hline Pseudo R2 & 0.03 & & 0.0448 & & 0.0442 & & 0.0442 & \\
\hline chi2 & 90.62 & & 134.1 & & 116.5 & & 116.5 & \\
\hline Happiness & Mean values & & Demographic & trols & Familiar status & & Education & \\
\hline w2 & -0.206 & {$[0.006]^{* * *}$} & -0.232 & {$[0.002]^{* * *}$} & -0.182 & {$[0.048]^{* *}$} & -0.183 & {$[0.048]^{* *}$} \\
\hline w3 & -0.297 & {$[0.000]^{* * *}$} & -0.320 & {$[0.000]^{* * *}$} & -0.313 & {$[0.003]^{* * *}$} & -0.272 & {$[0.015]^{* *}$} \\
\hline Age & & & 0.0149 & {$[0.111]$} & -0.0237 & {$[0.052]^{*}$} & -0.0236 & {$[0.054]^{*}$} \\
\hline Age2 & & & -0.000132 & {$[0.167]$} & 0.000258 & {$[0.030]^{* *}$} & 0.000261 & {$[0.028]^{* *}$} \\
\hline Male & & & -0.0612 & {$[0.348]$} & -0.0874 & {$[0.225]$} & -0.0896 & {$[0.213]$} \\
\hline No. of children & & & & & -0.0782 & {$[0.008]^{* * *}$} & -0.0810 & {$[0.006]^{* * *}$} \\
\hline Single & & & & & 0.0349 & {$[0.806]$} & 0.0286 & {$[0.841]$} \\
\hline Married & & & & & 0.875 & {$[0.000]^{* * *}$} & 0.875 & {$[0.000]^{* * *}$} \\
\hline Illiterate & & & & & & & -0.146 & {$[0.304]$} \\
\hline cut1 & -4.414 & {$[0.000]^{* * *}$} & -4.123 & {$[0.000]^{* * *}$} & -4.470 & {$[0.000]^{* * *}$} & -4.463 & {$[0.000]^{* * *}$} \\
\hline cut2 & -2.649 & {$[0.000]^{* * *}$} & -2.342 & {$[0.000]^{* * *}$} & -2.686 & {$[0.000]^{* * *}$} & -2.678 & {$[0.000]^{* * *}$} \\
\hline cut3 & 0.436 & {$[0.000]^{* * *}$} & 0.747 & {$[0.000]^{* * *}$} & 0.375 & {$[0.204]$} & 0.383 & {$[0.195]$} \\
\hline No. of observations & 3728 & & & & 3224 & & 3224 & \\
\hline Pseudo R2 & 0.00201 & & -0.232 & & 0.0214 & & 0.0215 & \\
\hline chi2 & 14.58 & & & & 121.8 & & 121.7 & \\
\hline
\end{tabular}

p-values in brackets: ${ }^{*} p<0.10,{ }^{* *} p<0.05,{ }^{* * *} p<0.01$.

Table A.15

Logit regression about the trends of relational goods and subjective well-being in Ireland.

\begin{tabular}{|c|c|c|c|c|c|c|c|c|}
\hline \multirow{2}{*}{$\begin{array}{l}\text { Ireland } \\
\text { Putnam's groups } \\
\text { W2 }\end{array}$} & \multicolumn{2}{|l|}{ Mean values } & \multicolumn{2}{|c|}{ Demographic controls } & \multicolumn{2}{|l|}{ Familiar status } & \multicolumn{2}{|l|}{ Education } \\
\hline & 0.0399 & {$[0.648]$} & 0.0174 & {$[0.845]$} & -0.0846 & {$[0.451]$} & -0.0790 & {$[0.481]$} \\
\hline W4 & 0.239 & {$[0.006]^{* * *}$} & 0.218 & {$[0.015]^{* *}$} & 0.162 & [0.148] & 0.256 & {$[0.030]^{* *}$} \\
\hline Age & & & 0.0167 & {$[0.141]$} & 0.0222 & [0.165] & 0.0208 & {$[0.192]$} \\
\hline Age2 & & & -0.000200 & {$[0.086]^{*}$} & -0.000232 & {$[0.141]$} & -0.000202 & {$[0.200]$} \\
\hline Male & & & 0.220 & {$[0.002]^{* * *}$} & 0.233 & {$[0.005]^{* * *}$} & 0.246 & {$[0.003]^{* * *}$} \\
\hline No. of children & & & & & 0.00607 & {$[0.803]$} & 0.0113 & {$[0.641]$} \\
\hline Single & & & & & 0.835 & {$[0.000]^{* * *}$} & 0.837 & {$[0.000]^{* * *}$} \\
\hline Married & & & & & 0.398 & {$[0.005]^{* * * *}$} & 0.395 & {$[0.005]^{* * *}$} \\
\hline Illiterate & & & & & & & -0.413 & {$[0.014]^{* *}$} \\
\hline cons & -0.449 & {$[0.000]^{* * *}$} & -0.820 & {$[0.001]^{* * *}$} & -1.409 & {$[0.000]^{* * *}$} & -1.438 & {$[0.000]^{* * *}$} \\
\hline No. of observations & 3229 & & 3200 & & 2572 & & 2572 & \\
\hline Pseudo R2 & 0.00194 & & 0.00497 & & 0.0151 & & 0.0168 & \\
\hline chi2 & 8.516 & & 21.04 & & 49.66 & & 56.53 & \\
\hline Olson's groups & Mean values & & Demographic & trols & Familiar status & & Education & \\
\hline w2 & 0.0467 & [0.683] & 0.0105 & [0.928] & 0.207 & [0.175] & 0.211 & [0.167] \\
\hline w4 & 0.328 & {$[0.003]^{* * *}$} & 0.322 & {$[0.004]^{* * *}$} & 0.501 & {$[0.001]^{* * * *}$} & 0.593 & {$[0.000]^{* * *}$} \\
\hline Age & & & 0.0610 & {$[0.000]^{* * *}$} & 0.108 & {$[0.000]^{* * *}$} & 0.107 & {$[0.000]^{* * *}$} \\
\hline Age2 & & & -0.000824 & {$[0.000]^{* * *}$} & -0.00124 & {$[0.000]^{* * *}$} & -0.00121 & {$[0.000]^{* * *}$} \\
\hline Male & & & 0.712 & {$[0.000]^{* * *}$} & 0.694 & {$[0.000]^{* * *}$} & 0.712 & {$[0.000]^{* * *}$} \\
\hline No. of children & & & & & -0.0220 & {$[0.486]$} & -0.0152 & {$[0.631]$} \\
\hline Single & & & & & 0.281 & {$[0.257]$} & 0.289 & {$[0.245]$} \\
\hline Married & & & & & 0.180 & {$[0.386]$} & 0.173 & {$[0.404]$} \\
\hline Illiterate & & & & & & & -0.499 & {$[0.029]^{* *}$} \\
\hline _cons & -1.632 & {$[0.000]^{* * *}$} & -2.853 & {$[0.000]^{* * *}$} & -4.317 & {$[0.000]^{* * *}$} & -4.367 & {$[0.000]^{* * *}$} \\
\hline No. of observations & 3229 & & 3200 & & 2572 & & 2572 & \\
\hline Pseudo R2 & 0.00337 & & 0.0397 & & 0.0482 & & 0.0503 & \\
\hline chi2 & 10.45 & & 115.7 & & 115.6 & & 122.0 & \\
\hline
\end{tabular}


Table A.15 (Continued)

\begin{tabular}{|c|c|c|c|c|c|c|c|c|}
\hline Other groups & Mean values & & Demographic con & trols & Familiar status & & Education & \\
\hline w2 & 0.266 & {$[0.029]^{* *}$} & 0.313 & {$[0.010]^{* *}$} & 0.463 & {$[0.005]^{* * *}$} & 0.466 & {$[0.004]^{* * *}$} \\
\hline w4 & 0.555 & {$[0.000]^{* * *}$} & 0.609 & {$[0.000]^{* * *}$} & 0.733 & {$[0.000]^{* * *}$} & 0.777 & {$[0.000]^{* * *}$} \\
\hline Age & & & -0.0126 & {$[0.437]$} & 0.0300 & [0.177] & 0.0293 & [0.189] \\
\hline Age2 & & & 0.0000220 & {$[0.896]$} & -0.000387 & {$[0.081]^{*}$} & -0.000370 & {$[0.097]^{*}$} \\
\hline Male & & & 0.139 & [0.157] & 0.0716 & [0.523] & 0.0790 & {$[0.480]$} \\
\hline No. of children & & & & & 0.0309 & [0.322] & 0.0338 & {$[0.280]$} \\
\hline Single & & & & & 0.174 & {$[0.455]$} & 0.175 & {$[0.452]$} \\
\hline Married & & & & & 0.0439 & {$[0.815]$} & 0.0410 & [0.827] \\
\hline Illiterate & & & & & & & -0.209 & [0.323] \\
\hline _cons & -1.932 & {$[0.000]^{* * *}$} & -1.547 & {$[0.000]^{* * *}$} & -2.774 & {$[0.000]^{* * *}$} & -2.790 & {$[0.000]^{* * *}$} \\
\hline No. of observations & 3229 & & 3200 & & 2572 & & 2572 & \\
\hline Pseudo R2 & 0.00810 & & 0.0133 & & 0.0150 & & 0.0155 & \\
\hline chi2 & 22.86 & & 42.57 & & 37.74 & & 38.90 & \\
\hline Trust in others & Mean values & & Demographic con & rols & Familiar status & & Education & \\
\hline w2 & 0.254 & {$[0.004]^{* * *}$} & 0.218 & {$[0.014]^{* *}$} & 0.131 & {$[0.240]$} & 0.133 & {$[0.230]$} \\
\hline w4 & 0.217 & {$[0.015]^{* *}$} & -0.235 & {$[0.010]^{* * *}$} & -0.289 & {$[0.010]^{* *}$} & -0.244 & {$[0.041]^{* *}$} \\
\hline Age & & & 0.0213 & {$[0.061]^{*}$} & 0.0376 & {$[0.020]^{* *}$} & 0.0369 & {$[0.022]^{* *}$} \\
\hline Age2 & & & -0.000202 & {$[0.084]^{*}$} & -0.000275 & {$[0.082]^{*}$} & -0.000261 & {$[0.102]$} \\
\hline Male & & & 0.233 & {$[0.001]^{* * *}$} & 0.140 & {$[0.096]^{*}$} & 0.145 & {$[0.083]^{*}$} \\
\hline No. of children & & & & & -0.00697 & {$[0.776]$} & -0.00456 & {$[0.853]$} \\
\hline Single & & & & & 0.791 & {$[0.000]^{* * *}$} & 0.794 & {$[0.000]^{* * *}$} \\
\hline Married & & & & & 0.598 & {$[0.000]^{* * *}$} & 0.598 & {$[0.000]^{* * *}$} \\
\hline Illiterate & & & & & & & -0.192 & {$[0.260]$} \\
\hline _cons & -0.359 & {$[0.000]^{* * *}$} & -0.922 & {$[0.000]^{* * *}$} & -1.938 & {$[0.000]^{* * *}$} & -1.952 & {$[0.000]^{* * *}$} \\
\hline No. of observations & 3150 & & 3121 & & 2520 & & 2520 & \\
\hline Pseudo R2 & 0.00621 & & 0.00891 & & 0.0176 & & 0.0180 & \\
\hline Chi2 & 26.37 & & 37.92 & & 58.75 & & 59.35 & \\
\hline Unpaid putnamian work & Mean values & & Demographic & controls & Familiar status & & Education & \\
\hline w2 & 0.383 & {$[0.001]^{* * *}$} & 0.345 & {$[0.003]^{* * *}$} & 0.326 & {$[0.026]^{* *}$} & 0.343 & {$[0.019]^{* *}$} \\
\hline w4 & 0.538 & {$[0.000]^{* * *}$} & 0.491 & {$[0.000]^{* * *}$} & 0.516 & {$[0.000]^{* * *}$} & 0.727 & {$[0.000]^{* * *}$} \\
\hline Age & & & 0.0572 & {$[0.000]^{* * *}$} & 0.0414 & {$[0.047]^{* *}$} & 0.0431 & {$[0.040]^{* *}$} \\
\hline Age2 & & & -0.000616 & {$[0.000]^{* * *}$} & -0.00044 & {$[0.034]^{* *}$} & -0.000423 & {$[0.043]^{* *}$} \\
\hline Male & & & 0.0964 & {$[0.300]$} & 0.0772 & {$[0.458]$} & 0.0929 & {$[0.374]$} \\
\hline No. of children & & & & & 0.0275 & [0.362] & 0.0403 & [0.187] \\
\hline Single & & & & & 0.818 & {$[0.000]^{* * *}$} & 0.827 & {$[0.000]^{* * *}$} \\
\hline Married & & & & & 0.731 & {$[0.000]^{* * *}$} & 0.725 & {$[0.000]^{* * *}$} \\
\hline Illiterate & & & & & & & -0.797 & {$[0.000]^{* * *}$} \\
\hline _cons & -1.733 & {$[0.000]^{* * *}$} & 2.888 & {$[0.000]^{* * *}$} & -3.327 & {$[0.000]^{* * *}$} & -3.494 & {$[0.000]^{* * *}$} \\
\hline No. of observations & 3229 & & 3200 & & 2572 & & 2572 & \\
\hline Pseudo R2 & 0.00816 & & 0.0136 & & 0.0172 & & 0.0235 & \\
\hline chi2 & 23.04 & & 41.78 & & 38.01 & & 56.79 & \\
\hline Happiness & Mean values & & Demographic cor & trols & Familiar status & & Education & \\
\hline w2 & 0.0534 & [0.539] & 0.0818 & [0.355] & -0.0359 & {$[0.744]$} & -0.0376 & {$[0.733]$} \\
\hline w4 & 0.0687 & {$[0.405]$} & 0.0823 & {$[0.335]$} & 0.0359 & {$[0.737]$} & 0.0123 & [0.913] \\
\hline Age & & & 0.00607 & [0.584] & -0.0590 & {$[0.000]^{* * *}$} & -0.0587 & {$[0.000]^{* * * *}$} \\
\hline Age2 & & & -0.000121 & {$[0.290]$} & 0.000551 & {$[0.000]^{* * *}$} & 0.000544 & {$[0.000]^{* * *}$} \\
\hline Male & & & -0.311 & {$[0.000]^{* * *}$} & -0.342 & {$[0.000]^{* * *}$} & -0.345 & {$[0.000]^{* * *}$} \\
\hline No. of children & & & & & -0.0372 & {$[0.126]$} & -0.0386 & [0.116] \\
\hline Single & & & & & 0.217 & {$[0.226]$} & 0.219 & [0.224] \\
\hline Married & & & & & 1.019 & {$[0.000]^{* * *}$} & 1.021 & {$[0.000]^{* * *}$} \\
\hline Illiterate & & & & & & & 0.102 & [0.526] \\
\hline cut1 & -4.795 & {$[0.000]^{* * *}$} & -4.939 & {$[0.000]^{* * *}$} & -5.892 & {$[0.000]^{* * *}$} & -5.898 & {$[0.000]^{* * *}$} \\
\hline cut2 & -2.976 & {$[0.000]^{* * *}$} & -3.134 & {$[0.000]^{* * *}$} & -3.997 & {$[0.000]^{* * *}$} & -4.004 & {$[0.000]^{* * *}$} \\
\hline cut3 & 0.355 & {$[0.000]^{* * *}$} & 0.229 & {$[0.331]$} & -0.648 & {$[0.081]^{*}$} & -0.654 & {$[0.079]^{*}$} \\
\hline No. of observations & 3163 & & & & 2531 & & 2531 & \\
\hline Pseudo R2 & 0.000137 & & 0.0818 & & 0.0234 & & 0.0235 & \\
\hline chi2 & 0.770 & & & & 94.58 & & 94.60 & \\
\hline
\end{tabular}

-values in brackets: ${ }^{*} p<0.10,{ }^{* *} p<0.05,{ }^{* * *} p<0.01$. 
Table A.16

Logit regression about the trends of relational goods and subjective well-being in France.

\begin{tabular}{|c|c|c|c|c|c|c|c|c|}
\hline $\begin{array}{l}\text { France } \\
\text { Putnam's groups }\end{array}$ & Mean values & & Demographic con & trols & Familiar status & & Education & \\
\hline w2 & 1.045 & {$[0.000]^{* * *}$} & 1.031 & {$[0.000]^{* * *}$} & 0.930 & {$[0.000]^{* * *}$} & 0.949 & {$[0.000]^{* * *}$} \\
\hline w4 & 0.990 & {$[0.000]^{* * *}$} & 0.966 & {$[0.000]^{* * *}$} & 0.862 & {$[0.000]^{* * *}$} & 0.998 & {$[0.000]^{* * *}$} \\
\hline Age & & & 0.0450 & {$[0.000]^{* * *}$} & 0.0400 & {$[0.007]^{* * *}$} & 0.0373 & {$[0.012]^{* *}$} \\
\hline Age2 & & & -0.000505 & {$[0.000]^{* * *}$} & -0.000458 & {$[0.002]^{* * *}$} & -0.000394 & {$[0.008]^{* * *}$} \\
\hline Male & & & 0.133 & {$[0.082]^{*}$} & 0.145 & {$[0.074]^{*}$} & 0.143 & {$[0.079]^{*}$} \\
\hline No. of children & & & & & -0.0205 & [0.509] & -0.00766 & {$[0.807]$} \\
\hline Single & & & & & 0.153 & {$[0.277]$} & 0.146 & {$[0.301]$} \\
\hline Married & & & & & 0.0848 & {$[0.431]$} & 0.0900 & {$[0.405]$} \\
\hline Illiterate & & & & & & & -0.793 & {$[0.000]^{* * *}$} \\
\hline _cons & -1.872 & {$[0.000]^{* * *}$} & -2.775 & {$[0.000]^{* * *}$} & -2.614 & {$[0.000]^{* * *}$} & -2.670 & {$[0.000]^{* * *}$} \\
\hline No. of observations & 3817 & & 3817 & & 3383 & & 3383 & \\
\hline Pseudo R2 & 0.0305 & & 0.0347 & & 0.0249 & & 0.0308 & \\
\hline chi2 & 113.6 & & 132.9 & & 86.27 & & 107.7 & \\
\hline Olson's groups & Mean values & & Demographic co & ntrols & Familiar status & & Education & \\
\hline w2 & 0.0442 & [0.735] & -0.0706 & [0.596] & -0.0941 & {$[0.518]$} & -0.0878 & {$[0.547]$} \\
\hline w4 & -0.141 & {$[0.240]$} & -0.337 & {$[0.006]^{* * *}$} & -0.354 & {$[0.012]^{* *}$} & -0.279 & {$[0.059]^{*}$} \\
\hline Age & & & 0.198 & {$[0.000]^{* * *}$} & 0.192 & {$[0.000]^{* * *}$} & 0.191 & {$[0.000]^{* * *}$} \\
\hline Age2 & & & -0.00202 & {$[0.000]^{* * *}$} & -0.00195 & {$[0.000]^{* * *}$} & -0.00192 & {$[0.000]^{* * *}$} \\
\hline Male & & & 0.788 & {$[0.000]^{* * *}$} & 0.753 & {$[0.000]^{* * *}$} & 0.753 & {$[0.000]^{* * *}$} \\
\hline No. of children & & & & & -0.0921 & {$[0.026]^{* *}$} & -0.0880 & {$[0.034]^{* *}$} \\
\hline Single & & & & & -0.0325 & {$[0.887]$} & -0.0418 & {$[0.854]$} \\
\hline Married & & & & & 0.355 & {$[0.026]^{* *}$} & 0.362 & {$[0.024]^{* *}$} \\
\hline Illiterate & & & & & & & -0.409 & {$[0.098]^{*}$} \\
\hline _cons & -1.992 & {$[0.000]^{* * *}$} & -6.586 & {$[0.000]^{* * *}$} & -6.526 & {$[0.000]^{* * *}$} & -6.549 & {$[0.000]^{* * *}$} \\
\hline No. of observations & 3817 & & 3817 & & 3383 & & 3383 & \\
\hline Pseudo R2 & 0.000936 & & 0.0578 & & 0.0606 & & 0.0618 & \\
\hline chi2 & 2.537 & & 141.5 & & 141.7 & & 141.4 & \\
\hline Other groups & Mean values & & Demographic cor & trols & Familiar status & & Education & \\
\hline w2 & 0.340 & {$[0.021]^{* *}$} & 0.313 & {$[0.033]^{* *}$} & 0.571 & {$[0.001]^{* * *}$} & 0.588 & {$[0.001]^{* * *}$} \\
\hline w4 & 0.371 & {$[0.005]^{* * *}$} & 0.322 & {$[0.016]^{* *}$} & 0.573 & {$[0.001]^{* * *}$} & 0.708 & {$[0.000]^{* * *}$} \\
\hline Age & & & 0.0589 & {$[0.002]^{* * *}$} & 0.0707 & {$[0.002]^{* * *}$} & 0.0679 & {$[0.003]^{* * *}$} \\
\hline Age2 & & & -0.000635 & {$[0.001]^{* * *}$} & -0.000714 & {$[0.002]^{* * *}$} & -0.000652 & {$[0.004]^{* * *}$} \\
\hline Male & & & 0.345 & {$[0.002]^{* * *}$} & 0.258 & {$[0.033]^{* *}$} & 0.256 & {$[0.035]^{* *}$} \\
\hline No. of children & & & & & 0.0301 & {$[0.491]$} & 0.0419 & {$[0.343]$} \\
\hline Single & & & & & 0.419 & {$[0.059]^{*}$} & 0.410 & {$[0.065]^{*}$} \\
\hline Married & & & & & 0.418 & {$[0.014]^{* *}$} & 0.424 & {$[0.012]^{* *}$} \\
\hline Illiterate & & & & & & & -0.800 & {$[0.003]^{* * *}$} \\
\hline _cons & -2.454 & {$[0.000]^{* * *}$} & -3.785 & {$[0.000]^{* * *}$} & -4.737 & {$[0.000]^{* * *}$} & -4.793 & {$[0.000]^{* * *}$} \\
\hline No. of observations & 3817 & & 3817 & & 3383 & & 3383 & \\
\hline Pseudo R2 & 0.00358 & & 0.0117 & & 0.0183 & & 0.0232 & \\
\hline chi2 & 8.487 & & 30.29 & & 40.38 & & 49.42 & \\
\hline Trust in others & Mean values & & Demographic con & trols & Familiar status & & Education & \\
\hline w2 & -0.111 & {$[0.288]$} & -0.111 & {$[0.288]$} & -0.0994 & {$[0.420]$} & -0.0737 & [0.549] \\
\hline w4 & -0.195 & {$[0.036]^{* *}$} & -0.190 & {$[0.044]^{* *}$} & -0.228 & {$[0.048]^{* *}$} & -0.0588 & {$[0.622]$} \\
\hline Age & & & 0.0209 & [0.127] & 0.0569 & {$[0.001]^{* * *}$} & 0.0538 & {$[0.002]^{* * *}$} \\
\hline Age2 & & & -0.000280 & {$[0.055]^{*}$} & $-0.00057]$ & {$[0.002]^{* * *}$} & -0.000499 & {$[0.005]^{* * *}$} \\
\hline Male & & & 0.0665 & {$[0.403]$} & -0.0282 & {$[0.749]$} & -0.0341 & {$[0.699]$} \\
\hline No. of children & & & & & -0.0728 & {$[0.039]^{* *}$} & -0.0599 & {$[0.094]^{*}$} \\
\hline Single & & & & & 0.444 & {$[0.004]^{* * *}$} & 0.429 & {$[0.005]^{* * *}$} \\
\hline Married & & & & & 0.00317 & {$[0.979]$} & 0.0112 & {$[0.926]$} \\
\hline Illiterate & & & & & & & -1.083 & {$[0.000]^{* * *}$} \\
\hline _cons & -1.109 & {$[0.000]^{* * *}$} & -1.443 & {$[0.000]^{* * *}$} & -2.310 & {$[0.000]^{* * *}$} & -2.371 & {$[0.000]^{* * *}$} \\
\hline No. of observations & 3616 & & 3616 & & 3218 & & 3218 & \\
\hline Pseudo R2 & 0.00113 & & 0.00359 & & 0.00880 & & 0.0174 & \\
\hline chi2 & 4.402 & & 12.40 & & 27.56 & & 48.57 & \\
\hline Unpaid putnamian work & Mean values & & Demographic & controls & Familiar status & & Education & \\
\hline w2 & 0.758 & {$[0.000]^{* * *}$} & 0.755 & {$[0.000]^{* * *}$} & 0.816 & {$[0.000]^{* * *}$} & 0.839 & {$[0.000]^{* * *}$} \\
\hline w4 & 0.834 & {$[0.000]^{* * *}$} & 0.802 & {$[0.000]^{* * *}$} & 0.899 & {$[0.000]^{* * *}$} & 1.031 & {$[0.000]^{* * *}$} \\
\hline Age & & & 0.0859 & {$[0.000]^{* * *}$} & 0.0712 & {$[0.000]^{* * *}$} & 0.0692 & {$[0.000]^{* * *}$} \\
\hline Age2 & & & -0.000834 & {$[0.000]^{* * *}$} & -0.000701 & {$[0.000]^{* * *}$} & -0.00065 & {$[0.001]^{* * *}$} \\
\hline Male & & & 0.0849 & {$[0.358]$} & 0.093 & {$[0.338]$} & 0.0912 & {$[0.348]$} \\
\hline No. of children & & & & & 0.00644 & {$[0.851]$} & 0.0175 & {$[0.614]$} \\
\hline Single & & & & & 0.221 & [0.215] & 0.215 & {$[0.226]$} \\
\hline Married & & & & & 0.385 & {$[0.004]^{* * *}$} & 0.395 & {$[0.003]^{* * *}$} \\
\hline Illiterate & & & & & & & -0.656 & {$[0.001]^{* * *}$} \\
\hline _cons & -2.263 & {$[0.000]^{* * *}$} & -4.23 & {$[0.000]^{* * *}$} & -4.229 & {$[0.000]^{* * *}$} & -4.301 & {$[0.000]^{* * *}$} \\
\hline No. of observations & 3817 & & 3817 & & & 3383 & 3383 & \\
\hline Pseudo R2 & 0.018 & & 0.0282 & & 0.0255 & & 0.0297 & \\
\hline chi2 & 48 & & 88.31 & & 77.03 & & 90.04 & \\
\hline
\end{tabular}


Table A.16 (Continued)

\begin{tabular}{|c|c|c|c|c|c|c|c|c|}
\hline Happiness & Mean values & & Demographi & rols & Familiar status & & Education & \\
\hline w2 & 0.193 & {$[0.025]^{* *}$} & 0.224 & {$[0.010]^{* * *}$} & 0.302 & {$[0.002]^{* * *}$} & 0.309 & {$[0.002]^{* * *}$} \\
\hline w4 & 0.502 & {$[0.000]^{* * *}$} & 0.564 & {$[0.000]^{* * *}$} & 0.701 & {$[0.000]^{* * *}$} & 0.746 & {$[0.000]^{* * *}$} \\
\hline Age & & & -0.0101 & {$[0.335]$} & -0.0737 & {$[0.000]^{* * *}$} & -0.0743 & {$[0.000]^{* * *}$} \\
\hline Age2 & & & -0.0000212 & {$[0.846]$} & 0.000581 & {$[0.000]^{* * *}$} & 0.000597 & {$[0.000]^{* * *}$} \\
\hline Male & & & -0.0537 & {$[0.424]$} & -0.0824 & {$[0.256]$} & -0.0837 & {$[0.249]$} \\
\hline No. of children & & & & & 0.0300 & [0.310] & 0.0334 & {$[0.258]$} \\
\hline Single & & & & & 0.351 & {$[0.012]^{* *}$} & 0.347 & {$[0.013]^{* *}$} \\
\hline Married & & & & & 1.069 & {$[0.000]^{* * *}$} & 1.072 & {$[0.000]^{* * *}$} \\
\hline Illiterate & & & & & & & -0.220 & {$[0.132]$} \\
\hline cut1 & -4.350 & {$[0.000]^{* * *}$} & -4.841 & {$[0.000]^{* * *}$} & -5.533 & {$[0.000]^{* * *}$} & -5.514 & {$[0.000]^{* * *}$} \\
\hline cut2 & -2.186 & {$[0.000]^{* * *}$} & -2.674 & {$[0.000]^{* * *}$} & -3.351 & {$[0.000]^{* * *}$} & -3.332 & {$[0.000]^{* * *}$} \\
\hline cut3 & 1.266 & {$[0.000]^{* * *}$} & 0.807 & {$[0.000]^{* * *}$} & 0.200 & {$[0.503]$} & 0.220 & {$[0.460]$} \\
\hline No. of observations & 3781 & & & & 3358 & & 3358 & \\
\hline Pseudo R2 & 0.00632 & & 0.224 & & 0.0349 & & 0.0353 & \\
\hline chi2 & 40.84 & & & & 187.2 & & 189.0 & \\
\hline
\end{tabular}

p-values in brackets: ${ }^{*} p<0.10,{ }^{* *} p<0.05,{ }^{* * *} p<0.01$.

Table A.17

Logit regression about the trends of relational goods and subjective well-being in Germany.

\begin{tabular}{|c|c|c|c|c|c|c|c|c|}
\hline \multirow{2}{*}{$\begin{array}{l}\text { Germany } \\
\text { Putnam's groups } \\
\text { w2 }\end{array}$} & \multicolumn{2}{|l|}{ Mean values } & \multicolumn{2}{|c|}{ Demographic controls } & \multicolumn{2}{|l|}{ Familiar status } & \multicolumn{2}{|l|}{ Education } \\
\hline & 1.098 & {$[0.000]^{* * *}$} & 1.100 & {$[0.000]^{* * *}$} & 0.948 & {$[0.000]^{* * *}$} & 0.948 & {$[0.000]^{* * *}$} \\
\hline w4 & 0.522 & {$[0.000]^{* * *}$} & 0.535 & {$[0.000]^{* * *}$} & 0.366 & {$[0.000]^{* * *}$} & 0.369 & {$[0.000]^{* * *}$} \\
\hline Age & & & 0.0227 & {$[0.005]^{* * *}$} & 0.0184 & {$[0.063]^{*}$} & 0.0183 & {$[0.065]^{*}$} \\
\hline Age2 & & & -0.000258 & {$[0.002]^{* * *}$} & -0.000215 & {$[0.027]^{* *}$} & -0.000214 & {$[0.028]^{* *}$} \\
\hline Male & & & 0.176 & {$[0.001]^{* * *}$} & 0.179 & {$[0.001]^{* * *}$} & 0.179 & {$[0.001]^{* * *}$} \\
\hline No. of children & & & & & 0.0462 & {$[0.063]^{*}$} & 0.0465 & {$[0.062]^{*}$} \\
\hline Single & & & & & 0.398 & {$[0.000]^{* * *}$} & 0.397 & {$[0.000]^{* * *}$} \\
\hline Married & & & & & 0.144 & {$[0.038]^{* *}$} & 0.143 & {$[0.040]^{* *}$} \\
\hline Illiterate & & & & & & & -0.153 & {$[0.663]$} \\
\hline _cons & -1.031 & {$[0.000]^{* * *}$} & -1.534 & {$[0.000]^{* * *}$} & -1.516 & {$[0.000]^{* * *}$} & -1.514 & {$[0.000]^{* * *}$} \\
\hline No. of observations & 6778 & & 6775 & & 6230 & & 6230 & \\
\hline Pseudo R2 & 0.0309 & & 0.0336 & & 0.0271 & & 0.0271 & \\
\hline chi2 & 271.2 & & 290.5 & & 218.7 & & 218.7 & \\
\hline Olson's groups & Mean values & & Demographic c & controls & Familiar status & & Education & \\
\hline w2 & 0.831 & {$[0.000]^{* * *}$} & 0.848 & {$[0.000]^{* * *}$} & 0.886 & {$[0.000]^{* * *}$} & 0.886 & {$[0.000]^{* * *}$} \\
\hline w4 & -0.673 & {$[0.000]^{* * *}$} & -0.677 & {$[0.000]^{* * *}$} & -0.610 & {$[0.000]^{* * *}$} & -0.593 & {$[0.000]^{* * *}$} \\
\hline Age & & & 0.109 & {$[0.000]^{* * *}$} & 0.0755 & {$[0.000]^{* * *}$} & 0.0754 & {$[0.000]^{* * *}$} \\
\hline Age2 & & & -0.00120 & {$[0.000]^{* * *}$} & -0.000889 & {$[0.000]^{* * *}$} & -0.000889 & {$[0.000]^{* * *}$} \\
\hline Male & & & 0.747 & {$[0.000]^{* * *}$} & 0.742 & {$[0.000]^{* * *}$} & 0.743 & {$[0.000]^{* * *}$} \\
\hline No. of children & & & & & 0.000739 & {$[0.979]$} & 0.00188 & {$[0.947]$} \\
\hline Single & & & & & -0.248 & {$[0.041]^{* *}$} & -0.252 & {$[0.039]^{* *}$} \\
\hline Married & & & & & 0.185 & {$[0.023]^{* *}$} & 0.181 & {$[0.026]^{* *}$} \\
\hline Illiterate & & & & & & & -1.705 & {$[0.097]^{*}$} \\
\hline _cons & -1.240 & {$[0.000]^{* * *}$} & -3.761 & {$[0.000]^{* * *}$} & -3.060 & {$[0.000]^{* * *}$} & -3.057 & {$[0.000]^{* * *}$} \\
\hline No. of observations & 6778 & & 6775 & & 6230 & & 6230 & \\
\hline Pseudo R2 & 0.0637 & & 0.105 & & 0.109 & & 0.109 & \\
\hline chi2 & 451.8 & & 728.4 & & 689.3 & & 685.4 & \\
\hline Other groups & Mean values & & Demographic co & ontrols & Familiar status & & Education & \\
\hline w2 & 0.794 & {$[0.000]^{* * *}$} & 0.797 & {$[0.000]^{* * *}$} & 1.342 & {$[0.000]^{* * *}$} & 1.342 & {$[0.000]^{* * *}$} \\
\hline w4 & -0.111 & [0.307] & -0.0946 & {$[0.376]$} & 0.416 & {$[0.005]^{* * *}$} & 0.409 & {$[0.006]^{* * *}$} \\
\hline Age & & & 0.00730 & [0.483] & 0.0306 & {$[0.018]^{* *}$} & 0.0308 & {$[0.018]^{* *}$} \\
\hline Age2 & & & -0.0000934 & [0.383] & -0.000303 & {$[0.018]^{* *}$} & -0.000305 & {$[0.018]^{* *}$} \\
\hline Male & & & 0.192 & {$[0.003]^{* * *}$} & 0.0848 & {$[0.217]$} & 0.0845 & {$[0.219]$} \\
\hline No. of children & & & & & 0.125 & {$[0.000]^{* * *}$} & 0.125 & {$[0.000]^{* * *}$} \\
\hline Single & & & & & 0.403 & {$[0.002]^{* * *}$} & 0.405 & {$[0.002]^{* * *}$} \\
\hline Married & & & & & 0.188 & {$[0.042]^{* *}$} & 0.190 & {$[0.040]^{* *}$} \\
\hline Illiterate & & & & & & & 0.337 & {$[0.442]$} \\
\hline _cons & -1.940 & {$[0.000]^{* * *}$} & -2.147 & {$[0.000]^{* * *}$} & -3.576 & {$[0.000]^{* * *}$} & -3.580 & {$[0.000]^{* * *}$} \\
\hline No. of observations & 6778 & & 6775 & & 6230 & & 6230 & \\
\hline Pseudo R2 & 0.0276 & & 0.0292 & & 0.0408 & & 0.0409 & \\
\hline chi2 & 166.7 & & 180.2 & & 223.7 & & 223.6 & \\
\hline Trust in others & Mean values & & Demographic contr & ols & Familiar status & & Education & \\
\hline w2 & 0.0282 & [0.711] & 0.0571 & {$[0.454]$} & 0.148 & [0.128] & 0.148 & [0.128] \\
\hline w3 & 0.0451 & {$[0.576]$} & 0.0606 & {$[0.454]$} & 0.144 & [0.153] & 0.160 & {$[0.115]$} \\
\hline w4 & 0.231 & {$[0.004]^{* * *}$} & 0.290 & {$[0.000]^{* * *}$} & 0.359 & {$[0.000]^{* * *}$} & 0.368 & {$[0.000]^{* * *}$} \\
\hline Age & & & -0.00253 & {$[0.751]$} & 0.0161 & {$[0.096]^{*}$} & 0.0159 & {$[0.101]$} \\
\hline Age2 & & & -0.0000578 & {$[0.484]$} & -0.000205 & {$[0.034]^{* *}$} & -0.000200 & {$[0.038]^{* *}$} \\
\hline Male & & & 0.0801 & {$[0.095]^{*}$} & 0.0469 & [0.353] & 0.0455 & [0.367] \\
\hline No. of children & & & & & -0.00283 & [0.896] & -0.00319 & [0.883] \\
\hline
\end{tabular}


Table A.17 (Continued)

\begin{tabular}{|c|c|c|c|c|c|c|c|c|}
\hline Trust in others & Mean values & & Demographic con & trols & Familiar status & & Education & \\
\hline Single & & & & & 0.334 & {$[0.000]^{* * *}$} & 0.329 & {$[0.000]^{* * *}$} \\
\hline Married & & & & & 0.0976 & {$[0.132]$} & 0.0937 & {$[0.148]$} \\
\hline Illiterate & & & & & & & -0.556 & {$[0.023]^{* *}$} \\
\hline _cons & -0.741 & {$[0.000]^{* * *}$} & -0.558 & {$[0.002]^{* * *}$} & -1.246 & {$[0.000]^{* * *}$} & -1.242 & {$[0.000]^{* * *}$} \\
\hline No. of observations & 7870 & & 7861 & & 7408 & & 7408 & \\
\hline Pseudo R2 & 0.00139 & & 0.00510 & & 0.00636 & & 0.00695 & \\
\hline chi2 & 14.13 & & 51.16 & & 59.29 & & 64.36 & \\
\hline Unpaid putnamian work & Mean values & & Demographi & ic controls & Familiar status & & Education & \\
\hline w2 & 0.662 & {$[0.000]^{* * *}$} & 0.658 & {$[0.000]^{* * *}$} & 0.671 & {$[0.000]^{* * *}$} & 0.672 & {$[0.000]^{* * *}$} \\
\hline w4 & 0.193 & {$[0.100]$} & 0.173 & {$[0.141]$} & 0.157 & {$[0.230]$} & 0.171 & [0.192] \\
\hline Age & & & 0.0479 & {$[0.000]^{* * *}$} & 0.0347 & {$[0.007]^{* * *}$} & 0.0342 & {$[0.008]^{* * *}$} \\
\hline Age2 & & & -0.000498 & {$[0.000]^{* * *}$} & -0.000387 & {$[0.002]^{* * *}$} & -0.000381 & {$[0.003]^{* * *}$} \\
\hline Male & & & 0.319 & {$[0.000]^{* * *}$} & 0.335 & {$[0.000]^{* * *}$} & 0.337 & {$[0.000]^{* * *}$} \\
\hline No. of children & & & & & 0.067 & {$[0.046]^{* *}$} & 0.0682 & {$[0.042]^{* *}$} \\
\hline Single & & & & & 0.179 & [0.209] & 0.177 & {$[0.215]$} \\
\hline Married & & & & & 0.226 & {$[0.020]^{* *}$} & 0.225 & {$[0.020]^{* *}$} \\
\hline Illiterate & & & & & & & -1.122 & {$[0.276]$} \\
\hline _cons & -1.794 & {$[0.000]^{* * *}$} & -2.942 & {$[0.000]^{* * *}$} & -2.891 & {$[0.000]^{* * *}$} & -2.883 & {$[0.000]^{* * *}$} \\
\hline No. of observations & 6778 & & 6775 & & 6230 & & 6230 & \\
\hline Pseudo R2 & 0.012 & & 0.0201 & & 0.0216 & & 0.0221 & \\
\hline chi2 & 60.86 & & 101 & & 107.8 & & 108.3 & \\
\hline Happiness & Mean values & & Demographic co & ontrols & Familiar status & & Education & \\
\hline w2 & 0.142 & {$[0.018]^{* *}$} & 0.171 & {$[0.004]^{* * *}$} & 0.163 & {$[0.034]^{* *}$} & 0.164 & {$[0.033]^{* *}$} \\
\hline W3 & 0.0576 & {$[0.423]$} & 0.0818 & [0.259] & 0.122 & {$[0.158]$} & 0.142 & {$[0.102]$} \\
\hline w4 & 0.0565 & [0.435] & 0.115 & [0.115] & 0.147 & {$[0.090]^{*}$} & 0.159 & {$[0.067]^{*}$} \\
\hline Age & & & -0.0121 & {$[0.106]$} & 0.0726 & {$[0.000]^{* * *}$} & -0.0732 & {$[0.000]^{* * *}$} \\
\hline Age2 & & & 0.0000524 & [0.513] & 0.000654 & {$[0.000]^{* * *}$} & 0.000663 & {$[0.000]^{* * *}$} \\
\hline Male & & & 0.0340 & {$[0.460]$} & 0.0362 & {$[0.450]$} & -0.0393 & {$[0.413]$} \\
\hline No. of children & & & & & -0.0326 & {$[0.140]$} & -0.0331 & {$[0.134]$} \\
\hline Single & & & & & 0.0526 & [0.553] & 0.0469 & {$[0.596]$} \\
\hline Married & & & & & 0.938 & {$[0.000]^{* * *}$} & 0.935 & {$[0.000]^{* * *}$} \\
\hline Illiterate & & & & & & & -0.632 & {$[0.009]^{* * *}$} \\
\hline cut1 & -3.876 & {$[0.000]^{* * *}$} & -4.258 & {$[0.000]^{* * *}$} & -5.160 & {$[0.000]^{* * *}$} & -5.173 & {$[0.000]^{* * *}$} \\
\hline cut2 & -1.590 & {$[0.000]^{* * *}$} & -1.968 & {$[0.000]^{* * *}$} & -2.840 & {$[0.000]^{* * *}$} & -2.851 & {$[0.000]^{* * *}$} \\
\hline cut3 & 1.744 & {$[0.000]^{* * *}$} & 1.375 & {$[0.000]^{* * *}$} & 0.549 & {$[0.015]^{* *}$} & 0.541 & {$[0.017]^{* *}$} \\
\hline No. of observations & 8409 & & 8400 & & 7887 & & 7887 & \\
\hline Pseudo R2 & 0.000335 & & 0.00228 & & 0.0216 & & 0.0221 & \\
\hline chi2 & 6.269 & & 36.37 & & 303.3 & & 309.5 & \\
\hline
\end{tabular}

$p$-values in brackets: ${ }^{*} p<0.10,{ }^{* *} p<0.05,{ }^{* * *} p<0.01$.

Table A.18

Logit regression about the trends of relational goods and subjective well-being in Netherlands.

\begin{tabular}{|c|c|c|c|c|c|c|c|c|}
\hline \multirow{2}{*}{$\begin{array}{l}\text { Netherlands } \\
\text { Putnam's groups }\end{array}$} & \multicolumn{2}{|l|}{ Mean values } & \multicolumn{2}{|c|}{ Demographic controls } & \multicolumn{2}{|l|}{ Familiar status } & \multicolumn{2}{|l|}{ Education } \\
\hline & 1.308 & {$[0.000]^{* * *}$} & 1.259 & {$[0.000]^{* * *}$} & 1.239 & {$[0.000]^{* * *}$} & 1.240 & {$[0.000]^{* * *}$} \\
\hline w4 & 2.088 & {$[0.000]^{* * *}$} & 2.003 & {$[0.000]^{* * *}$} & 1.986 & {$[0.000]^{* * *}$} & 2.005 & {$[0.000]^{* * *}$} \\
\hline Age & & & 0.0572 & {$[0.000]^{* * *}$} & 0.0282 & {$[0.133]$} & 0.0256 & {$[0.166]$} \\
\hline Age2 & & & -0.000541 & {$[0.000]^{* * *}$} & -0.000277 & {$[0.134]$} & -0.000247 & {$[0.172]$} \\
\hline Male & & & 0.0390 & {$[0.644]$} & 0.169 & {$[0.082]^{*}$} & 0.164 & {$[0.092]^{*}$} \\
\hline No. of children & & & & & 0.0409 & {$[0.326]$} & 0.0408 & {$[0.328]$} \\
\hline Single & & & & & 0.336 & {$[0.068]^{*}$} & 0.326 & {$[0.077]^{*}$} \\
\hline Married & & & & & 0.490 & {$[0.000]^{* * *}$} & 0.490 & {$[0.000]^{* * *}$} \\
\hline Illiterate & & & & & & & -1.130 & {$[0.106]$} \\
\hline _coris & -0.0442 & {$[0.440]$} & -1.314 & {$[0.000]^{* * *}$} & -1.070 & {$[0.015]^{* *}$} & -1.018 & {$[0.020]^{* *}$} \\
\hline No. of observations & 3241 & & 3217 & & 2744 & & 2744 & \\
\hline Pseudo R2 & 0.118 & & 0.123 & & 0.0978 & & 0.0985 & \\
\hline chi2 & 408.3 & & 416.9 & & 274.8 & & 275.8 & \\
\hline Olson's groups & Mean values & & Demographic & ntrols & Familiar status & & Education & \\
\hline w2 & 0.682 & {$[0.000]^{* * *}$} & 0.590 & {$[0.000]^{* * *}$} & 0.728 & {$[0.000]^{* * *}$} & 0.728 & {$[0.000]^{* * *}$} \\
\hline w4 & 0.783 & {$[0.000]^{* * *}$} & 0.612 & {$[0.000]^{* * *}$} & 0.737 & {$[0.000]^{* * *}$} & 0.739 & {$[0.000]^{* * *}$} \\
\hline Age & & & 0.130 & {$[0.000]^{* * *}$} & 0.121 & {$[0.000]^{* * *}$} & 0.121 & {$[0.000]^{* * *}$} \\
\hline Age2 & & & -0.00131 & {$[0.000]^{* * *}$} & -0.00118 & {$[0.000]^{* * *}$} & -0.00118 & {$[0.000]^{* * *}$} \\
\hline Male & & & 0.569 & {$[0.000]^{* * *}$} & 0.542 & {$[0.000]^{* * *}$} & 0.541 & {$[0.000]^{* * *}$} \\
\hline No. of children & & & & & -0.0393 & {$[0.272]$} & -0.0396 & {$[0.268]$} \\
\hline Single & & & & & 0.333 & {$[0.041]^{* *}$} & 0.331 & {$[0.042]^{* *}$} \\
\hline Married & & & & & 0.468 & {$[0.000]^{* * *}$} & 0.468 & {$[0.000]^{* * *}$} \\
\hline Illiterate & & & & & & & -0.317 & {$[0.691]$} \\
\hline _cons & -1.078 & {$[0.000]^{* * *}$} & -4.091 & {$[0.000]^{* * *}$} & -4.377 & {$[0.000]^{* * *}$} & -4.369 & {$[0.000]^{* * *}$} \\
\hline No. of observations & 3241 & & 3217 & & 2744 & & 2744 & \\
\hline Pseudo R2 & 0.0212 & & 0.0571 & & 0.0525 & & 0.0526 & \\
\hline chi2 & 85.46 & & 221.9 & & 183.7 & & 184.5 & \\
\hline
\end{tabular}


Table A.18 (Continued)

\begin{tabular}{|c|c|c|c|c|c|c|c|c|}
\hline Other groups & Mean values & & Demographic con & trols & Familiar status & & Education & \\
\hline w2 & 0.835 & {$[0.000]^{* * *}$} & 0.801 & {$[0.000]^{* * *}$} & 0.881 & {$[0.000]^{* * *}$} & 0.881 & {$[0.000]^{* * *}$} \\
\hline w4 & 0.733 & {$[0.000]^{* * *}$} & 0.676 & {$[0.000]^{* * *}$} & 0.733 & {$[0.000]^{* * *}$} & 0.730 & {$[0.000]^{* * *}$} \\
\hline Age & & & 0.00196 & [0.889] & 0.0271 & {$[0.121]$} & 0.0278 & {$[0.111]$} \\
\hline Age2 & & & 0.0000656 & {$[0.642]$} & -0.000143 & {$[0.401]$} & -0.000150 & {$[0.374]$} \\
\hline Male & & & -0.0123 & [0.886] & -0.0510 & {$[0.585]$} & -0.0497 & [0.595] \\
\hline No. of children & & & & & 0.0410 & {$[0.276]$} & 0.0412 & {$[0.274]$} \\
\hline Single & & & & & 0.609 & {$[0.001]^{* * *}$} & 0.612 & {$[0.001]^{* * *}$} \\
\hline Married & & & & & 0.402 & {$[0.003]^{* * *}$} & 0.403 & {$[0.003]^{* * *}$} \\
\hline Illiterate & & & & & & & 0.268 & {$[0.704]$} \\
\hline _cons & -1.788 & {$[0.000]^{* * *}$} & -1.978 & {$[0.000]^{* * *}$} & -3.137 & {$[0.000]^{* * *}$} & -3.151 & {$[0.000]^{* * *}$} \\
\hline No. of observations & 3241 & & 3217 & & 2744 & & 2744 & \\
\hline Pseudo R2 & 0.0214 & & 0.0241 & & 0.0257 & & 0.0257 & \\
\hline chi2 & 68.11 & & 72.77 & & 67.71 & & 67.90 & \\
\hline Trust in others & Mean values & & Demographic con & trols & Familiar status & & Education & \\
\hline w2 & 0.349 & {$[0.000]^{* * *}$} & 0.417 & {$[0.000]^{* * *}$} & 0.401 & {$[0.000]^{* * *}$} & 0.401 & {$[0.000]^{* * *}$} \\
\hline W4 & 0.619 & {$[0.000]^{* * *}$} & 0.740 & {$[0.000]^{* * *}$} & 0.678 & {$[0.000]^{* * *}$} & 0.682 & {$[0.000]^{* * *}$} \\
\hline Age & & & 0.000297 & {$[0.980]$} & 0.0174 & {$[0.272]$} & 0.0166 & {$[0.297]$} \\
\hline Age2 & & & -0.000186 & [0.133] & -0.000319 & {$[0.040]^{* *}$} & -0.000310 & {$[0.048]^{* *}$} \\
\hline Male & & & 0.135 & {$[0.073]^{*}$} & 0.0762 & [0.359] & 0.0743 & {$[0.371]$} \\
\hline No. of children & & & & & 0.0269 & {$[0.456]$} & 0.0265 & {$[0.462]$} \\
\hline Single & & & & & 0.647 & {$[0.000]^{* * *}$} & 0.644 & {$[0.000]^{* * *}$} \\
\hline Married & & & & & 0.271 & {$[0.017]^{* *}$} & 0.271 & {$[0.017]^{* *}$} \\
\hline Illiterate & & & & & & & -0.435 & {$[0.561]$} \\
\hline cons & -0.210 & {$[0.001]^{* * *}$} & 0.0488 & [0.852] & -0.694 & {$[0.070]^{*}$} & -0.677 & {$[0.079]^{*}$} \\
\hline No. of observations & 3034 & & 3014 & & 2596 & & 2596 & \\
\hline Pseudo R2 & 0.0117 & & 0.0274 & & 0.0335 & & 0.0336 & \\
\hline chi2 & 48.58 & & 105.6 & & 111.2 & & 111.3 & \\
\hline Unpaid putnamian work & Mean values & & Demographic & controls & Familiar status & & Education & \\
\hline w2 & 0.556 & {$[0.000]^{* * *}$} & 0.571 & {$[0.000]^{* * *}$} & 0.48 & {$[0.000]^{* * *}$} & 0.48 & {$[0.000]^{* * *}$} \\
\hline w4 & 1.162 & {$[0.000]^{* * *}$} & 1.164 & {$[0.000]^{* * *}$} & 1.074 & {$[0.000]^{* * *}$} & 1.084 & {$[0.000]^{* * *}$} \\
\hline Age & & & 0.0779 & {$[0.000]^{* * *}$} & 0.0556 & {$[0.002]^{* * *}$} & 0.0537 & {$[0.003]^{* * *}$} \\
\hline Age2 & & & -0.000765 & {$[0.000]^{* * *}$} & -0.000551 & {$[0.001]^{* * *}$} & -0.000529 & {$[0.003]^{* * *}$} \\
\hline Male & & & 0.0266 & {$[0.752]$} & 0.0345 & [0.698] & 0.0306 & {$[0.731]$} \\
\hline No. of children & & & & & 0.0504 & {$[0.182]$} & 0.0501 & {$[0.185]$} \\
\hline Single & & & & & 0.479 & {$[0.005]^{* * *}$} & 0.471 & {$[0.006]^{* * *}$} \\
\hline Married & & & & & 0.438 & {$[0.001]^{* * *}$} & 0.436 & {$[0.001]^{* * *}$} \\
\hline Illiterate & & & & & & & -1.118 & [0.163] \\
\hline _cons & -1.434 & {$[0.000]^{* * *}$} & -3.185 & {$[0.000]^{* * *}$} & -3.037 & {$[0.000]^{* * *}$} & -3 & {$[0.000]^{* * *}$} \\
\hline No. of observations & 3241 & & 3217 & & 2744 & & 2744 & \\
\hline Pseudo R2 & 0.0384 & & 0.0484 & & 0.0376 & & 0.0382 & \\
\hline chi2 & 125.1 & & 183.1 & & 113.3 & & 116.8 & \\
\hline Happiness & Mean values & & Demographic cor & trols & Familiar status & & Education & \\
\hline w2 & 0.373 & {$[0.000]^{* * *}$} & 0.397 & {$[0.000]^{* * *}$} & 0.475 & {$[0.000]^{* * *}$} & 0.476 & {$[0.000]^{* * *}$} \\
\hline W4 & 0.391 & {$[0.000]^{* * *}$} & 0.445 & {$[0.000]^{* * *}$} & 0.600 & {$[0.000]^{* * *}$} & 0.607 & {$[0.000]^{* * *}$} \\
\hline Age & & & 0.00717 & [0.527] & -0.0896 & {$[0.000]^{* * *}$} & -0.0916 & {$[0.000]^{* * *}$} \\
\hline Age2 & & & -0.000156 & [0.195] & 0.000767 & {$[0.000]^{* * *}$} & 0.000790 & {$[0.000]^{* * *}$} \\
\hline Male & & & -0.168 & {$[0.017]^{* *}$} & -0.118 & {$[0.138]$} & -0.120 & {$[0.130]$} \\
\hline No. of children & & & & & -0.0115 & {$[0.742]$} & -0.0116 & {$[0.741]$} \\
\hline Single & & & & & 0.0568 & {$[0.723]$} & 0.0521 & {$[0.746]$} \\
\hline Married & & & & & 1.157 & {$[0.000]^{* * *}$} & 1.158 & {$[0.000]^{* * *}$} \\
\hline Illiterate & & & & & & & -0.944 & {$[0.468]$} \\
\hline cut1 & -4.802 & {$[0.000]^{* * *}$} & -4.878 & {$[0.000]^{* * *}$} & -6.226 & {$[0.000]^{* * *}$} & -6.267 & {$[0.000]^{* * *}$} \\
\hline cut2 & -2.811 & {$[0.000]^{* * *}$} & -2.892 & {$[0.000]^{* * *}$} & -4.282 & {$[0.000]^{* * *}$} & -4.322 & {$[0.000]^{* * *}$} \\
\hline cut3 & 0.582 & {$[0.000]^{* * *}$} & 0.515 & {$[0.032]^{* *}$} & -0.928 & {$[0.014]^{* *}$} & -0.967 & {$[0.012]^{* *}$} \\
\hline No. of observations & 3212 & & & & 2733 & & 2733 & \\
\hline Pseudo R2 & 0.00513 & & 0.397 & & 0.0410 & & 0.0413 & \\
\hline chi2 & 29.24 & & & & 173.3 & & 173.8 & \\
\hline
\end{tabular}

p-values in brackets: ${ }^{*} p<0.10,{ }^{* *} p<0.05,{ }^{* * *} p<0.01$. 
Table A.19

Logit regression about the trends of relational goods and subjective well-being in Belgium.

\begin{tabular}{|c|c|c|c|c|c|c|c|c|}
\hline \multirow{2}{*}{$\begin{array}{l}\begin{array}{l}\text { Belgium } \\
\text { Putnam's groups }\end{array} \\
\text { w2 }\end{array}$} & \multicolumn{2}{|l|}{ Mean values } & \multicolumn{2}{|c|}{ Demographic controls } & \multicolumn{2}{|l|}{ Familiar status } & \multicolumn{2}{|l|}{ Education } \\
\hline & 1.010 & {$[0.000]^{* * *}$} & 1.005 & {$[0.000]^{* * *}$} & 0.926 & {$[0.000]^{* * *}$} & 0.927 & {$[0.000]^{* * *}$} \\
\hline w4 & 1.166 & {$[0.000]^{* * *}$} & 1.181 & {$[0.000]^{* * *}$} & 1.096 & {$[0.000]^{* * *}$} & 1.110 & {$[0.000]^{* * *}$} \\
\hline Age & & & 0.0149 & {$[0.078]^{*}$} & 0.0190 & {$[0.066]^{*}$} & 0.0184 & {$[0.076]^{*}$} \\
\hline Age2 & & & -0.000222 & {$[0.012]^{* *}$} & -0.000265 & {$[0.010]^{* * *}$} & -0.000256 & {$[0.013]^{* *}$} \\
\hline Male & & & 0.272 & {$[0.000]^{* * *}$} & 0.282 & {$[0.000]^{* * *}$} & 0.282 & {$[0.000]^{* * *}$} \\
\hline No. of children & & & & & 0.0699 & {$[0.002]^{* * *}$} & 0.0710 & {$[0.002]^{* * *}$} \\
\hline Single & & & & & 0.422 & {$[0.000]^{* * *}$} & 0.414 & {$[0.000]^{* * *}$} \\
\hline Married & & & & & 0.119 & {$[0.124]$} & 0.111 & {$[0.151]$} \\
\hline Illiterate & & & & & & & -0.680 & {$[0.055]^{*}$} \\
\hline _cons & -1.235 & {$[0.000]^{* * *}$} & -1.523 & {$[0.000]^{* * *}$} & -1.803 & {$[0.000]^{* * *}$} & -1.790 & {$[0.000]^{* * *}$} \\
\hline No. of observations & 5849 & & 5842 & & 5340 & & 5340 & \\
\hline Pseudo R2 & 0.0288 & & 0.0350 & & 0.0292 & & 0.0298 & \\
\hline chi2 & 203.6 & & 234.8 & & 186.1 & & 189.4 & \\
\hline Olson's groups & Mean values & & Demographic con & trols & Familiar status & & Education & \\
\hline w2 & 0.272 & {$[0.002]^{* * *}$} & 0.203 & {$[0.021]^{* *}$} & 0.240 & {$[0.023]^{* *}$} & 0.240 & {$[0.023]^{* *}$} \\
\hline w4 & 0.435 & {$[0.000]^{* * *}$} & 0.390 & {$[0.000]^{* * *}$} & 0.463 & {$[0.000]^{* * *}$} & 0.469 & {$[0.000]^{* * *}$} \\
\hline Age & & & 0.0987 & {$[0.000]^{* * *}$} & 0.0623 & {$[0.000]^{* * *}$} & 0.0619 & {$[0.000]^{* * *}$} \\
\hline ase 2 & & & -0.00114 & {$[0.000]^{* * *}$} & -0.000804 & {$[0.000]^{* * *}$} & -0.000799 & {$[0.000]^{* * *}$} \\
\hline Male & & & 0.622 & {$[0.000]^{* * *}$} & 0.661 & {$[0.000]^{* * *}$} & 0.661 & {$[0.000]^{* * *}$} \\
\hline No. of children & & & & & 0.0408 & {$[0.113]$} & 0.0413 & {$[0.109]$} \\
\hline Single & & & & & -0.210 & [0.116] & -0.214 & [0.109] \\
\hline Married & & & & & 0.165 & {$[0.072]^{*}$} & 0.161 & {$[0.079]^{*}$} \\
\hline Illiterate & & & & & & & -0.344 & {$[0.419]$} \\
\hline _cons & -1.419 & {$[0.000]^{* * *}$} & -3.502 & {$[0.000]^{* * *}$} & 2.832 & {$[0.000]^{* * *}$} & -2.825 & {$[0.000]^{* * *}$} \\
\hline No. of observations & 5849 & & 5842 & & 5340 & & 5340 & \\
\hline Pseudo R2 & 0.00367 & & 0.0383 & & 0.0397 & & 0.0399 & \\
\hline chi2 & 23.15 & & 203.7 & & 205.1 & & 205.4 & \\
\hline Other groups & Mean values & & Demographic cont & trols & Familiar status & & Education & \\
\hline w2 & 1.332 & {$[0.000]^{* * *}$} & 1.342 & {$[0.000]^{* * *}$} & 1.726 & {$[0.000]^{* * *}$} & 1.727 & {$[0.000]^{* * *}$} \\
\hline w4 & 1.542 & {$[0.000]^{* * *}$} & 1.551 & {$[0.000]^{* * *}$} & 1.908 & {$[0.000]^{* * *}$} & 1.919 & {$[0.000]^{* * *}$} \\
\hline Age & & & -0.00634 & {$[0.522]$} & 0.00195 & {$[0.868]$} & 0.00143 & {$[0.903]$} \\
\hline Age2 & & & 0.0000524 & {$[0.603]$} & -0.0000231 & {$[0.841]$} & -0.0000164 & {$[0.887]$} \\
\hline Male & & & -0.138 & {$[0.031]^{* *}$} & -0.166 & {$[0.013]^{* *}$} & -0.166 & {$[0.013]^{* *}$} \\
\hline No. of children & & & & & 0.147 & {$[0.000]^{* * *}$} & 0.148 & {$[0.000]^{* * *}$} \\
\hline Single & & & & & 0.486 & {$[0.000]^{* * *}$} & 0.480 & {$[0.000]^{* * *}$} \\
\hline Married & & & & & 0.164 & {$[0.069]^{*}$} & 0.157 & {$[0.082]^{*}$} \\
\hline Illiterate & & & & & & & -0.549 & {$[0.188]$} \\
\hline _cons & -2.461 & {$[0.000]^{* * *}$} & -2.242 & {$[0.000]^{* * *}$} & -3.245 & {$[0.000]^{* * *}$} & -3.235 & {$[0.000]^{* * *}$} \\
\hline No. of observations & 5849 & & 5842 & & 5340 & & 5340 & \\
\hline Pseudo R2 & 0.0351 & & 0.0360 & & 0.0400 & & 0.0403 & \\
\hline chi2 & 163.4 & & 171.9 & & 155.6 & & 157.5 & \\
\hline Trust in others & Mean values & & Demographic contr & rols & Familiar status & & Education & \\
\hline w2 & 0.202 & {$[0.013]^{* *}$} & 0.202 & {$[0.013]^{* *}$} & 0.160 & [0.108] & 0.161 & {$[0.108]$} \\
\hline W4 & 0.00245 & {$[0.977]$} & 0.0161 & {$[0.853]$} & -0.0469 & [0.655] & -0.0397 & {$[0.706]$} \\
\hline Age & & & 0.00396 & {$[0.673]$} & 0.0115 & {$[0.315]$} & 0.0111 & {$[0.331]$} \\
\hline Age2 & & & -0.0000958 & {$[0.326]$} & -0.000147 & [0.199] & -0.000142 & {$[0.214]$} \\
\hline Male & & & 0.157 & {$[0.008]^{* * *}$} & 0.140 & {$[0.024]^{* *}$} & 0.141 & {$[0.023]^{* *}$} \\
\hline No. of children & & & & & 0.0323 & {$[0.176]$} & 0.0327 & {$[0.172]$} \\
\hline Single & & & & & 0.302 & {$[0.010]^{* *}$} & 0.297 & {$[0.011]^{* *}$} \\
\hline Married & & & & & 0.117 & {$[0.164]$} & 0.113 & {$[0.180]$} \\
\hline Illiterate & & & & & & & -0.363 & {$[0.370]$} \\
\hline _cons & -0.887 & {$[0.000]^{* * *}$} & -0.922 & {$[0.000]^{* * *}$} & -1.271 & {$[0.000]^{* * *}$} & -1.263 & {$[0.000]^{* * *}$} \\
\hline No. of observations & 5401 & & 5395 & & 4958 & & 4958 & \\
\hline Pseudo R2 & 0.00173 & & 0.00440 & & 0.00489 & & 0.00503 & \\
\hline chi2 & 11.57 & & 29.79 & & 29.89 & & 30.45 & \\
\hline Unpaid putnamian work & Mean values & & Demographic & controls & Familiar status & & Education & \\
\hline w2 & 0.452 & {$[0.000]^{* * *}$} & 0.434 & {$[0.000]^{* * *}$} & 0.469 & {$[0.000]^{* * *}$} & 0.47 & {$[0.000]^{* * *}$} \\
\hline w4 & 0.685 & {$[0.000]^{* * *}$} & 0.676 & {$[0.000]^{* * *}$} & 0.683 & {$[0.000]^{* * *}$} & 0.688 & {$[0.000]^{* * *}$} \\
\hline Age & & & 0.0336 & {$[0.005]^{* * *}$} & 0.0507 & {$[0.001]^{* * *}$} & 0.0505 & {$[0.001]^{* * *}$} \\
\hline Age2 & & & -0.000369 & {$[0.003]^{* * *}$} & -0.00053 & {$[0.000]^{* * *}$} & -0.000527 & {$[0.000]^{* * *}$} \\
\hline Male & & & 0.269 & {$[0.000]^{* * *}$} & 0.245 & {$[0.001]^{* * *}$} & 0.245 & {$[0.001]^{* * *}$} \\
\hline No. of children & & & & & 0.0995 & {$[0.001]^{* * *}$} & 0.0998 & {$[0.001]^{* * *}$} \\
\hline Single & & & & & 0.624 & {$[0.000]^{* * *}$} & 0.621 & {$[0.000]^{* * *}$} \\
\hline Married & & & & & 0.129 & {$[0.227]$} & 0.125 & {$[0.239]$} \\
\hline Illiterate & & & & & & & -0.224 & {$[0.641]$} \\
\hline _cons & -1.704 & {$[0.000]^{* * *}$} & -2.481 & {$[0.000]^{* * *}$} & -3.261 & {$[0.000]^{* * *}$} & -3.257 & {$[0.000]^{* * *}$} \\
\hline No. of observations & 5849 & & 5842 & & 5340 & & 5340 & \\
\hline Pseudo R2 & 0.00857 & & 0.0138 & & 0.0181 & & 0.0182 & \\
\hline chi2 & 44.84 & & 69.84 & & 81.55 & & 81.64 & \\
\hline
\end{tabular}


Table A.19 (Continued)

\begin{tabular}{|c|c|c|c|c|c|c|c|c|}
\hline Happiness & Mean values & & Demographi & rols & Familiar status & & Education & \\
\hline w2 & 0.207 & {$[0.002]^{* * *}$} & 0.223 & {$[0.001]^{* * *}$} & 0.352 & {$[0.000]^{* * *}$} & 0.352 & {$[0.000]^{* * *}$} \\
\hline W4 & 0.231 & {$[0.001]^{* * *}$} & 0.266 & {$[0.000]^{* * *}$} & 0.403 & {$[0.000]^{* * *}$} & 0.395 & {$[0.000]^{* * *}$} \\
\hline Age & & & -0.00815 & {$[0.306]$} & -0.0634 & {$[0.000]^{* * *}$} & -0.0630 & {$[0.000]^{* * *}$} \\
\hline Age2 & & & -0.0000125 & [0.881] & 0.000526 & {$[0.000]^{* * *}$} & 0.000520 & {$[0.000]^{* * *}$} \\
\hline Male & & & -0.00316 & [0.952] & -0.0590 & {$[0.289]$} & -0.0597 & {$[0.284]$} \\
\hline No. of children & & & & & 0.0256 & {$[0.251]$} & 0.0252 & {$[0.260]$} \\
\hline Single & & & & & 0.241 & {$[0.037]^{* *}$} & 0.247 & {$[0.033]^{* *}$} \\
\hline Married & & & & & 1.064 & {$[0.000]^{* * *}$} & 1.069 & {$[0.000]^{* * *}$} \\
\hline Illiterate & & & & & & & 0.364 & {$[0.240]$} \\
\hline cut1 & -4.053 & {$[0.000]^{* * *}$} & -4.443 & {$[0.000]^{* * *}$} & -4.889 & {$[0.000]^{* * *}$} & -4.881 & {$[0.000]^{* * *}$} \\
\hline cut2 & -2.358 & {$[0.000]^{* * *}$} & -2.744 & {$[0.000]^{* * *}$} & -3.213 & {$[0.000]^{* * *}$} & -3.205 & {$[0.000]^{* * *}$} \\
\hline cut3 & 0.615 & {$[0.000]^{* * *}$} & 0.242 & {$[0.167]$} & -0.145 & {$[0.566]$} & -0.135 & [0.591] \\
\hline No. of observations & 5684 & & & & 5207 & & 5207 & \\
\hline Pseudo R2 & 0.00105 & & 0.223 & & 0.0302 & & 0.0303 & \\
\hline chi2 & 11.91 & & & & 268.4 & & 268.6 & \\
\hline
\end{tabular}

p-values in brackets: ${ }^{*} p<0.10,{ }^{* *} p<0.05,{ }^{* * *} p<0.01$.

Table A.20

Logit regression about the trends of relational goods and subjective well-being in Denmark.

\begin{tabular}{|c|c|c|c|c|c|c|c|c|}
\hline \multicolumn{9}{|l|}{ Denmark } \\
\hline w2 & 1.551 & {$[0.000]^{* * *}$} & 1.572 & {$[0.000]^{* * *}$} & 1.573 & {$[0.000]^{* * *}$} & 1.572 & {$[0.000]^{* * *}$} \\
\hline w4 & 1.703 & {$[0.000]^{* * *}$} & 1.735 & {$[0.000]^{* * *}$} & 1.772 & {$[0.000]^{* * *}$} & 1.774 & {$[0.000]^{* * *}$} \\
\hline Age & & & 0.0341 & {$[0.004]^{* * *}$} & 0.0253 & {$[0.094]^{*}$} & 0.0250 & {$[0.098]^{*}$} \\
\hline Age2 & & & -0.000470 & {$[0.000]^{* * *}$} & -0.000378 & {$[0.010]^{* * *}$} & -0.000375 & {$[0.010]^{* *}$} \\
\hline Male & & & 0.0595 & [0.439] & 0.0930 & {$[0.254]$} & 0.0906 & {$[0.267]$} \\
\hline No. of children & & & & & -0.101 & {$[0.010]^{* *}$} & -0.101 & {$[0.010]^{* *}$} \\
\hline Single & & & & & -0.264 & {$[0.065]^{*}$} & -0.261 & {$[0.069]^{*}$} \\
\hline Married & & & & & 0.109 & {$[0.300]$} & 0.109 & [0.298] \\
\hline Illiterate & & & & & & & -0.921 & [0.459] \\
\hline _cons & -1.481 & {$[0.000]^{* * *}$} & -1.979 & {$[0.000]^{* * *}$} & -1.657 & {$[0.000]^{* * *}$} & -1.647 & {$[0.000]^{* * *}$} \\
\hline No. of observations & 3235 & & 3235 & & 2812 & & 2812 & \\
\hline Pseudo R2 & 0.0935 & & 0.103 & & 0.0929 & & 0.0931 & \\
\hline chi2 & 352.4 & & 370.9 & & 270.6 & & 271.0 & \\
\hline Olson's groups & Mean values & & Demographic & controls & Familiar status & & Education & \\
\hline w2 & 0.389 & {$[0.000]^{* * *}$} & 0.381 & {$[0.000]^{* * *}$} & 0.566 & {$[0.000]^{* * *}$} & 0.564 & {$[0.000]^{* * *}$} \\
\hline w4 & 0.455 & {$[0.000]^{* * *}$} & 0.446 & {$[0.000]^{* * *}$} & 0.708 & {$[0.000]^{* * *}$} & 0.712 & {$[0.000]^{* * *}$} \\
\hline Age & & & 0.184 & {$[0.000]^{* * *}$} & 0.174 & {$[0.000]^{* * *}$} & 0.173 & {$[0.000]^{* * *}$} \\
\hline $\mathrm{aBe} 2$ & & & -0.00222 & {$[0.000]^{* * *}$} & -0.00211 & {$[0.000]^{* * *}$} & -0.00211 & {$[0.000]^{* * *}$} \\
\hline Male & & & 0.548 & {$[0.000]^{* * *}$} & 0.520 & {$[0.000]^{* * *}$} & 0.515 & {$[0.000]^{* * *}$} \\
\hline No. of children & & & & & -0.0955 & {$[0.018]^{* *}$} & -0.0959 & {$[0.017]^{* *}$} \\
\hline Single & & & & & -0.619 & {$[0.000]^{* * *}$} & -0.610 & {$[0.000]^{* * *}$} \\
\hline Married & & & & & -0.0213 & {$[0.849]$} & 0.0206 & {$[0.854]$} \\
\hline Illiterate & & & & & & & & \\
\hline _cons & -0.0033 & ; [0.954] & -3.384 & {$[0.000]^{* * *}$} & -3.052 & {$[0.000]^{* * *}$} & 3.036 & {$[0.000]^{* * *}$} \\
\hline No. of observations & 3235 & & 3235 & & 2812 & & 2809 & \\
\hline Pseudo R2 & 0.0075: & $\mathrm{i}$ & 0.113 & & 0.124 & & 0.123 & \\
\hline chi2 & 33.28 & & 318.2 & & 310.2 & & 309.7 & \\
\hline Other groups & Mean values & & Demographic ce & ontrols & Familiar status & & Education & \\
\hline W2 & 0.455 & {$[0.000]^{* * *}$} & 0.434 & {$[0.000]^{* * *}$} & 0.680 & {$[0.000]^{* * *}$} & 0.683 & {$[0.000]^{* * *}$} \\
\hline w4 & 0.649 & {$[0.000]^{* * *}$} & 0.618 & {$[0.000]^{* * *}$} & 0.837 & {$[0.000]^{* * *}$} & 0.833 & {$[0.000]^{* * *}$} \\
\hline Age & & & 0.0187 & {$[0.168]$} & 0.0420 & {$[0.016]^{* *}$} & 0.0432 & {$[0.013]^{* *}$} \\
\hline Age2 & & & -0.000150 & [0.273] & -0.000362 & {$[0.029]^{* *}$} & 0.000373 & {$[0.024]^{* *}$} \\
\hline Male & & & 0.116 & {$[0.184]$} & 0.145 & {$[0.121]$} & 0.153 & {$[0.104]$} \\
\hline No. of children & & & & & 0.0834 & {$[0.036]^{* *}$} & 0.0836 & {$[0.036]^{* *}$} \\
\hline Single & & & & & 0.122 & [0.482] & 0.110 & {$[0.527]$} \\
\hline Married & & & & & 0.123 & {$[0.302]$} & 0.121 & [0.309] \\
\hline Illiterate & & & & & & & 2.253 & {$[0.061]^{*}$} \\
\hline cons & -1.684 & {$[0.000]^{* * *}$} & -2.211 & {$[0.000]^{* * *}$} & -3.251 & {$[0.000]^{* * *}$} & 3.286 & {$[0.000]^{* * *}$} \\
\hline No. of observations & 3235 & & 3235 & & 2812 & & 2812 & \\
\hline Pseudo R2 & 0.0117 & & 0.0134 & & 0.0232 & & 0.0244 & \\
\hline chi2 & 37.70 & & 40.64 & & 66.82 & & 70.23 & \\
\hline Trust in others & Mean values & & Demographic cont & rols & Familiar status & & Education & \\
\hline w2 & 0.201 & {$[0.024]^{* *}$} & 0.238 & {$[0.008]^{* * *}$} & 0.344 & {$[0.001]^{* * *}$} & 0.345 & {$[0.001]^{* * *}$} \\
\hline W4 & 0.579 & {$[0.000]^{* * *}$} & 0.645 & {$[0.000]^{* * *}$} & 0.723 & {$[0.000]^{* * *}$} & 0.721 & {$[0.000]^{* * *}$} \\
\hline Age & & & 0.0127 & {$[0.267]$} & 0.0222 & {$[0.147]$} & 0.0227 & {$[0.139]$} \\
\hline Age2 & & & -0.000289 & {$[0.014]^{* *}$} & -0.000346 & {$[0.019]^{* *}$} & -0.000350 & {$[0.018]^{* *}$} \\
\hline Male & & & -0.102 & {$[0.175]$} & -0.128 & [0.117] & -0.124 & {$[0.126]$} \\
\hline No. of children & & & & & -0.0561 & {$[0.134]$} & -0.0560 & {$[0.135]$} \\
\hline Single & & & & & 0.160 & {$[0.281]$} & 0.154 & [0.298] \\
\hline Married & & & & & 0.262 & {$[0.012]^{* *}$} & 0.261 & {$[0.013]^{* *}$} \\
\hline
\end{tabular}


Table A.20 (Continued)

\begin{tabular}{|c|c|c|c|c|c|c|c|c|}
\hline \multirow{2}{*}{$\begin{array}{l}\text { Trust in others } \\
\text { _cons }\end{array}$} & \multicolumn{2}{|l|}{ Mean values } & \multicolumn{2}{|c|}{ Demographic controls } & \multirow{2}{*}{$\begin{array}{c}\text { Familiar status } \\
-0.241\end{array}$} & \multicolumn{3}{|c|}{ Education } \\
\hline & 0.108 & {$[0.080]^{*}$} & 0.215 & {$[0.400]$} & & {$[0.507]$} & -0.254 & [0.485] \\
\hline No. of observations & 3037 & & 3037 & & 2659 & & 2656 & \\
\hline Pseudo R2 & 0.0101 & & 0.0246 & & 0.0301 & & 0.0298 & \\
\hline chi2 & 40.83 & & 95.20 & & 99.06 & & 97.93 & \\
\hline Unpaid putnamian work & \multicolumn{2}{|l|}{ Mean values } & \multicolumn{2}{|c|}{ Demographic controls } & Familiar status & & Education & \\
\hline w2 & 1.234 & {$[0.000]^{* * *}$} & 1.224 & {$[0.000]^{* * *}$} & 1.21 & {$[0.000]^{* * *}$} & 1.208 & {$[0.000]^{* * *}$} \\
\hline w4 & 1.637 & {$[0.000]^{* * *}$} & 1.63 & {$[0.000]^{* * *}$} & 1.599 & {$[0.000]^{* * *}$} & 1.601 & {$[0.000]^{* * *}$} \\
\hline Age & & & 0.0434 & {$[0.009]^{* * *}$} & 0.0531 & {$[0.008]^{* * *}$} & 0.0526 & {$[0.008]^{* * *}$} \\
\hline Age2 & & & -0.000524 & {$[0.002]^{* * *}$} & -0.000605 & {$[0.002]^{* * *}$} & -0.0006 & {$[0.002]^{* * *}$} \\
\hline Male & & & 0.194 & {$[0.051]^{*}$} & 0.216 & {$[0.037]^{* *}$} & 0.211 & {$[0.040]^{* *}$} \\
\hline No. of children & & & & & -0.00257 & [0.958] & -0.0028 & [0.954] \\
\hline Single & & & & & 0.137 & [0.446] & 0.142 & {$[0.429]$} \\
\hline Married & & & & & 0.0389 & [0.773] & 0.0396 & {$[0.770]$} \\
\hline \multicolumn{9}{|l|}{ Illiterate } \\
\hline _cons & -2.714 & {$[0.000]^{* * *}$} & -3.549 & {$[0.000]^{* * *}$} & -3.84 & {$[0.000]^{* * *}$} & -3.823 & {$[0.000]^{* * *}$} \\
\hline No. of observations & 3235 & & 3235 & & 2812 & & 2809 & \\
\hline Pseudo R2 & 0.059 & & 0.0664 & & 0.0594 & & 0.0596 & \\
\hline chi2 & 122.7 & & 130.6 & & 102.2 & & 102.6 & \\
\hline Happiness & \multicolumn{2}{|l|}{ Mean values } & \multicolumn{2}{|c|}{ Demographic controls } & Familiar status & & Education & \\
\hline w2 & 0.416 & {$[0.000]^{* * *}$} & 0.433 & {$[0.000]^{* * *}$} & 0.511 & {$[0.000]^{* * *}$} & 0.510 & {$[0.000]^{* * *}$} \\
\hline w4 & 0.518 & {$[0.000]^{* * *}$} & 0.545 & {$[0.000]^{* * *}$} & 0.685 & {$[0.000]^{* * *}$} & 0.687 & {$[0.000]^{* * *}$} \\
\hline Age & & & 0.0155 & {$[0.159]$} & -0.0572 & {$[0.000]^{* * *}$} & -0.0579 & {$[0.000]^{* * *}$} \\
\hline Age2 & & & -0.000260 & {$[0.024]^{* *}$} & 0.000391 & {$[0.007]^{* * *}$} & 0.000396 & {$[0.007]^{* * *}$} \\
\hline Male & & & -0.0569 & [0.421] & 0.00439 & [0.954] & 0.00166 & [0.983] \\
\hline No. of children & & & & & -0.00201 & {$[0.956]$} & -0.00203 & {$[0.955]$} \\
\hline Single & & & & & -0.291 & {$[0.040]^{* *}$} & -0.287 & {$[0.043]^{* *}$} \\
\hline Married & & & & & 0.781 & {$[0.000]^{* * *}$} & 0.783 & {$[0.000]^{* * *}$} \\
\hline Illiterate & & & & & & & -1.427 & {$[0.448]$} \\
\hline cut1 & -4.958 & {$[0.000]^{* * *}$} & -4.890 & {$[0.000]^{* * *}$} & -6.052 & {$[0.000]^{* * *}$} & -6.070 & {$[0.000]^{* * *}$} \\
\hline cut2 & -2.671 & {$[0.000]^{* * *}$} & -2.600 & {$[0.000]^{* * *}$} & 3.835 & {$[0.000]^{* * *}$} & -3.852 & {$[0.000]^{* * *}$} \\
\hline cut3 & 0.745 & {$[0.000]^{* * *}$} & 0.837 & {$[0.000]^{* * *}$} & -0.440 & {$[0.203]$} & -0.455 & {$[0.189]$} \\
\hline No. of observations & 3189 & & & & 2775 & & 2775 & \\
\hline Pseudo R2 & 0.00765 & & 0.433 & & 0.0321 & & 0.0323 & \\
\hline chi2 & 42.79 & & & & 147.8 & & 148.2 & \\
\hline
\end{tabular}

p-values in brackets: ${ }^{*} p<0.10,{ }^{* *} p<0.05,{ }^{* * *} p<0.01$.

Table A.21

Logit regression about the trends of relational goods and subjective well-being in Norway.

\begin{tabular}{|c|c|c|c|c|c|c|c|c|}
\hline \multicolumn{8}{|l|}{ Norway } & Education \\
\hline w2 & 1.168 & {$[0.000]^{* * *}$} & 1.162 & {$[0.000]^{* * *}$} & 1.138 & {$[0.000]^{* * *}$} & 1.138 & {$[0.000]^{* * *}$} \\
\hline Age & & & 0.0423 & {$[0.008]^{* * *}$} & 0.0303 & {$[0.148]$} & 0.0303 & {$[0.148]$} \\
\hline Age2 & & & -0.000479 & {$[0.005]^{* * *}$} & -0.000367 & {$[0.083]^{*}$} & -0.000367 & {$[0.083]^{*}$} \\
\hline Male & & & -0.119 & [0.179] & -0.0696 & {$[0.460]$} & -0.0696 & {$[0.460]$} \\
\hline No. of children & & & & & 0.104 & {$[0.013]^{* *}$} & 0.104 & {$[0.013]^{* *}$} \\
\hline Single & & & & & 0.593 & {$[0.002]^{* * *}$} & 0.593 & {$[0.002]^{* * *}$} \\
\hline Married & & & & & 0.186 & {$[0.157]$} & 0.186 & {$[0.157]$} \\
\hline \multicolumn{9}{|l|}{ Illiterate } \\
\hline _cons & -0.955 & {$[0.000]^{* * *}$} & -1.695 & {$[0.000]^{* * *}$} & -1.812 & {$[0.000]^{* * *}$} & -1.812 & {$[0.000]^{* * *}$} \\
\hline No. of observations & 2290 & & 2290 & & 1997 & & 1997 & \\
\hline Pseudo R2 & 0.0574 & & 0.0608 & & 0.0562 & & 0.0562 & \\
\hline chi2 & 170.2 & & 176.8 & & 138.4 & & 138.4 & \\
\hline Olson's groups & \multicolumn{2}{|l|}{ Mean values } & \multicolumn{2}{|c|}{ Demographic controls } & \multicolumn{2}{|l|}{ Familiar status } & \multicolumn{2}{|l|}{ Education } \\
\hline w2 & 0.430 & {$[0.000]^{* * *}$} & 0.434 & {$[0.000]^{* * *}$} & 0.611 & {$[0.000]^{* * *}$} & 0.611 & {$[0.000]^{* * *}$} \\
\hline Age & & & 0.170 & {$[0.000]^{* * *}$} & 0.158 & {$[0.000]^{* * *}$} & 0.158 & {$[0.000]^{* * *}$} \\
\hline Age2 & & & -0.00177 & {$[0.000]^{* * *}$} & -0.00163 & {$[0.000]^{* * *}$} & -0.00163 & {$[0.000]^{* * *}$} \\
\hline Male & & & 0.563 & {$[0.000]^{* * *}$} & 0.617 & {$[0.000]^{* * *}$} & 0.617 & {$[0.000]^{* * *}$} \\
\hline No. of children & & & & & -0.0184 & {$[0.656]$} & -0.0184 & {$[0.656]$} \\
\hline Single & & & & & -0.630 & {$[0.002]^{* * *}$} & 0.630 & {$[0.002]^{* * *}$} \\
\hline Married & & & & & 0.0781 & {$[0.552]$} & 0.0781 & {$[0.552]$} \\
\hline \multicolumn{9}{|l|}{ Illiterate } \\
\hline _cons & -0.270 & {$[0.000]^{* * *}$} & -4.150 & {$[0.000]^{* * *}$} & -4.016 & {$[0.000]^{* * *}$} & -4.016 & {$[0.000]^{* * *}$} \\
\hline No. of observations & 2290 & & 2290 & & 1997 & & 1997 & \\
\hline Pseudo R2 & 0.00823 & & 0.0586 & & 0.0648 & & 0.0648 & \\
\hline chi2 & 25.95 & & 162.6 & & 158.5 & & 158.5 & \\
\hline Other groups & Mean values & & Demographic cont & ols & Familiar status & & Education & \\
\hline w2 & 0.746 & {$[0.000]^{* * *}$} & 0.750 & {$[0.000]^{* * *}$} & 0.707 & {$[0.000]^{* * *}$} & 0.707 & {$[0.000]^{* * *}$} \\
\hline Age & & & 0.0254 & {$[0.140]$} & 0.0147 & {$[0.500]$} & 0.0147 & {$[0.500]$} \\
\hline Age2 & & & -0.000270 & [0.134] & -0.000164 & {$[0.454]$} & -0.000164 & {$[0.454]$} \\
\hline
\end{tabular}


Table A.21 (Continued)

\begin{tabular}{|c|c|c|c|c|c|c|c|c|}
\hline \multirow{2}{*}{$\begin{array}{l}\text { Other groups } \\
\text { Male }\end{array}$} & \multicolumn{2}{|l|}{ Mean values } & \multicolumn{2}{|c|}{ Demographic controls } & \multicolumn{2}{|l|}{ Familiar status } & \multicolumn{2}{|l|}{ Education } \\
\hline & & & 0.343 & {$[0.000]^{* * *}$} & 0.348 & {$[0.001]^{* * *}$} & 0.348 & {$[0.001]^{* * *}$} \\
\hline No. of children & & & & & 0.0368 & {$[0.400]$} & 0.0368 & {$[0.400]$} \\
\hline Single & & & & & 0.0978 & {$[0.622]$} & 0.0978 & {$[0.622]$} \\
\hline Married & & & & & 0.0518 & [0.712] & 0.0518 & [0.712] \\
\hline \multicolumn{9}{|l|}{ Illiterate } \\
\hline _cons & -1.346 & {$[0.000]^{* * *}$} & -2.052 & {$[0.000]^{* * *}$} & -1.884 & {$[0.000]^{* * *}$} & -1.884 & {$[0.000]^{* * *}$} \\
\hline No. of observations & 2290 & & 2290 & & 1997 & & 1997 & \\
\hline Pseudo R2 & 0.0226 & & 0.0283 & & 0.0234 & & 0.0234 & \\
\hline chi2 & 59.62 & & 74.71 & & 55.63 & & 55.63 & \\
\hline Trust in others & \multicolumn{2}{|l|}{ Mean values } & \multicolumn{2}{|c|}{ Demographic controls } & \multicolumn{2}{|l|}{ Familiar status } & \multicolumn{2}{|l|}{ Education } \\
\hline w2 & 0.180 & {$[0.047]^{* *}$} & 0.181 & {$[0.049]^{* *}$} & 0.190 & {$[0.068]^{*}$} & 0.189 & {$[0.069]^{*}$} \\
\hline w3 & 0.191 & {$[0.037]^{* *}$} & 0.173 & {$[0.062]^{*}$} & 0.193 & {$[0.067]^{*}$} & 0.217 & {$[0.040]^{* *}$} \\
\hline Age & & & 0.0449 & {$[0.001]^{* * *}$} & 0.0368 & {$[0.032]^{* *}$} & 0.0354 & {$[0.040]^{* *}$} \\
\hline Age2 & & & -0.000670 & {$[0.000]^{* * *}$} & -0.000588 & {$[0.001]^{* * *}$} & -0.000569 & {$[0.001]^{* * *}$} \\
\hline Male & & & 0.117 & {$[0.117]$} & 0.127 & [0.109] & 0.127 & [0.109] \\
\hline No. of children & & & & & 0.0325 & {$[0.358]$} & -0.0334 & {$[0.346]$} \\
\hline Single & & & & & -0.177 & {$[0.228]$} & -0.172 & {$[0.243]$} \\
\hline Married & & & & & 0.125 & [0.219] & 0.129 & [0.209] \\
\hline Illiterate & & & & & & & -1.400 & {$[0.009]^{* * *}$} \\
\hline _cons & 0.441 & {$[0.000]^{* * *}$} & -0.0966 & {$[0.748]$} & 0.0752 & [0.847] & 0.0936 & {$[0.811]$} \\
\hline No. of observations & 3232 & & 3232 & & 2968 & & 2968 & \\
\hline Pseudo R2 & 0.00128 & & 0.0233 & & 0.0240 & & 0.0260 & \\
\hline chi2 & 5.448 & & 96.37 & & 91.16 & & 97.06 & \\
\hline Unpaid putnamian work & \multicolumn{2}{|l|}{ Mean values } & \multicolumn{2}{|c|}{ Demographic controls } & \multicolumn{2}{|l|}{ Familiar status } & \multicolumn{2}{|l|}{ Education } \\
\hline w2 & 0.823 & {$[0.000]^{* * *}$} & 0.813 & {$[0.000]^{* * *}$} & 0.938 & {$[0.000]^{* * *}$} & 0.938 & {$[0.000]^{* * *}$} \\
\hline Age & & & 0.0926 & {$[0.000]^{* * *}$} & 0.0729 & {$[0.005]^{* * *}$} & 0.0729 & {$[0.005]^{* * *}$} \\
\hline Age2 & & & -0.000953 & {$[0.000]^{* * *}$} & -0.000781 & {$[0.002]^{* * *}$} & -0.000781 & {$[0.002]^{* * *}$} \\
\hline Male & & & 0.0196 & {$[0.852]$} & 0.114 & {$[0.308]$} & 0.114 & {$[0.308]$} \\
\hline No. of children & & & & & 0.18 & {$[0.000]^{* * *}$} & 0.18 & {$[0.000]^{* * *}$} \\
\hline Single & & & & & 0.744 & {$[0.002]^{* * *}$} & 0.744 & {$[0.002]^{* * *}$} \\
\hline Married & & & & & 0.48 & {$[0.005]^{* * *}$} & 0.48 & {$[0.005]^{* * *}$} \\
\hline \multicolumn{9}{|l|}{ Illiterate } \\
\hline _cons & -1.84 & {$[0.000]^{* * *}$} & -3.831 & {$[0.000]^{* * *}$} & -4.269 & {$[0.000]^{* * *}$} & -4.269 & {$[0.000]^{* * *}$} \\
\hline No. of observations & 2290 & & 2290 & & 1997 & & 1997 & \\
\hline Pseudo R2 & 0.0252 & & 0.0348 & & 0.0438 & & 0.0438 & \\
\hline chi2 & 55.55 & & 72.37 & & 78.11 & & 78.11 & \\
\hline Happiness & Mean values & & Demographic co & ntrols & Familiar status & & Education & \\
\hline w2 & 0.0877 & {$[0.318]$} & 0.0876 & {$[0.321]$} & 0.0916 & {$[0.364]$} & 0.0912 & {$[0.368]$} \\
\hline W3 & 0.125 & [0.159] & 0.114 & {$[0.200]$} & 0.184 & {$[0.072]^{*}$} & 0.211 & {$[0.040]^{* *}$} \\
\hline Age & & & -0.00541 & {$[0.671]$} & -0.0760 & {$[0.000]^{* * *}$} & -0.0782 & {$[0.000]^{* * *}$} \\
\hline Age2 & & & -0.0000372 & {$[0.787]$} & 0.000622 & {$[0.000]^{* * *}$} & 0.000650 & {$[0.000]^{* * *}$} \\
\hline Male & & & -0.177 & {$[0.013]^{* *}$} & -0.196 & {$[0.010]^{* *}$} & -0.196 & {$[0.010]^{* *}$} \\
\hline No. of children & & & & & -0.0686 & {$[0.049]^{* *}$} & -0.0687 & {$[0.050]^{* *}$} \\
\hline Single & & & & & -0.371 & {$[0.010]^{* *}$} & -0.365 & {$[0.012]^{* *}$} \\
\hline Married & & & & & 0.844 & {$[0.000]^{* * *}$} & 0.847 & {$[0.000]^{* * *}$} \\
\hline Illiterate & & & & & & & -1.783 & {$[0.002]^{* * *}$} \\
\hline cut1 & -4.834 & {$[0.000]^{* * *}$} & -5.260 & {$[0.000]^{* * *}$} & -6.674 & {$[0.000]^{* * *}$} & -6.727 & {$[0.000]^{* * *}$} \\
\hline cut2 & -2.639 & {$[0.000]^{* * *}$} & -3.062 & {$[0.000]^{* * *}$} & -4.451 & {$[0.000]^{* * *}$} & -4.496 & {$[0.000]^{* * *}$} \\
\hline cut3 & 0.960 & {$[0.000]^{* * *}$} & 0.555 & {$[0.040]^{* *}$} & 0.728 & {$[0.040]^{* *}$} & -0.758 & {$[0.032]^{* *}$} \\
\hline No. of observations & 3402 & & & & 3109 & & 3109 & \\
\hline Pseudo R2 & 0.000365 & & 0.0876 & & 0.0264 & & 0.0288 & \\
\hline chi2 & 2.058 & & & & 133.8 & & 148.8 & \\
\hline
\end{tabular}

$p$-values in brackets: ${ }^{*} p<0.10,{ }^{* *} p<0.05,{ }^{* * *} p<0.01$.

Table A.22

Logit regression about the trends of relational goods and subjective well-being in Sweden

\begin{tabular}{|c|c|c|c|c|c|c|c|c|}
\hline \multirow{2}{*}{$\begin{array}{l}\text { Sweden } \\
\text { Putnam's groups } \\
\text { w2 }\end{array}$} & \multicolumn{2}{|l|}{ Mean values } & \multicolumn{2}{|c|}{ Demographic controls } & \multicolumn{2}{|l|}{ Familiar status } & \multicolumn{2}{|l|}{ Education } \\
\hline & 1218 & {$[0.000]^{* * *}$} & 1.219 & {$[0.000]^{* * *}$} & 1.192 & {$[0.000]^{* * *}$} & 1.193 & {$[0.000]^{* * *}$} \\
\hline w4 & 3.039 & {$[0.000]^{* * *}$} & 3.036 & {$[0.000]^{* * *}$} & 3.032 & {$[0.000]^{* * *}$} & 3.038 & {$[0.000]^{* * *}$} \\
\hline Age & & & 0.0191 & {$[0.236]$} & 0.0140 & {$[0.493]$} & 0.0134 & {$[0.510]$} \\
\hline Age2 & & & -0.000239 & {$[0.176]$} & -0.000201 & {$[0.344]$} & -0.000194 & {$[0.360]$} \\
\hline Male & & & -0.189 & {$[0.030]^{* *}$} & -0.178 & {$[0.053]^{*}$} & -0.177 & {$[0.054]^{*}$} \\
\hline No. of children & & & & & 0.00808 & {$[0.863]$} & 0.00795 & {$[0.865]$} \\
\hline Single & & & & & 0.313 & {$[0.066]^{*}$} & 0.312 & {$[0.067]^{*}$} \\
\hline Married & & & & & 0.196 & {$[0.082]^{*}$} & 0.199 & {$[0.077]^{*}$} \\
\hline Illiterate & & & & & & & -0.856 & {$[0.475]$} \\
\hline _coris & -0.982 & {$[0.000]^{* * *}$} & -1.197 & {$[0.001]^{* * *}$} & 1.220 & {$[0.007]^{* * *}$} & -1.210 & {$[0.007]^{* * *}$} \\
\hline No. of observations & 3016 & & 2962 & & 2700 & & 2700 & \\
\hline Pseudo R2 & 0.203 & & 0.209 & & 0.202 & & 0.202 & \\
\hline chi2 & 615.0 & & 630.0 & & 552.2 & & 552.0 & \\
\hline
\end{tabular}


Table A.22 (Continued)

\begin{tabular}{|c|c|c|c|c|c|c|c|c|c|}
\hline \multirow{2}{*}{$\begin{array}{l}\text { Olson's groups } \\
\text { w2 }\end{array}$} & \multicolumn{2}{|l|}{ Mean values } & \multicolumn{3}{|c|}{ Demographic controls } & \multicolumn{2}{|l|}{ Familiar status } & \multicolumn{2}{|l|}{ Education } \\
\hline & 0.555 & {$[0.000]^{* * *}$} & 0.589 & {$[0.000]^{* * *}$} & & 0.730 & $0.000]^{* * *}$ & 0.732 & {$[0.000]^{* * *}$} \\
\hline w4 & 0.887 & {$[0.000]^{* * *}$} & 0.935 & {$[0.000]^{* * *}$} & & 1.074 & $0.000]^{* * *}$ & 1.081 & {$[0.000]^{* * *}$} \\
\hline Age & & & 0.210 & {$[0.000]^{* * *}$} & & 0.201 & $0.000]^{* * *}$ & 0.200 & {$[0.000]^{* * *}$} \\
\hline Age2 & & & -0.00239 & {$[0.000]^{* * *}$} & & 0.00230 & $0.000]^{* * *}$ & -0.00229 & {$[0.000]^{* * *}$} \\
\hline Male & & & 0.261 & {$[0.001]^{* * *}$} & & 0.251 & $0.003]^{* * *}$ & 0.254 & {$[0.003]^{* * *}$} \\
\hline No. of children & & & & & & -0.0434 & $0.330]$ & -0.0433 & {$[0.331]$} \\
\hline Single & & & & & & -0.557 & $0.000]^{* * *}$ & -0.559 & {$[0.000]^{* * *}$} \\
\hline Married & & & & & & 0.0607 & $0.566]$ & -0.0550 & {$[0.603]$} \\
\hline Illiterate & & & & & & & & -1.396 & {$[0.323]$} \\
\hline _coris & -0.0377 & {$[0.560]$} & -4.162 & {$[0.000]^{* * *}$} & & 3.896 & $0.000]^{* * *}$ & -3.876 & {$[0.000]^{* * *}$} \\
\hline No. of observations & 3016 & & 2962 & & & 2700 & & 2700 & \\
\hline Pseudo R2 & 0.0231 & & 0.0865 & & & 0.0868 & & 0.0872 & \\
\hline chi2 & 91.50 & & 233.6 & & & 209.9 & & 210.4 & \\
\hline Other groups & Mean values & & Demographic c & trols & & Familiar status & & Education & \\
\hline W2 & 0.898 & {$[0.000]^{* * *}$} & 0.926 & {$[0.000]^{* * *}$} & & 0.973 & $0.000]^{* * *}$ & 0.974 & {$[0.000]^{* * *}$} \\
\hline w4 & 1.022 & {$[0.000]^{* * *}$} & 1.042 & {$[0.000]^{* * *}$} & & 1.105 & $0.000]^{* * *}$ & 1.110 & {$[0.000]^{* * *}$} \\
\hline Age & & & 0.0307 & {$[0.043]^{* *}$} & & 0.00777 & $0.673]$ & 0.00697 & {$[0.706]$} \\
\hline Age2 & & & -0.000191 & {$[0.240]$} & & 0.0000116 & $0.951]$ & 0.0000206 & {$[0.914]$} \\
\hline Male & & & 0.0769 & {$[0.348]$} & & 0.130 & $0.126]$ & 0.131 & {$[0.123]$} \\
\hline No. of children & & & & & & 0.0581 & $0.161]$ & 0.0579 & {$[0.163]$} \\
\hline Single & & & & & & 0.260 & $0.087]^{*}$ & -0.262 & {$[0.086]^{*}$} \\
\hline Married & & & & & & 0.129 & $0.203]$ & 0.133 & [0.191] \\
\hline Illiterate & & & & & & & & -0.899 & [0.439] \\
\hline _cons & -1.514 & {$[0.000]^{* * *}$} & -2.517 & {$[0.000]^{* * *}$} & & 2.137 & $0.000]^{* * *}$ & -2.124 & {$[0.000]^{* * *}$} \\
\hline No. of observations & 3016 & & 2962 & & & 2700 & & 2700 & \\
\hline Pseudo R2 & 0.0303 & & 0.0384 & & & 0.0354 & & 0.0356 & \\
\hline chi2 & 102.1 & & 123.9 & & & 114.9 & & 115.6 & \\
\hline Trust in others & Mean values & & Demographic & ntrols & & Familiar status & & Education & \\
\hline w2 & 0.397 & {$[0.000]^{* * *}$} & 0.375 & {$[0.000]^{* * *}$} & & 0.481 & {$[0.000]^{* * *}$} & 0.481 & {$[0.000]^{* * *}$} \\
\hline w3 & 0.120 & {$[0.204]$} & 0.114 & {$[0.234]$} & & 0.234 & {$[0.028]^{* *}$} & 0.235 & {$[0.028]^{* *}$} \\
\hline W4 & 0.407 & {$[0.000]^{* * *}$} & 0.393 & {$[0.000]^{* * *}$} & & 0.527 & {$[0.000]^{* * *}$} & 0.528 & {$[0.000]^{* * *}$} \\
\hline Age & & & 0.0352 & {$[0.005]^{* * *}$} & & 0.0245 & {$[0.112]$} & 0.0244 & {$[0.113]$} \\
\hline Age2 & & & -0.000479 & {$[0.000]^{* * *}$} & & -0.000383 & {$[0.015]^{* *}$} & -0.000382 & {$[0.015]^{* *}$} \\
\hline Male & & & 0.0574 & {$[0.402]$} & & 0.0582 & {$[0.417]$} & 0.0585 & {$[0.415]$} \\
\hline No. of children & & & & & & -0.0328 & {$[0.373]$} & -0.0328 & {$[0.373]$} \\
\hline Single & & & & & & -0.0952 & {$[0.440]$} & -0.0952 & {$[0.440]$} \\
\hline Married & & & & & & 0.347 & {$[0.000]^{* * *}$} & 0.348 & {$[0.000]^{* * *}$} \\
\hline Illiterate & & & & & & & & -0.178 & [0.819] \\
\hline cons & 0.271 & {$[0.000]^{* * *}$} & 0.240 & [0.389] & & -0.205 & {$[0.554]$} & -0.203 & {$[0.557]$} \\
\hline No. of observations & 3751 & & 3700 & & & 3452 & & 3452 & \\
\hline Pseudo R2 & 0.00541 & & 0.0121 & & & 0.0172 & & 0.0172 & \\
\hline chi2 & 26.81 & & 58.33 & & & 75.96 & & 76.09 & \\
\hline Unpaid putnamian work & Mean values & & Demog & phic controls & & Familiar sta & tatus & Education & \\
\hline w2 & 0.545 & {$[0.000]^{* * *}$} & 0.5 & & $0.000]^{* * *}$ & 0.434 & {$[0.000]^{* * *}$} & 0.434 & {$[0.000]^{* * *}$} \\
\hline w4 & 1.466 & {$[0.000]^{* * *}$} & 1.4 & {$[0$} & $0.000]^{* * *}$ & 1.406 & {$[0.000]^{* * *}$} & 1.407 & {$[0.000]^{* * *}$} \\
\hline Age & & & 0.0 & {$[0$} & $0.072]^{*}$ & 0.0199 & [0.288] & 0.0199 & {$[0.290]$} \\
\hline Age2 & & & -0.0 & 359 & $0.037]^{* *}$ & -0.000297 & 97 [0.129] & -0.000296 & {$[0.130]$} \\
\hline Male & & & 0.2 & & $0.001]^{* * *}$ & 0.291 & {$[0.001]^{* * *}$} & 0.292 & {$[0.001]^{* * *}$} \\
\hline No. of children & & & & & & 0.0466 & {$[0.279]$} & 0.0466 & {$[0.279]$} \\
\hline Single & & & & & & 0.19 & {$[0.210]$} & 0.19 & {$[0.210]$} \\
\hline Married & & & & & & 0.181 & {$[0.086]^{*}$} & 0.181 & {$[0.085]^{*}$} \\
\hline Illiterate & & & & & & & & -0.0574 & [0.959] \\
\hline _cons & -1.529 & {$[0.000]^{* * *}$} & -2.1 & & $0.000]^{* * *}$ & -2.034 & {$[0.000]^{* * *}$} & 2.033 & {$[0.000]^{* * *}$} \\
\hline No. of observations & 3016 & & 2962 & & & 2700 & & 2700 & \\
\hline Pseudo R2 & 0.0604 & & 0.0 & & & 0.0607 & & 0.0607 & \\
\hline chi2 & 209.7 & & 228.2 & & & 196 & & 196 & \\
\hline Happiness & Mean values & & Demographic c & trols & & Familiar status & & Education & \\
\hline w2 & 0.463 & {$[0.000]^{* * *}$} & 0.461 & {$[0.000]^{* * *}$} & & 0.633 & {$[0.000]^{* * *}$} & 0.633 & {$[0.000]^{* * *}$} \\
\hline w3 & 0.393 & {$[0.000]^{* * *}$} & 0.393 & {$[0.000]^{* * *}$} & & 0.607 & {$[0.000]^{* * *}$} & 0.608 & {$[0.000]^{* * *}$} \\
\hline W4 & 0.236 & {$[0.010]^{* * *}$} & 0.229 & {$[0.013]^{* *}$} & & 0.464 & {$[0.000]^{* * *}$} & 0.465 & {$[0.000]^{* * *}$} \\
\hline Age & & & -0.0182 & {$[0.115]$} & & -0.112 & {$[0.000]^{* * *}$} & -0.112 & {$[0.000]^{* * *}$} \\
\hline Age2 & & & 0.000125 & [0.321] & & 0.000970 & {$[0.000]^{* * *}$} & 0.000972 & {$[0.000]^{* * *}$} \\
\hline Male & & & -0.274 & {$[0.000]^{* * *}$} & & -0.298 & {$[0.000]^{* * *}$} & -0.298 & {$[0.000]^{* * *}$} \\
\hline No. of children & & & & & & -0.0324 & [0.339] & -0.0324 & {$[0.339]$} \\
\hline Single & & & & & & -0.760 & {$[0.000]^{* * *}$} & -0.760 & {$[0.000]^{* * *}$} \\
\hline Married & & & & & & 0.939 & {$[0.000]^{* * *}$} & 0.940 & {$[0.000]^{* * *}$} \\
\hline Illiterate & & & & & & & & -0.277 & {$[0.804]$} \\
\hline cut1 & -4.406 & {$[0.000]^{* * *}$} & 5.082 & {$[0.000]^{* * *}$} & & -6.948 & {$[0.000]^{* * *}$} & -6.952 & {$[0.000]^{* * *}$} \\
\hline cut2 & -2.719 & {$[0.000]^{* * *}$} & -3.404 & {$[0.000]^{* * *}$} & & -5.304 & {$[0.000]^{* * *}$} & -5.308 & {$[0.000]^{* * *}$} \\
\hline cut3 & 0.828 & {$[0.000]^{* * *}$} & 0.159 & [0.528] & & -1.596 & {$[0.000]^{* * *}$} & -1.599 & {$[0.000]^{* * *}$} \\
\hline No. of observations & 3982 & & 3928 & & & 3662 & & 3662 & \\
\hline Pseudo R2 & 0.00452 & & 0.00915 & & & 0.0463 & & 0.0463 & \\
\hline chi2 & 32.22 & & 60.20 & & & 268.4 & & 268.4 & \\
\hline
\end{tabular}

p-values in brackets: ${ }^{*} p<0.10,{ }^{* *} p<0.05,{ }^{* * *} p<0.01$. 
Table A.23

Logit regression about the trends of relational goods and subjective well-being in Finland.

\begin{tabular}{|c|c|c|c|c|c|c|c|c|}
\hline \multirow{2}{*}{$\begin{array}{l}\text { Finland } \\
\text { Putnam's groups } \\
\text { w4 }\end{array}$} & \multicolumn{2}{|l|}{ Mean values } & \multicolumn{2}{|c|}{ Demographic controls } & \multicolumn{2}{|l|}{ Familiar status } & \multicolumn{2}{|l|}{ Education } \\
\hline & 0.577 & {$[0.000]^{* * *}$} & 0.575 & {$[0.000]^{* * *}$} & 0.640 & {$[0.000]^{* * *}$} & 0.640 & {$[0.000]^{* * *}$} \\
\hline Age & & & 0.0113 & {$[0.552]$} & -0.00449 & {$[0.833]$} & -0.00449 & {$[0.833]$} \\
\hline Age2 & & & -0.0000883 & {$[0.668]$} & 0.0000581 & [0.796] & 0.0000581 & {$[0.796]$} \\
\hline Male & & & -0.138 & [0.189] & -0.140 & {$[0.197]$} & -0.140 & {$[0.197]$} \\
\hline No. of children & & & & & -0.0149 & {$[0.692]$} & -0.0149 & {$[0.692]$} \\
\hline Single & & & & & -0.153 & {$[0.390]$} & -0.153 & {$[0.390]$} \\
\hline Married & & & & & 0.232 & {$[0.076]^{*}$} & 0.232 & {$[0.076]^{*}$} \\
\hline \multicolumn{9}{|l|}{ Illiterate } \\
\hline _cons & 0.191 & {$[0.021]^{* *}$} & -0.0368 & [0.930] & 0.232 & [0.619] & 0.232 & [0.619] \\
\hline No. of observations & 1626 & & 1605 & & 1532 & & 1532 & \\
\hline Pseudo R2 & 0.0138 & & 0.0152 & & 0.0183 & & 0.0183 & \\
\hline chi2 & 29.38 & & 31.99 & & 36.47 & & 36.47 & \\
\hline Olson's groups & \multicolumn{2}{|l|}{ Mean values } & \multicolumn{2}{|c|}{ Demographic controls } & \multicolumn{2}{|l|}{ Familiar status } & \multicolumn{2}{|l|}{ Education } \\
\hline w4 & -0.243 & {$[0.019]^{* *}$} & -0.105 & {$[0.331]$} & 0.00879 & [0.939] & 0.00879 & [0.939] \\
\hline Age & & & 0.206 & {$[0.000]^{* * *}$} & 0.175 & {$[0.000]^{* * *}$} & 0.175 & {$[0.000]^{* * *}$} \\
\hline Age2 & & & 0.00228 & {$[0.000]^{* * *}$} & -0.00197 & {$[0.000]^{* * *}$} & -0.00197 & {$[0.000]^{* * *}$} \\
\hline Male & & & -0.0708 & {$[0.497]$} & -0.0829 & {$[0.445]$} & -0.0829 & {$[0.445]$} \\
\hline No. of children & & & & & -0.0481 & [0.207] & -0.0481 & {$[0.207]$} \\
\hline Single & & & & & -0.297 & {$[0.107]$} & -0.297 & [0.107] \\
\hline Married & & & & & 0.558 & {$[0.000]^{* * *}$} & 0.558 & {$[0.000]^{* * *}$} \\
\hline \multicolumn{9}{|l|}{ Illiterate } \\
\hline _cons & -0.0953 & [0.249] & -4.281 & {$[0.000]^{* * *}$} & -3.821 & {$[0.000]^{* * *}$} & -3.821 & {$[0.000]^{* * *}$} \\
\hline No. of observations & 1626 & & 1605 & & 1532 & & 1532 & \\
\hline Pseudo R2 & 0.00246 & & 0.0513 & & 0.0664 & & 0.0664 & \\
\hline chi2 & 5.479 & & 96.65 & & 124.1 & & 124.1 & \\
\hline Other groups & \multicolumn{2}{|l|}{ Mean values } & Demographic co & ntrols & Familiar status & & Education & \\
\hline w4 & -0.0754 & {$[0.524]$} & 0.0986 & {$[0.411]$} & -0.0114 & [0.928] & -0.0114 & {$[0.928]$} \\
\hline age & & & 0.0315 & [0.158] & 0.00862 & [0.725] & 0.00862 & {$[0.725]$} \\
\hline Age2 & & & -0.000129 & {$[0.581]$} & 0.0000810 & {$[0.748]$} & 0.0000810 & {$[0.748]$} \\
\hline Male & & & 0.0233 & {$[0.840]$} & -0.0370 & {$[0.754]$} & -0.0370 & {$[0.754]$} \\
\hline No. of children & & & & & 0.00475 & {$[0.901]$} & 0.00475 & {$[0.901]$} \\
\hline Single & & & & & -0.137 & {$[0.522]$} & -0.137 & [0.522] \\
\hline Married & & & & & 0.343 & {$[0.015]^{* *}$} & 0.343 & {$[0.015]^{* *}$} \\
\hline Illiterate & & & & & & & & \\
\hline _cons & -1.036 & {$[0.000]^{* * *}$} & -2.095 & {$[0.000]^{* * *}$} & -1.771 & {$[0.001]^{* * *}$} & -1.771 & {$[0.001]^{* * *}$} \\
\hline No. of observations & 1626 & & 1605 & & 1532 & & 1532 & \\
\hline Pseudo R2 & $0.00022]$ & & 0.0154 & & 0.0202 & & 0.0202 & \\
\hline chi2 & 0.407 & & 26.96 & & 33.81 & & 33.81 & \\
\hline Trust in others & Mean values & & Demographic con & trols & Familiar status & & Education & \\
\hline w3 & -0.568 & {$[0.000]^{* * *}$} & -0.594 & {$[0.000]^{* * *}$} & -0.518 & {$[0.000]^{* * *}$} & -0.518 & {$[0.000]^{* * *}$} \\
\hline w4 & -0.221 & {$[0.041]^{* *}$} & -0.237 & {$[0.031]^{* *}$} & -0.143 & {$[0.203]$} & -0.143 & {$[0.203]$} \\
\hline Age & & & -0.0312 & {$[0.031]^{* *}$} & -0.0442 & {$[0.006]^{* * *}$} & -0.0442 & {$[0.006]^{* * *}$} \\
\hline Age2 & & & 0.000293 & {$[0.061]^{*}$} & 0.000415 & {$[0.014]^{* *}$} & 0.000415 & {$[0.014]^{* *}$} \\
\hline Male & & & -0.140 & {$[0.083]^{*}$} & -0.171 & {$[0.040]^{* *}$} & -0.171 & {$[0.040]^{* *}$} \\
\hline No. of children & & & & & 0.0139 & [0.639] & 0.0139 & {$[0.639]$} \\
\hline Single & & & & & 0.191 & {$[0.144]$} & 0.191 & {$[0.144]$} \\
\hline Married & & & & & 0.367 & {$[0.000]^{* * *}$} & 0.367 & {$[0.000]^{* * *}$} \\
\hline Illiterate & & & & & & & & \\
\hline _cons & 0.520 & {$[0.000]^{* * *}$} & 1.327 & {$[0.000]^{* * *}$} & 1.341 & {$[0.000]^{* * *}$} & 1.341 & {$[0.000]^{* * *}$} \\
\hline No. of observations & 2542 & & 2520 & & 2451 & & 2451 & \\
\hline Pseudo R2 & 0.00884 & & 0.0117 & & 0.0166 & & 0.0166 & \\
\hline chi2 & 30.61 & & 39.74 & & 53.31 & & 53.31 & \\
\hline Unpaid putnamian work & Mean values & & Demographic & controls & Familiar status & & Education & \\
\hline w4 & -0.138 & {$[0.214]$} & -0.151 & [0.178] & 0.0369 & {$[0.756]$} & -0.0369 & {$[0.756]$} \\
\hline Age & & & 0.0427 & {$[0.037]^{* *}$} & 0.0167 & {$[0.462]$} & 0.0167 & {$[0.462]$} \\
\hline Age2 & & & -0.000349 & [0.108] & -0.000103 & [0.662] & -0.000103 & {$[0.662]$} \\
\hline Male & & & 0.0726 & [0.507] & 0.0656 & {$[0.560]$} & 0.0656 & {$[0.560]$} \\
\hline No. of children & & & & & 0.00492 & [0.898] & 0.00492 & {$[0.898]$} \\
\hline Single & & & & & -0.187 & {$[0.342]$} & -0.187 & {$[0.342]$} \\
\hline Married & & & & & 0.304 & {$[0.027]^{* *}$} & 0.304 & {$[0.027]^{* *}$} \\
\hline Illiterate & & & & & & & & \\
\hline _cons & -0.655 & {$[0.000]^{* * *}$} & -1.795 & {$[0.000]^{* * *}$} & -1.394 & {$[0.006]^{* * *}$} & -1.394 & {$[0.006]^{* * *}$} \\
\hline No. of observations & 1626 & & 1605 & & 1532 & & 1532 & \\
\hline Pseudo R2 & 0.00078 & & 0.0062 & & 0.0109 & & 0.0109 & \\
\hline chi2 & 1.545 & & 12.9 & & 20.54 & & 20.54 & \\
\hline
\end{tabular}


Table A.23 (Continued)

\begin{tabular}{|c|c|c|c|c|c|c|c|c|}
\hline \multirow{2}{*}{$\begin{array}{l}\text { Happiness } \\
\text { w3 }\end{array}$} & \multicolumn{2}{|l|}{ Mean values } & \multicolumn{2}{|c|}{ Demographic controls } & \multicolumn{2}{|l|}{ Familiar status } & \multicolumn{2}{|l|}{ Education } \\
\hline & 0.236 & {$[0.032]^{* *}$} & 0.188 & {$[0.091]^{*}$} & 0.418 & {$[0.000]^{* * *}$} & 0.418 & {$[0.000]^{* * *}$} \\
\hline w4 & 0.202 & {$[0.071]^{*}$} & 0.182 & [0.109] & 0.429 & {$[0.000]^{* * *}$} & 0.429 & {$[0.000]^{* * *}$} \\
\hline Age & & & -0.0695 & {$[0.000]^{* * *}$} & -0.140 & {$[0.000]^{* * *}$} & -0.140 & {$[0.000]^{* * *}$} \\
\hline Age2 & & & 0.000637 & {$[0.000]^{* * *}$} & 0.00131 & {$[0.000]^{* * *}$} & 0.00131 & {$[0.000]^{* * *}$} \\
\hline Male & & & -0.216 & {$[0.010]^{* * *}$} & -0.205 & {$[0.018]^{* *}$} & -0.205 & {$[0.018]^{* *}$} \\
\hline No. of children & & & & & -0.0460 & {$[0.160]$} & -0.0460 & {$[0.160]$} \\
\hline Single & & & & & -0.553 & {$[0.000]^{* * *}$} & -0.553 & {$[0.000]^{* * *}$} \\
\hline Married & & & & & 0.833 & {$[0.000]^{* * *}$} & 0.833 & {$[0.000]^{* * *}$} \\
\hline \multicolumn{9}{|l|}{ Illiterate } \\
\hline cut1 & -4.210 & {$[0.000]^{* * *}$} & -6.003 & {$[0.000]^{* * *}$} & -7.287 & {$[0.000]^{* * *}$} & -7.287 & {$[0.000]^{* * *}$} \\
\hline cut2 & -2.141 & {$[0.000]^{* * *}$} & -3.937 & {$[0.000]^{* * *}$} & -5.237 & {$[0.000]^{* * *}$} & -5.237 & {$[0.000]^{* * *}$} \\
\hline cut3 & 1.353 & {$[0.000]^{* * *}$} & -0.398 & {$[0.235]$} & -1.533 & {$[0.000]^{* * *}$} & -1.533 & {$[0.000]^{* * *}$} \\
\hline No. of observations & 2575 & & & & 2482 & & 2482 & \\
\hline Pseudo R2 & 0.00110 & & 0.188 & & 0.0383 & & 0.0383 & \\
\hline chi2 & 4.891 & & & & 145.1 & & 145.1 & \\
\hline
\end{tabular}

p-values in brackets: ${ }^{*} p<0.10,{ }^{* *} p<0.05,{ }^{* * *} p<0.01$.

\section{Appendix B.}

Figs. B.1-B.19
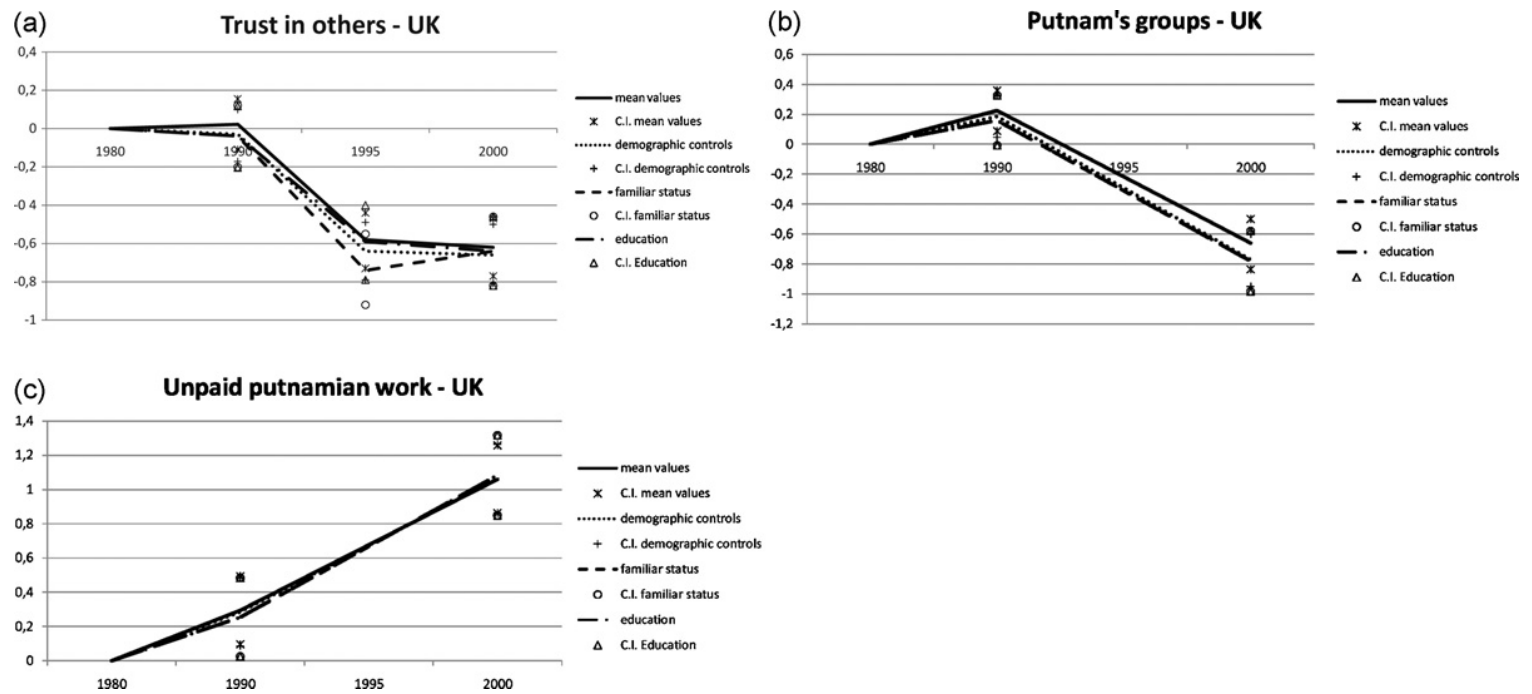

Fig. B.1. Relational social capital trends for Great Britain from 1980 to 2000. (a) trust in others; (b) membership in Putnam's groups; (c) unpaid voluntary work in putnamian groups.

(a) Olson's groups - UK

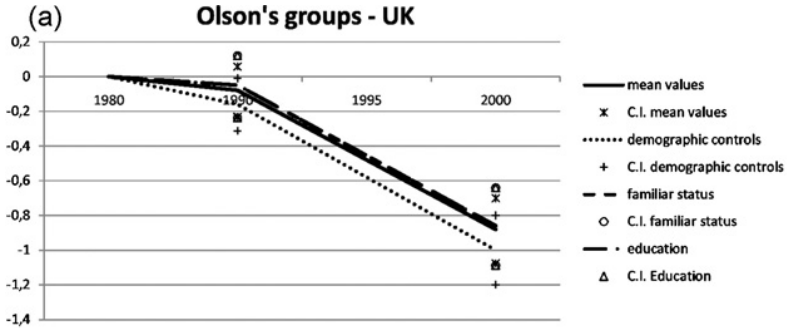

(c) Unpaid olsonian work - UK

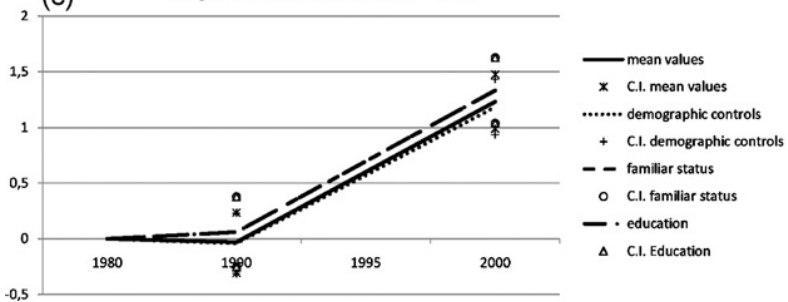

(b) Other groups - UK
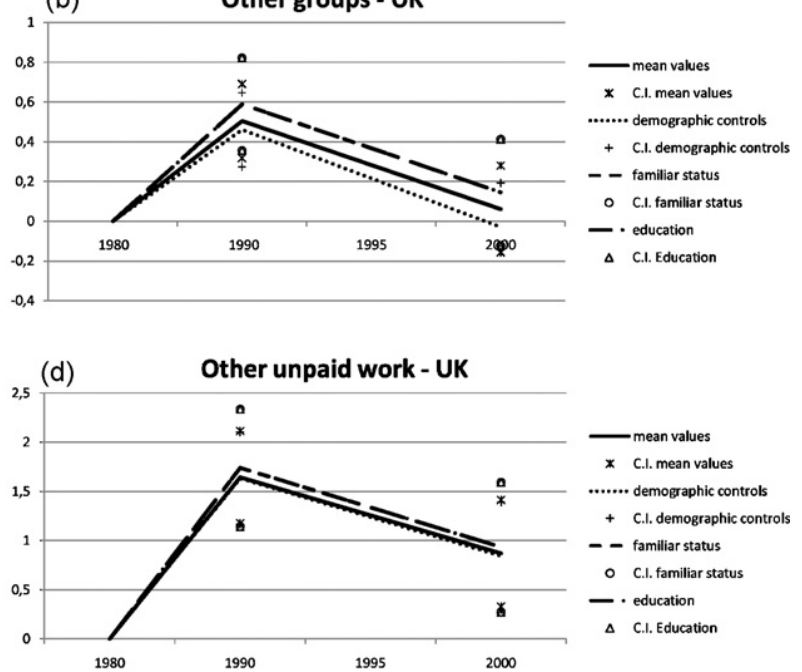

Fig. B.2. Trends about membership and unpaid voluntary work in Olson's and other groups for Great Britain from 1980 to 2000 . Membership in Olson's (a) and other groups (b); performing unpaid voluntary work in olsonian (c) and other (d) groups. 
(a)

Confidence in church - UK

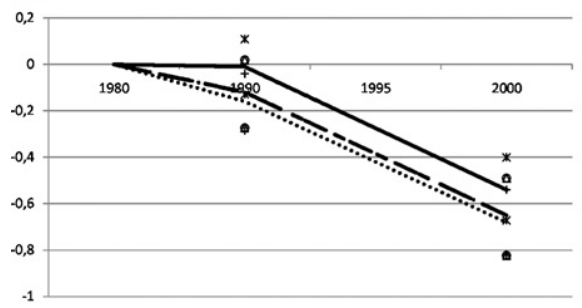

(b) Confidence in the judicial system - UK

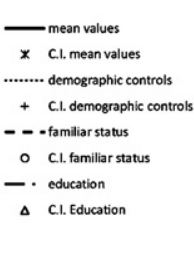

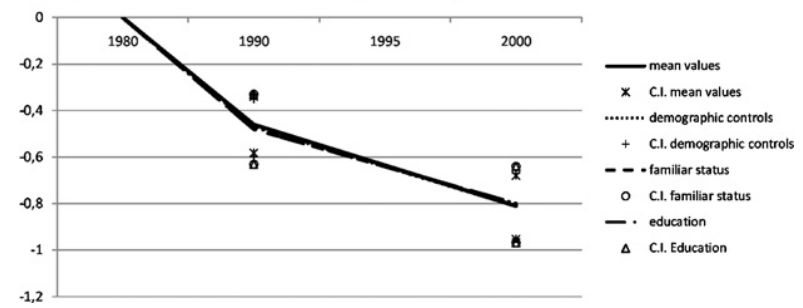

Fig. B.3. Trends about confidence in institutions for Great Britain from 1980 to 2000. (a) Confidence in religious institutions; (b) confidence in judicial system.

(a) Confidence in the parliament - UK

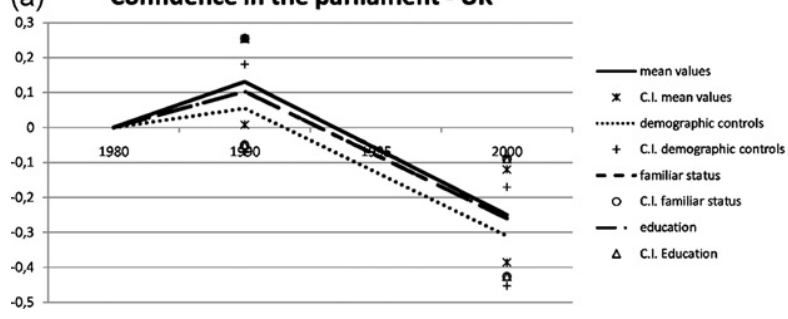

(b) Confidence in civil service - UK

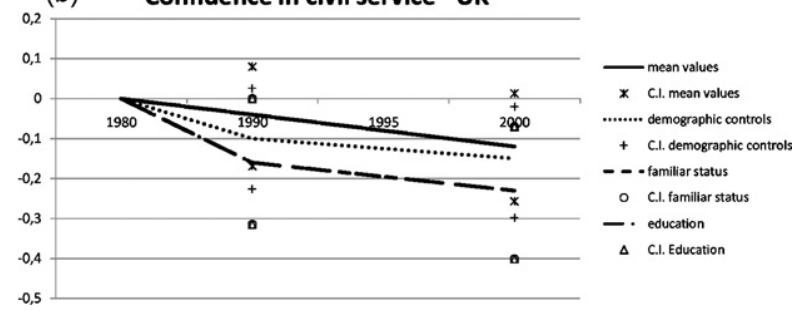

Fig. B.4. Trends about confidence in institutions for Great Britain from 1980 to 2000. (a) Confidence in parliament; (b) confidence in civil services.
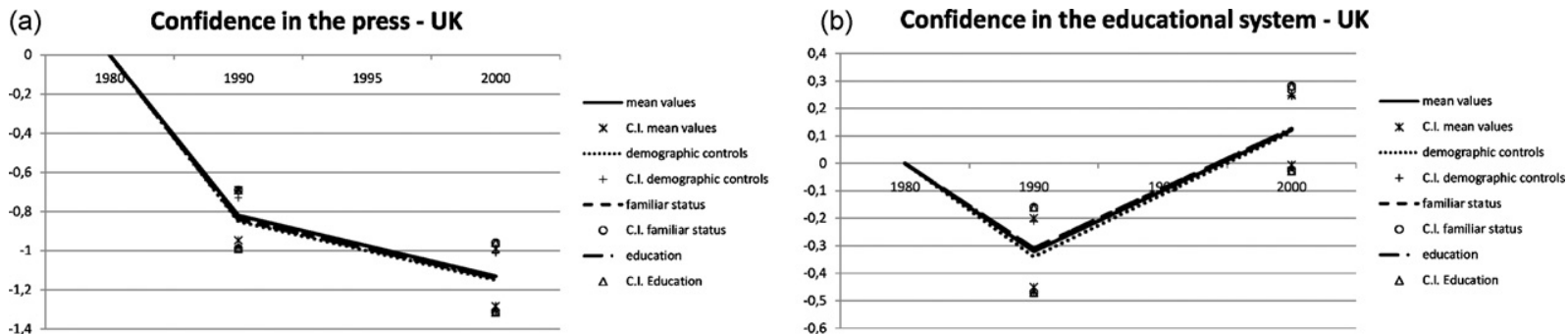

Fig. B.5. Trends about confidence in institutions for Great Britain from 1980 to 2000. (a) Confidence in press; (b) confidence in educational system.
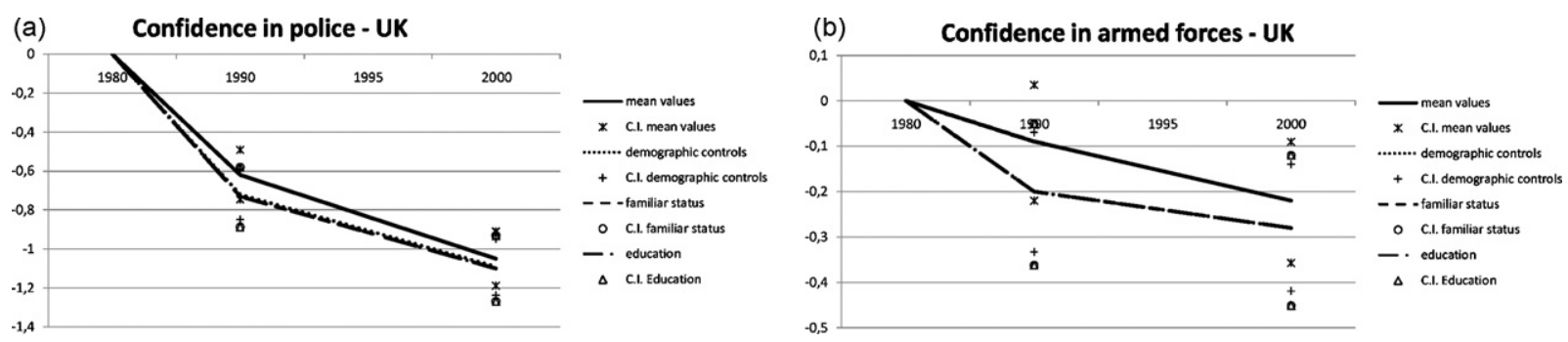

Fig. B.6. Trends about confidence in institutions for Great Britain from 1980 to 2000. (a) Confidence in police; (b) confidence in armed forces.

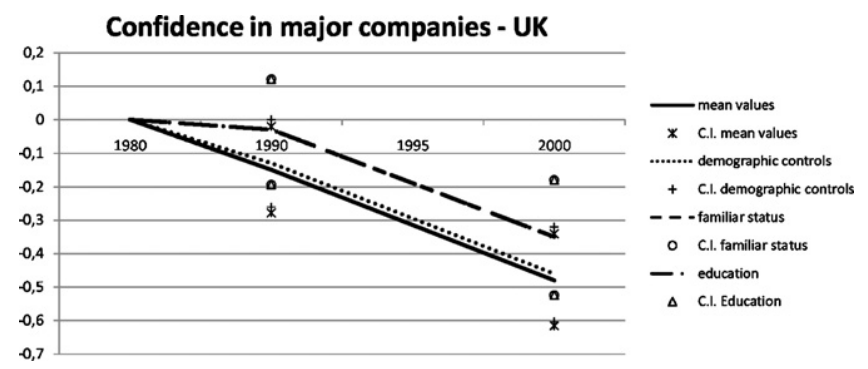

Fig. B.7. Trends about confidence in major companies for Great Britain from 1980 to 2000 . 

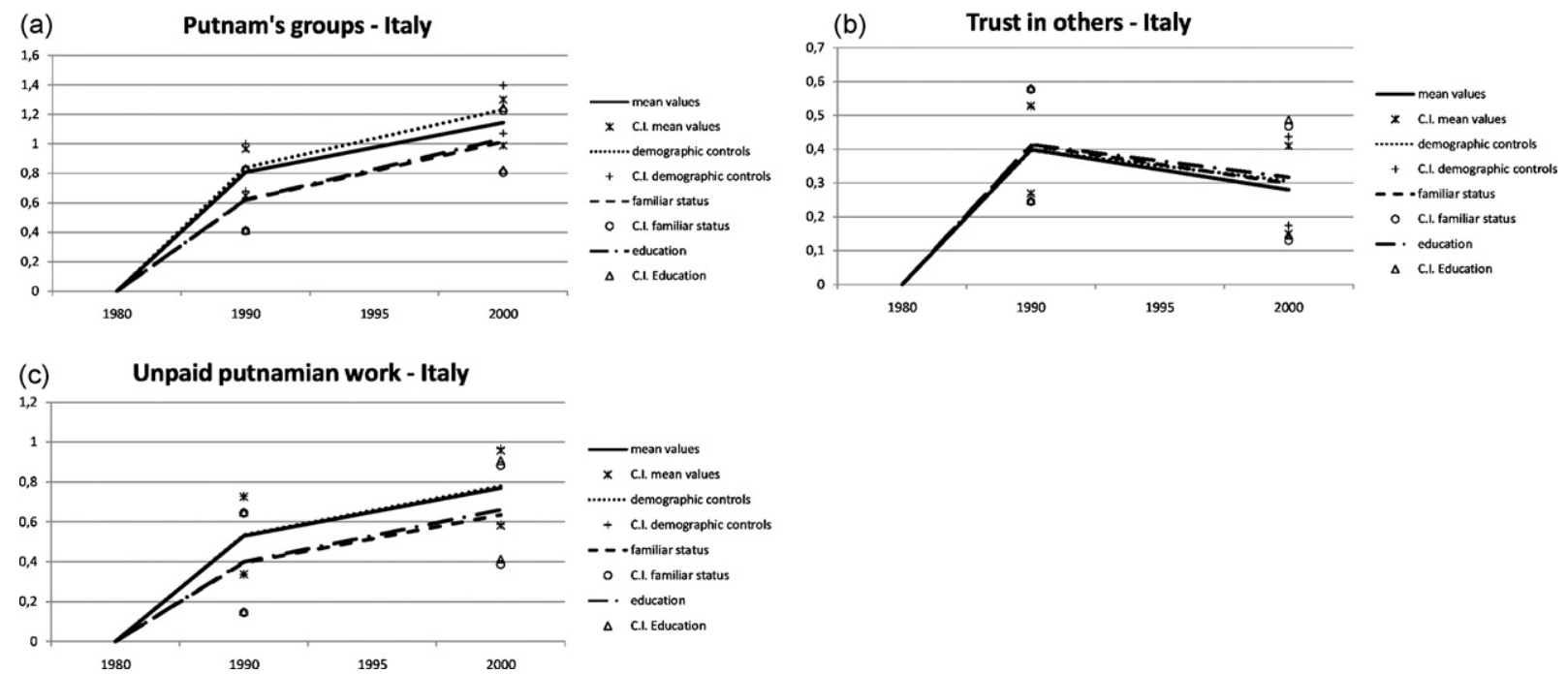

Fig. B.8. Trends about membership in Putnam's groups (a), trust in others (b) and unpaid putnamian voluntary work (c) for Italy from 1980 to 2000.
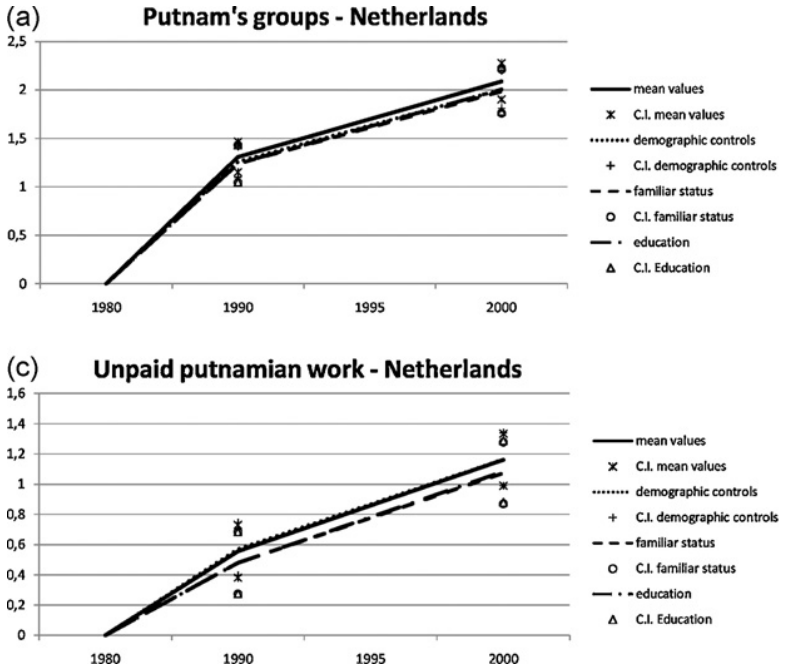

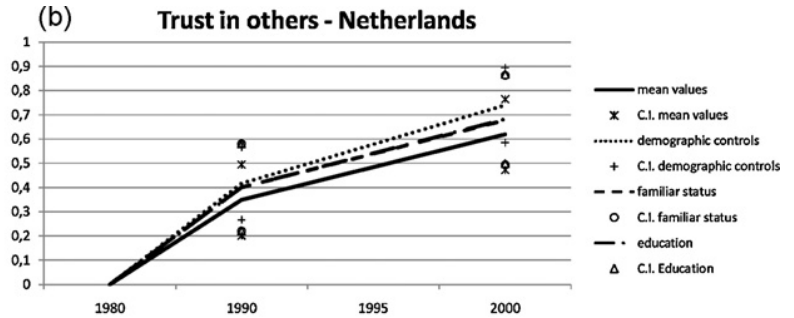

Fig. B.9. Trends about membership in Putnam's groups (a), trust in others (b) and unpaid putnamian voluntary work (c) for the Netherlands from 1980 to 2000.
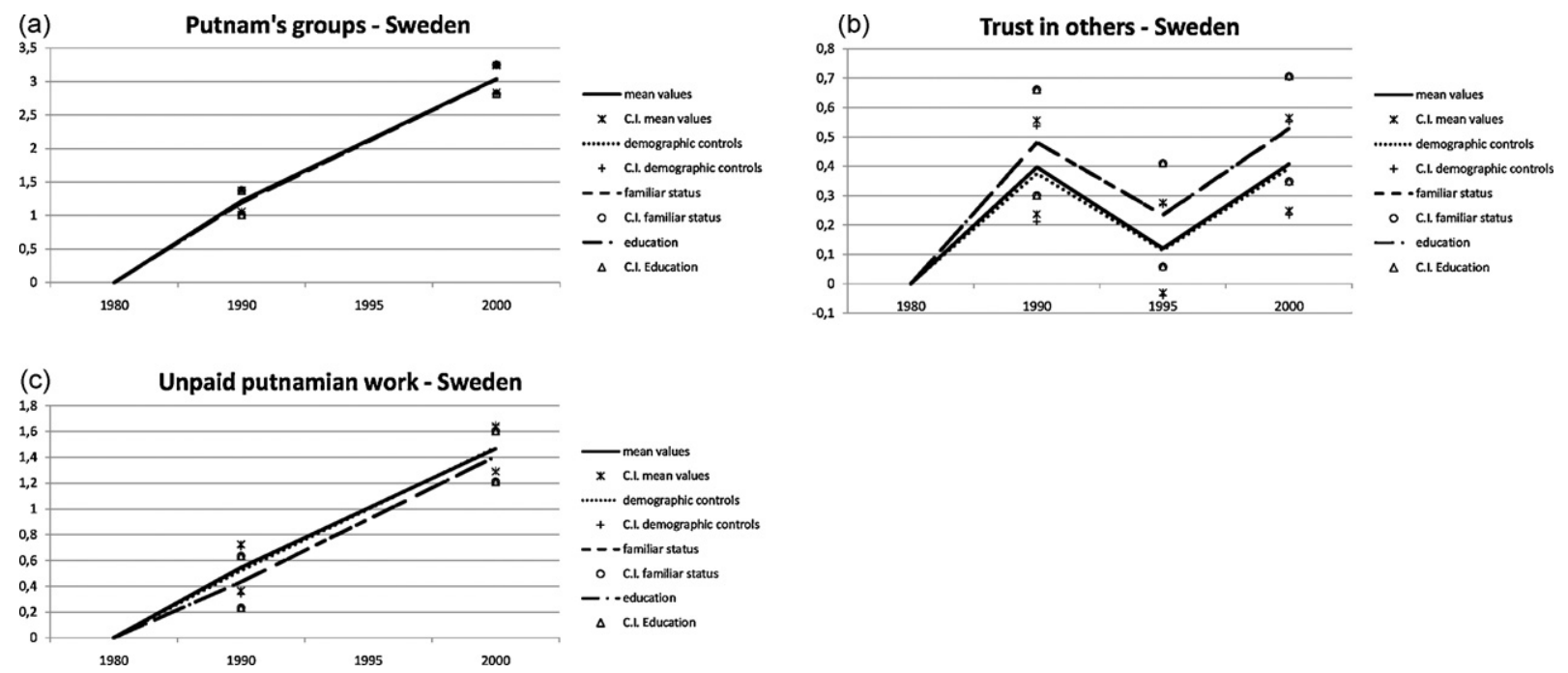

Fig. B.10. Trends about membership in Putnam's groups (a), trust in others (b) and unpaid putnamian voluntary work (c) for Sweden from 1980 to 2000. 
(a)

Putnam's groups - France

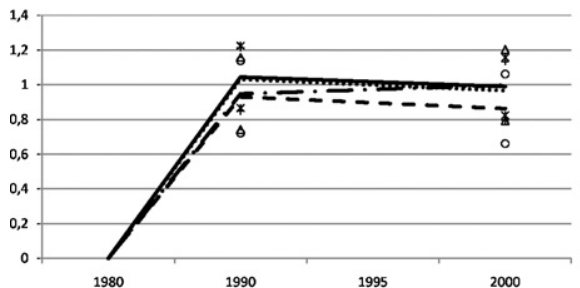

- mean values ........ demographic controls + C.I demographic controls - - - familiar status ○ c.l familiar status - eduction

$1980-1990 \quad 1995$

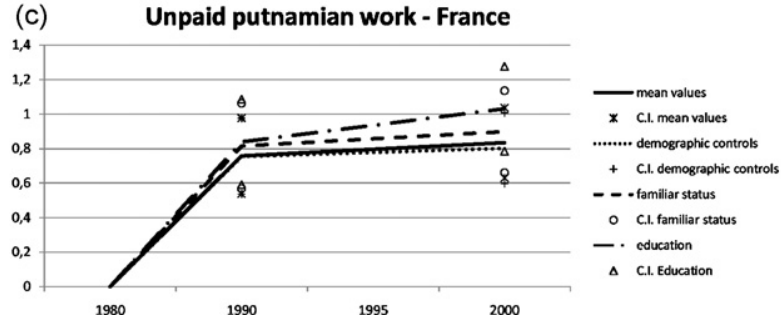

(b) Trust in others - France

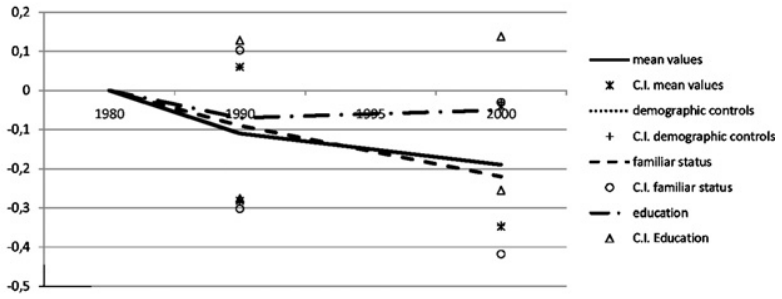

Fig. B.11. Trends about membership in Putnam's groups (a), trust in others (b) and unpaid putnamian voluntary work (c) for France from 1980 to 2000.

(a)

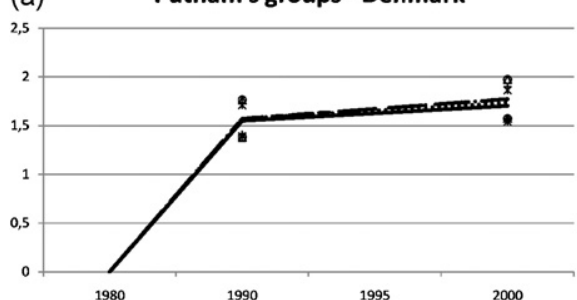

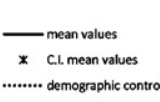

demographic controls

- - familiar status

- c.l familiar status

- education

$\Delta$ C.. Education

\section{(b) Trust in others - Denmark}

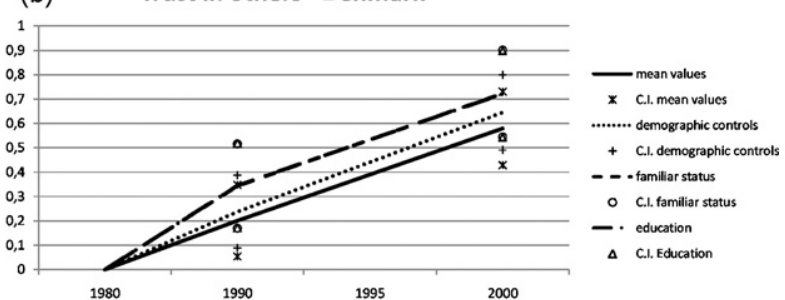

(c) Unpaid putnamian work - Denmark

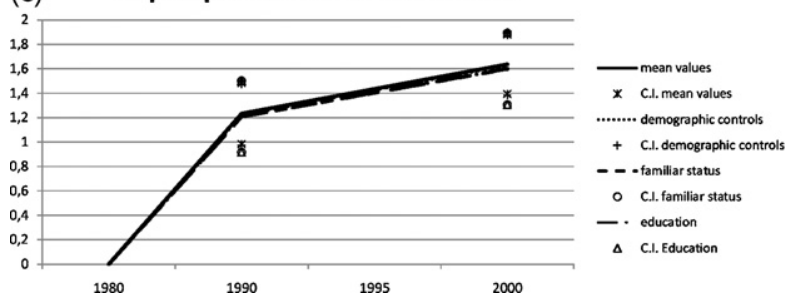

Fig. B.12. Trends about membership in Putnam's groups (a), trust in others (b) and unpaid putnamian voluntary work (c) for Denmark from 1980 to 2000.

(a) Putnam's groups - Norway

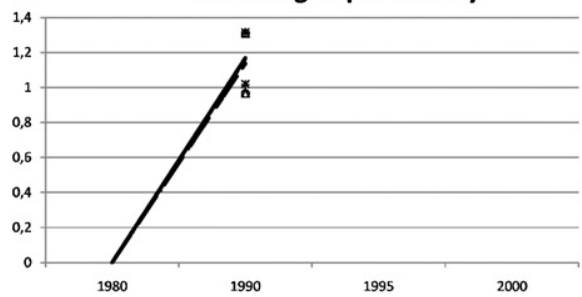

(c) Unpaid putnamian work - Norway

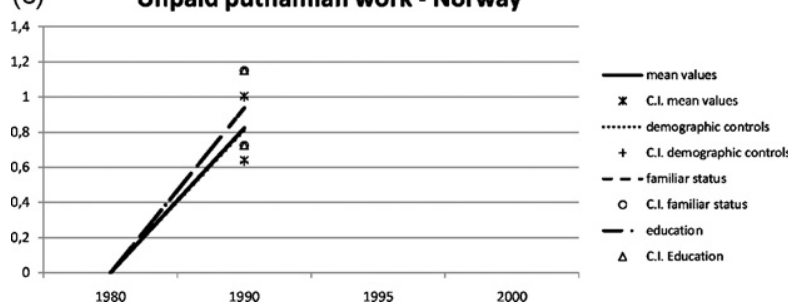

(b) Trust in others - Norway

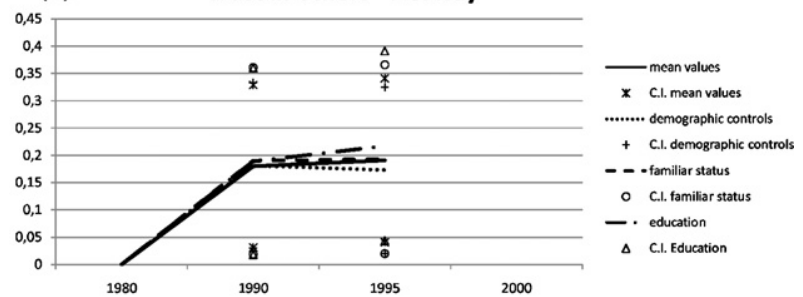

Fig. B.13. Trends about membership in Putnam's groups (a), trust in others (b) and unpaid putnamian voluntary work (c) for Norway from 1980 to 2000. 

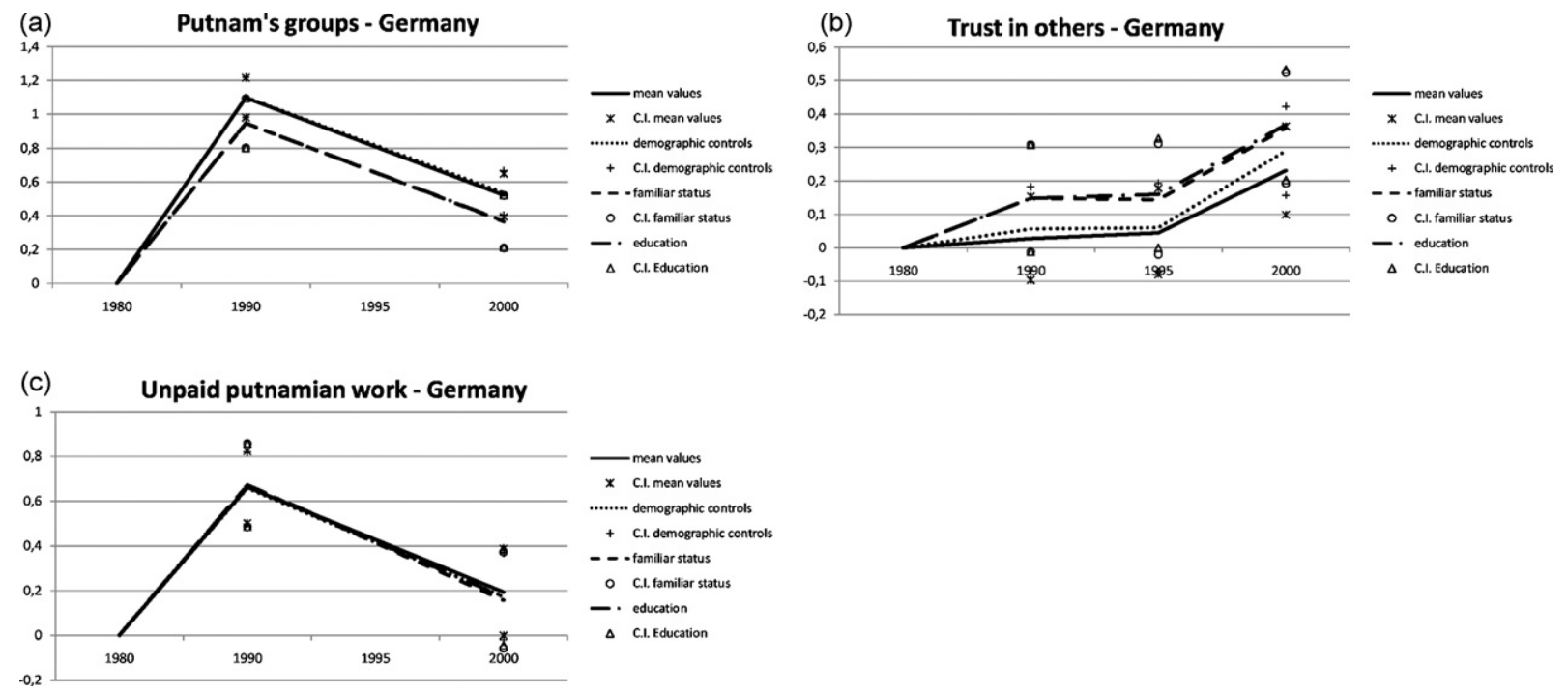

Fig. B.14. Trends about membership in Putnam's groups (a), trust in others (b) and unpaid putnamian voluntary work (c) for Germany from 1980 to 2000.

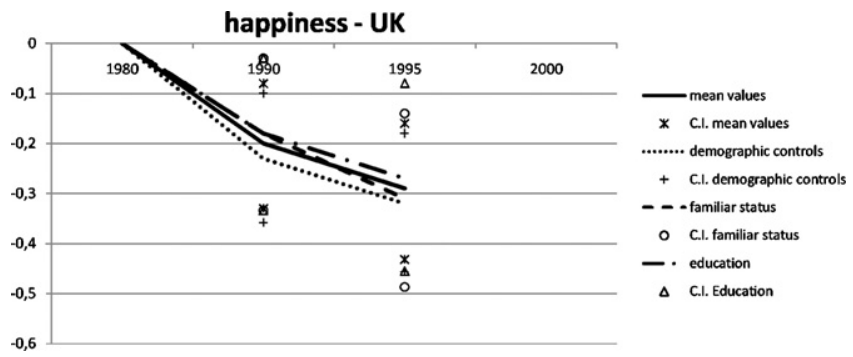

Fig. B.15. Subjective well-being trends for Great Britain from 1980 to 2000.
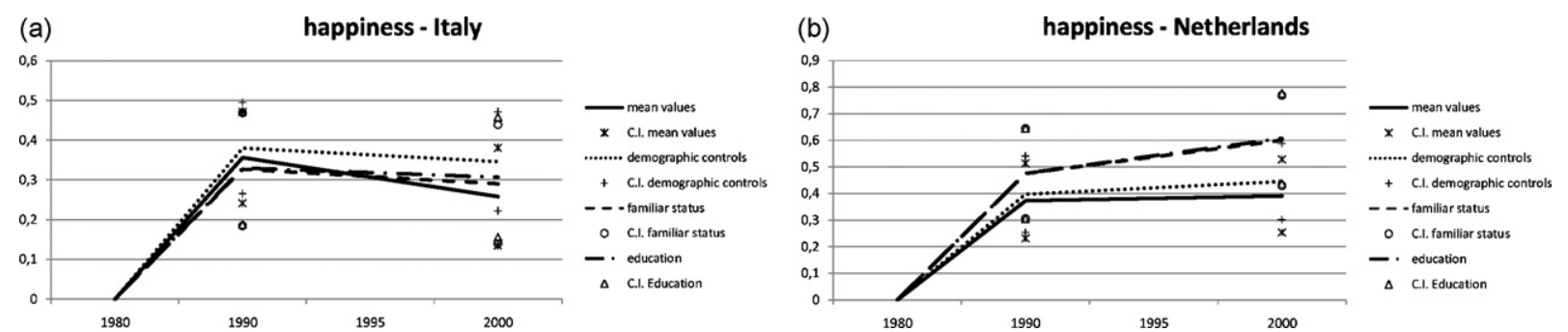

Fig. B.16. Subjective well-being trends for (a) Italy and (b) Netherlands from 1980 to 2000.
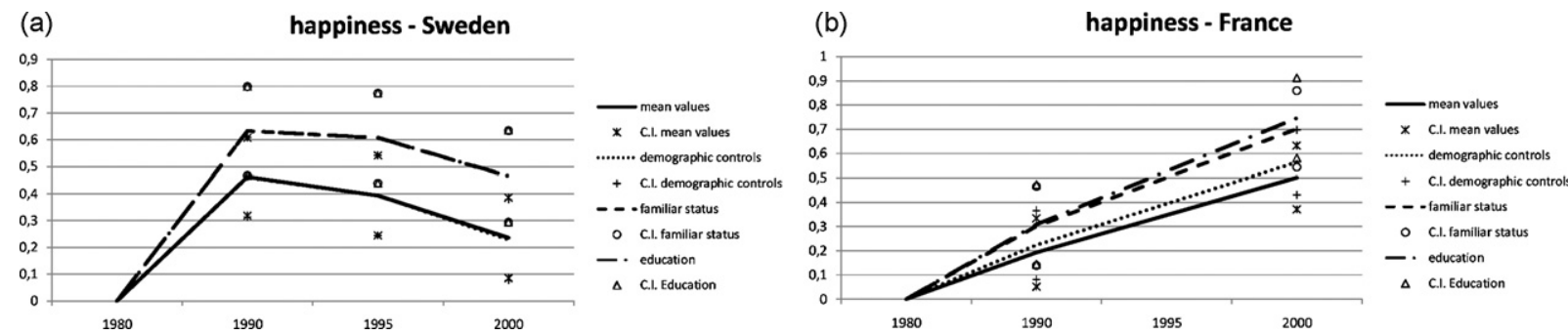

Fig. B.17. Subjective well-being trends for (a) Sweden and (b) France from 1980 to 2000 
(a)

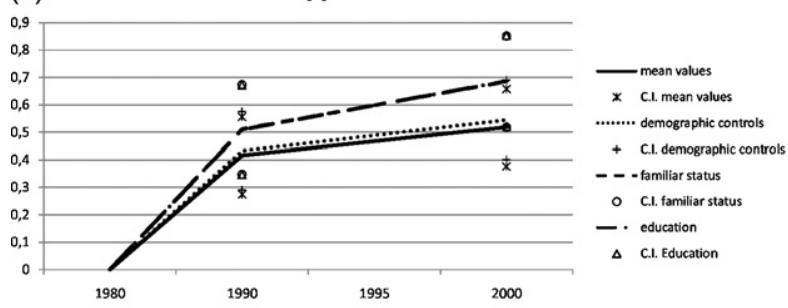

(b)

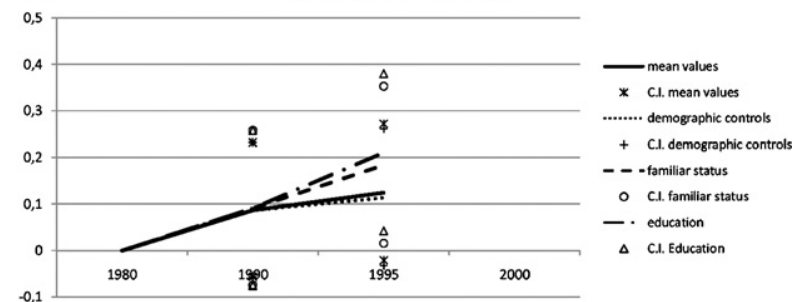

Fig. B.18. Subjective well-being trends for (a) Denmark and (b) Norway from 1980 to 2000.

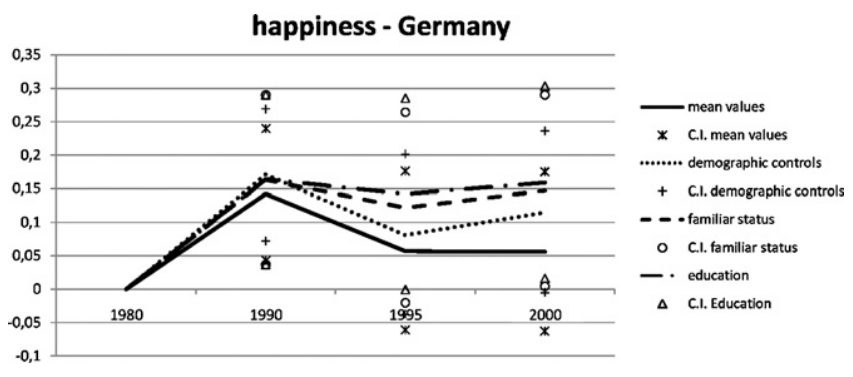

Fig. B.19. Subjective well-being trends for Germany from 1980 to 2000

\section{References}

Adam, F., 2008. Mapping social capital across Europe: ndings, trends and methodological shortcomings of cross-national surveys. Social Science Information 47 (2), 159-186.

Aguiar, M., Hurst, E., 2006. Measuring trends in leisure: the allocation of time over five decades. Federal Reserve Bank of Boston, Working Papers no. 2.

Arts, W., Halman, L., 2004. European Values at the Turn of the Millennium. Brill NV, Leiden, the Netherlands.

Bartolini, S., Bilancini, E., Pugno, M., 2008. American Declines in Social Capital and Happiness: Is There Any Linkage? Siena, Mimeo.

Bartolini, S., Bonatti, L., 2003. Endogenous growth and negative externalities. Journal of Economics, 79

Blanchower, D.G., Oswald, A.J., 2004. Well-being over time in Britain and the USA. Journal of Public Economics, 88.

Bruni, L., Stanca, L., 2006. Watching alone: relational goods, television and happiness. Journal of Economic Behavior and Organization.

Costa, D.L., Kahn, M.E., 2003. Understanding the decline in social capital, 1952-1998. Kyklos 56, 17-46.

Deci, E.L., Ryan, R.M., 1985. Intrinsic Motivation and Self-determination in Human Behaviour. Plenum Press, New York.

Delhey, J., Newton, K., 2005. Predicting cross-national levels of social trust: global pattern or nordic exceptionalism? European Sociological Review 21, 311-327.

Durlauf, S.N., 2002. On the empirics of social capital. The Economic Journal 112 (November (483)).

Durlauf, S.N., Fafchamps, M., 2004. Social capital. The Centre for the Study of African Economies Working Paper Series (214).

Easterlin, R.A., 1974. Does economic growth improve the human lot? Some empirical evidence. In: David, P.A., Melvin, W.R. (Eds.), Nations and Households in Economic Growth. Stanford University Press, CA, pp. 98-125.

Helliwell, J.F., 2001. Social capital, the economy and wellbeing, The Review of Economic Performance: The Longest Decade: Canada in the 1990s. Centre for the Study of Living Standards, Ottawa, Canada.

Helliwell, J.F., 2002. How's life? Combining individual and national variables to explain subjective well-being. National Bureau of Economic Research Working Paper series (July 9065)

Helliwell, J.F., 2006. Well-being, social capital and public policy: what's new? The Economic Journal 116 (March).
Knack, S., 2003. Groups, growth and trust: cross-country evidence on the olson and putnam hypotheses. Public Choice (117), 341-355.

Ladd, E., 2003. The data just don't show erosion of America's social capital. Public Perspective 7, 1-30.

Leigh, A., 2003. Entry on "Trends in Social Capital", Christensen, K., Levinson, D. (Eds.), Encyclopedia of Community: From the Village to the Virtual World. Sage, Thousand Oaks, CA.

Morales, L. Changing patterns of associational involvement in Europe. Workshop 8 -The changing structure of civil society, directed by Derrick Purdue \& Mario Diani, ECPR Joint Sessions, Uppsala, 2004

Norris, P., 2004. Making democracies work: social capital and civic engagement in 47 societies. British Journal of Political Science 35, 149-167.

OECD, 2001a. Are trust and civic engagement declining in OECD countries? In the Well-Being of Nations: The Role if Human and Social Capital. OECD, Paris, pp. 99-103.

OECD, 2001b. The Evidence on Social Capital. The Well-Being of Nations: The Role if Human and Social Capital. OECD, Paris, pp. 39-63.

Olson, M., 1982. The Rise and Decline of Nations: Economic Growth, Stagflation and Social Rigidities. Yale University Press, New Haven.

Paxton, P., 1999. Is social capital declining in the United States? a multiple indicator assessment. American Journal of Sociology 105 (July (1)), 88-127.

Putnam, R.D., 1993. Making Democracy Work: Civic Traditions in Modern Italy. Princeton University Press, Princeton, NJ.

Putnam, R., 2000. Bowling Alone. Simon \& Schuster, New York.

Putnam, R., 2001. Social capital measurement and consequences. Isuma 2 (Spring (1)).

Putnam, R., 2002. Democracy in Flux, the Evolution of Social Capital in Contemporary Society. Oxford University Press, Oxford.

Robinson, R.V., Jackson, E.F., 2001. Is trust in others declining in America? An ageperiod-cohort analysis. Social Science Research 30, 117-147.

Sarracino, F., 2008. Subjective well-being in Low Income Countries: positional, relational and social capital components. Studi e Note di economia, Anno XIII, n. 3, pp. 449-477.

Schuller, T., Baron, S., Field, J., 2000. Social capital: a review and critique. In: Schuller, T., Baron, S., Field, J. (Eds.), Social Capital: Critical Perspectives. Oxford University Press, pp. 1-39.

Stevenson, B., Wolfers, J., 2008. Economic growth and subjective well-being: reassessing the Easterlin paradox. IZA DP (August (3654)).

Stolle, D., Hooghe, M., 2004. Inaccurate, exceptional, one-sided or irrelevant? The debate about the alleged decline of social capital and civic engagement in Western societies. British Journal of Political Science, 149-167.

Tinggaard Svendsen, G., Haase, G.L., Svendsen, 2009. Handbook of Social Capital: The Troika of Sociology, Political Science and Economics. Edward Elgar Publishing Limited, Cheltenham, UK.

Van Deth, J.W., Castiglione, D., Wolleb, G., 2008. Handbook of Social Capital. Oxford University Press, New York, USA

Van OOrschot, W., Arts, W., Gelissen, J., 2006. Social capital in Europe, measurement and social and regional distribution of a multifaceted phenomenon. Acta Sociologica 49 (June (2)), 149-167.

Van Schaik, T., 2002. Social capital in the European Values Study surveys. In: Country Paper Prepared for the OECD-ONS International Conference on Social Capital Measurement London, September, pp. 25-27. 